San Jose State University

SJSU ScholarWorks

Master's Theses

Master's Theses and Graduate Research

Summer 2012

\title{
Synthesis and Evaluation of Four Novel Stationary Phases in High Pressure Liquid Chromatography
}

Niloofar Salehi

San Jose State University

Follow this and additional works at: https://scholarworks.sjsu.edu/etd_theses

\section{Recommended Citation}

Salehi, Niloofar, "Synthesis and Evaluation of Four Novel Stationary Phases in High Pressure Liquid Chromatography" (2012). Master's Theses. 4209.

DOI: https://doi.org/10.31979/etd.x6fh-zd4k

https://scholarworks.sjsu.edu/etd_theses/4209

This Thesis is brought to you for free and open access by the Master's Theses and Graduate Research at SJSU ScholarWorks. It has been accepted for inclusion in Master's Theses by an authorized administrator of SJSU ScholarWorks. For more information, please contact scholarworks@sjsu.edu. 


\author{
A Thesis \\ Presented to \\ The Faculty of the Department of Chemistry \\ San José State University
}

\author{
In Partial Fulfillment \\ of the Requirements for the Degree \\ Master of Science
}

by

Niloofar Salehi

August 2012 
(C) 2012

Niloofar Salehi

ALL RIGHTS RESERVED 
The Designated Thesis Committee Approves the Thesis Titled

SYNTHESIS AND EVALUATION OF FOUR NOVEL STATIONARY PHASES IN HIGH PRESSURE LIQUID CHROMATOGRAPHY

by

Niloofar Salehi

APPROVED FOR THE DEPARTMENT OF CHEMISTRY

SAN JOSÉ STATE UNIVERSITY

August 2012

Dr. Joseph J. Pesek

Department of Chemistry

Dr. Daryl K. Eggers

Department of Chemistry

Dr. Roger H. Terrill

Department of Chemistry 


\section{ABSTRACT \\ SYNTHESIS AND EVALUATION OF FOUR NOVEL STATIONARY PHASES IN HPLC}

by Niloofar Salehi

High pressure liquid chromatography is one of the most frequently used methods for the separation of mixtures. Chemically modified silica hydride stationary phases, due to their unique selectivity and versatility, have been the focus of an increasing number of studies in the past several years. The objective of fabricating stationary phases with dual properties is to achieve separation of both polar and non-polar components in a single analysis. Due to an increasing demand in the fields of drug discovery, proteomics, and metabolomics to analyze numerous samples with a wide range of polarities, there is a need for more versatile stationary phases for a wide range of applications. In order to meet the above objective, in this research four novel silica hydride-based columns were synthesized using a hydrosilation procedure. The characterization of each column was

done using a series of polar and nonpolar compounds by studying their aqueous normal phase and reversed-phase chromatographic behavior. Under these conditions, it turned out that two columns showed both RP and ANP behaviors. Of the remaining columns, one exhibited ANP behavior only and the other performed only in the reversed-phase mode. 


\section{ACKNOWLEDGMENTS}

I would like to offer my sincere gratitude to everyone who supported me along the way. My special thanks to Professor Joseph Pesek and Dr. Maria Matyska for their guidance and being the compass of my learning. I am immensely thankful to Professor Pesek for his valuable advices and his willingness to share his knowledge with me and to Dr. Matyska for her availability to teach me patiently. I am beyond words to show my appreciation to Dr. Daryl Eggers for all his assistance every step of the way and Dr. Roger Terrill for his words of encouragement. I thank you very much for giving me the honor of being my committee members. I also would like to thank Dr. Bradley Stone for all his support and understandings.

I would like to make a special dedication to my loving family for supporting me through this journey. To my patient parents, Molook and Mehdi, thank you for believing in me. I am here today because of you. I would like to thank Nader and Shadi for waiting patiently to witness this day. Lastly, I would like to extend my eternal thanks to Faranak and Taraneh for their continued support. 


\section{TABLE OF CONTENTS}

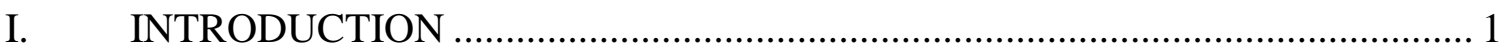

A. Historical Background ........................................................................ 1

B. High Pressure Liquid Chromatography (HPLC) ......................................... 2

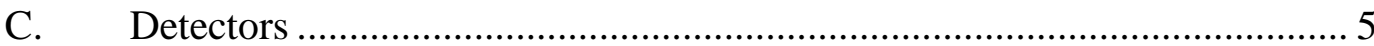

1. The Ultraviolet (UV) detector......................................................... 5

2. Diode array detector...................................................................... 5

D. Modes of Separation ............................................................................... 6

1. Normal phase high performance liquid chromatography ................ 6

2. $\quad$ Reversed phase high performance liquid chromatography.............. 6

3. Aqueous normal phase high performance liquid chromatography. 7

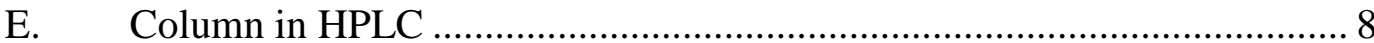

1. Column efficiency ......................................................................... 8

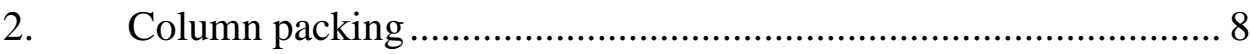

3. $\quad$ Methods to modify silica surface ...................................................... 10

i. Esterification .................................................................... 11

ii. Organosilanization ........................................................... 12

iii. Grignard reaction .......................................................... 13

iv. Silanization/ Hydrosilation .................................................. 13

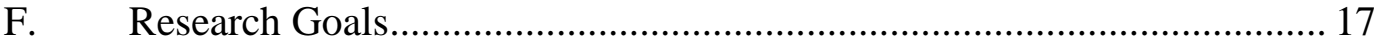




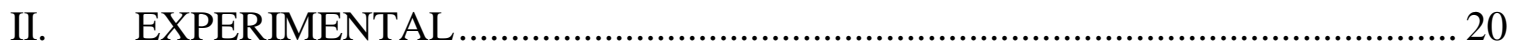

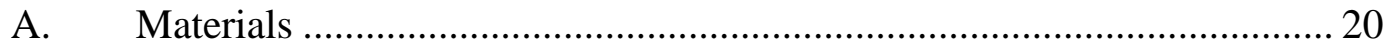

1. Silanization: Preparation of silanol ................................................ 20

2. Hydrosilation: Preparation of stationary phase............................... 21

3. $\quad$ HPLC: Mobile phase preparation ……………………………....... 22

4. HPLC analysis: Compounds analyzed with the stationary phases 23

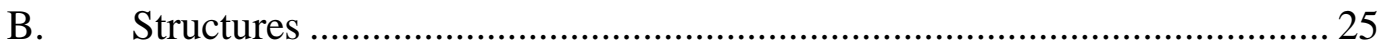

1. Organic compounds used in the synthesis of the bonded

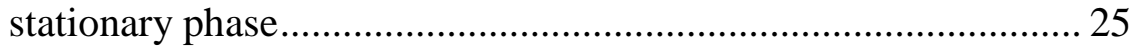

2. Analytes used for the HPLC evaluations ....................................... 26

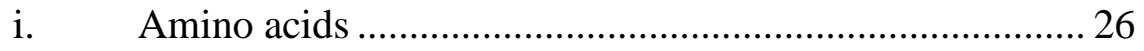

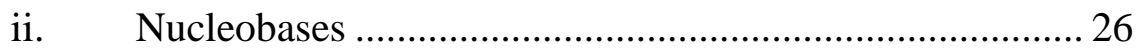

iii. Neurotransmitters........................................................... 27

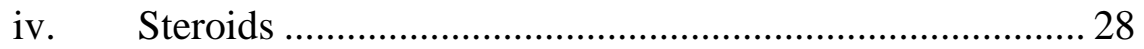

v. Polycyclic aromatic hydrocarbons ...................................... 29

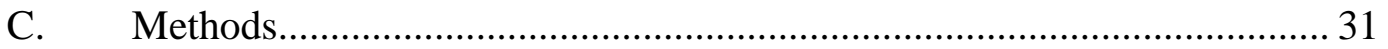

1. Silanization: Synthesis of silica hydride ........................................ 31

2. Hydrosilation: Synthesis of stationary bonded phase ..................... 32

i. $\quad$ Preparation of 1,8-nonadiyne bonded phase ....................... 32

ii. $\quad$ Prepareation of $m$-diethynylbenzene bonded phase........... 34

iii. $\quad$ Preparation of 5-cyano-1-pentyne bonded phase............... 35

iv. Preparation of 6-chloro-1-hexyne bonded phase ............... 35 
v. Preparation of 4-ethynylanaline bonded phase ................ 36

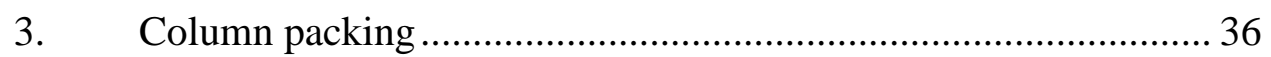

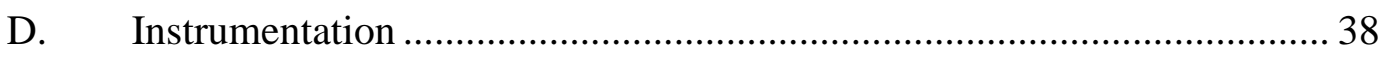

1. Diffuse reflectance infrared fourier transform (DRIFT)

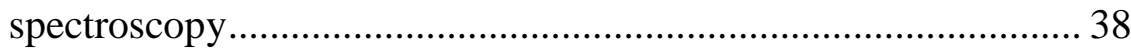

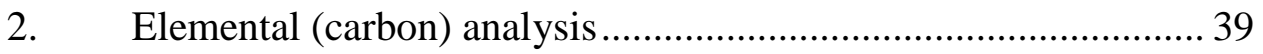

3. Nuclear magnetic resonance (NMR) spectroscopy..................... 39

4. High performance liquid chromatography (HPLC).................... 41

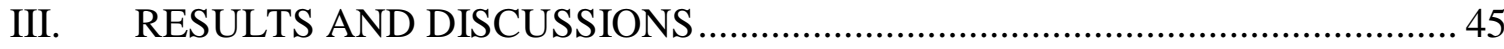

A. DRIFT Spectroscopic Characterization .......................................... 45

1. Silica hydride characterization............................................ 45

2. Moiety surface characterization............................................ 45

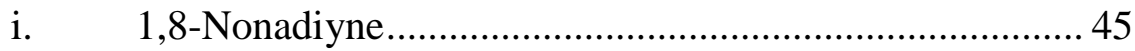

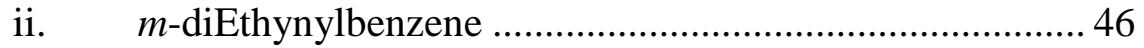

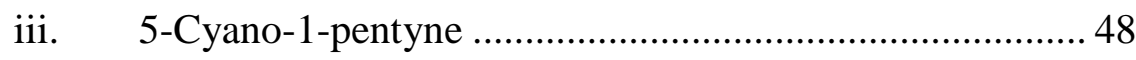

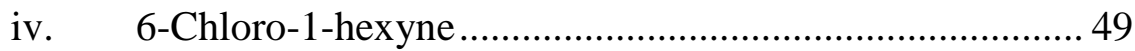

v. 4-Ethynylanaline ..................................................... 50

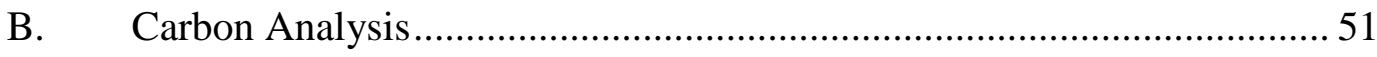

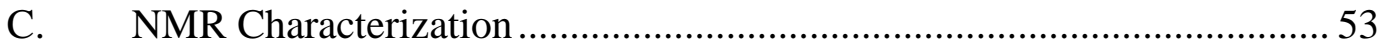

1. Solid state NMR (SSNMR): Characterization with ${ }^{29} \mathrm{Si}$ CP/MAS

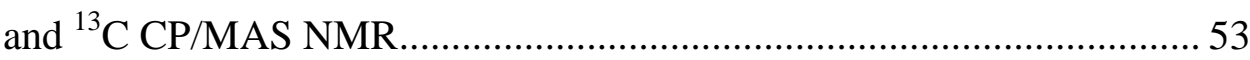

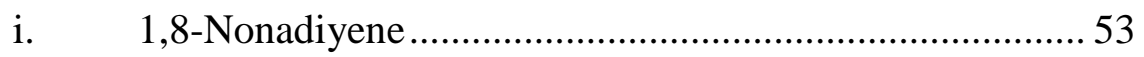


ii. $\quad$ 5-Cyano-1-pentyne ................................................. 55

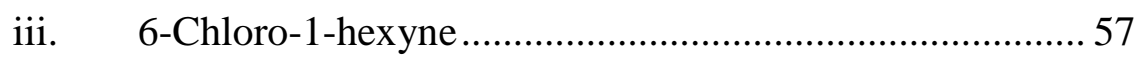

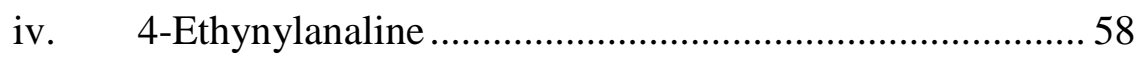

D. HPLC Chromatographic Evaluation of the Columns ............................. 60

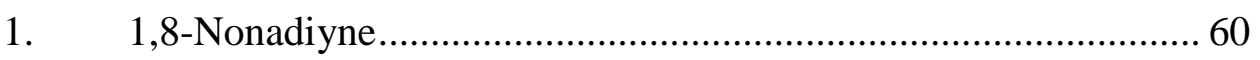

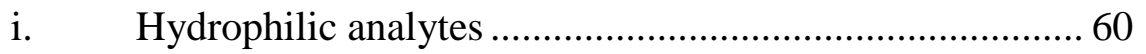

a. Reproducibility study (\%RSD) ........................... 66

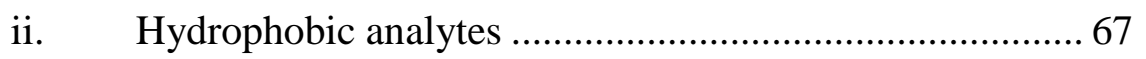

iii. Addition of formic acid (buffer) ...................................... 72

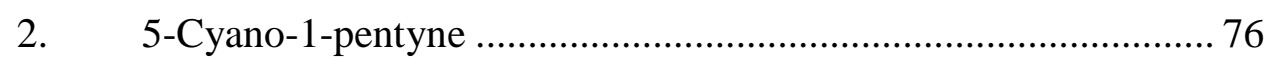

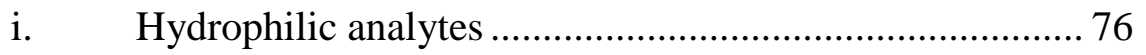

ii. Hydrophobic analytes ............................................... 81

iii. Addition of formic acid (buffer) .................................. 87

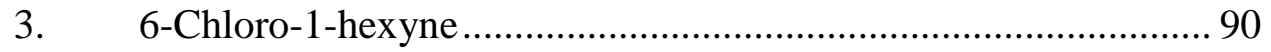

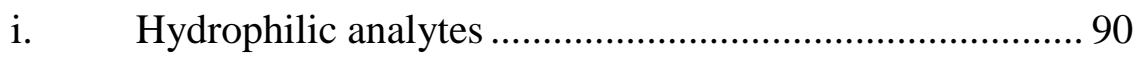

ii. Hydrophobic analytes ............................................... 94

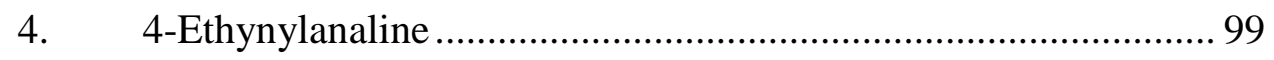

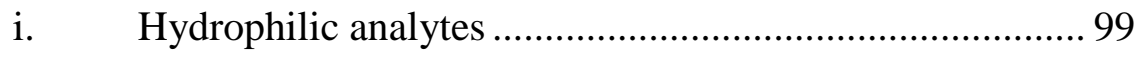

ii. Hydrophobic analytes ............................................. 103

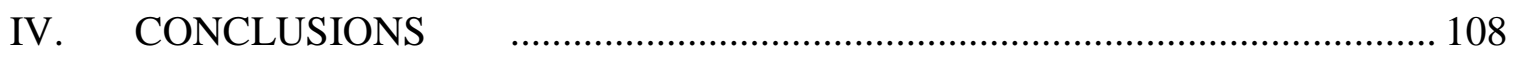

REFERENCES 


\section{LIST OF FIGURES}

Figure 1. Schematic view of basic components in HPLC ...................................... 3

Figure 2. Different types of silanols on the silica surface .................................. 9

Figure 3. Type B silica and strong water molecule association through hydrogen ....

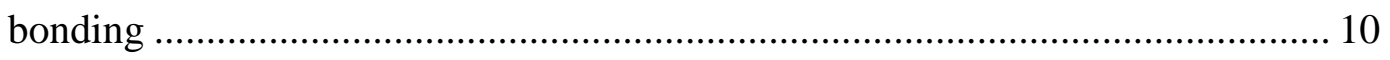

Figure 4. Type C silica and elimination of water molecule ................................. 11

Figure 5. Schematic comparison of Type-B vs. Type-C silica ….......................... 15

Figure 6. Ideal RP and ANP regions retention map on Si-H stationary phase........ 19

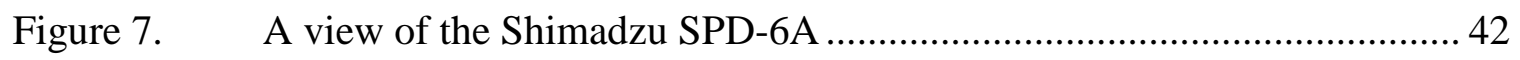

Figure 8. A view of the HP 1090 HPLC system .............................................. 44

Figure 9. DRIFT spectrum for Silica hydride identification ................................ 46

Figure 10. DRIFT spectrum of 1,8-nonadiyne bonded phase ............................... 47

Figure 11. DRIFT spectrum for $m$-diethynylbenzene bonded phase ........................ 48

Figure 12. DRIFT spectrum of 5-cyano-1-pentyne bonded phase ........................... 49

Figure 13. DRIFT spectrum of 6-chloro-1-hexyne bonded phase ........................... 50

Figure 14. DRIFT spectrum of 4-ethynylanaline bonded phase ............................. 51

Figure 15. $\quad{ }^{29}$ Si CP/ MAS NMR spectrum for the 1,8-nonadiyne stationary phase.... 54

Figure 16. $\quad{ }^{13} \mathrm{C} \mathrm{CP/} \mathrm{MAS} \mathrm{NMR} \mathrm{spectrum} \mathrm{for} \mathrm{the} \mathrm{1,8-nonadiyne} \mathrm{stationary} \mathrm{phase} \mathrm{....} 54$

Figure 17. $\quad{ }^{29} \mathrm{Si} \mathrm{CP} / \mathrm{MAS}$ NMR spectrum for the 5-cyano-1-pentyne stationary

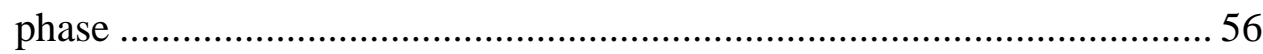


Figure 18. $\quad{ }^{13} \mathrm{C}$ CP/ MAS NMR spectrum for the 5-cyano-1-pentyne stationary

phase 56

Figure 19. $\quad{ }^{29} \mathrm{Si} \mathrm{CP} / \mathrm{MAS}$ NMR spectrum for the 6-chloro-1-hexyne stationary

phase 57

Figure 20. $\quad{ }^{13} \mathrm{C} \mathrm{CP/} \mathrm{MAS} \mathrm{NMR} \mathrm{spectrum} \mathrm{for} \mathrm{the} \mathrm{6-chloro-1-hexyne} \mathrm{stationary}$

phase 58

Figure 21. $\quad{ }^{29} \mathrm{Si} \mathrm{CP} / \mathrm{MAS}$ NMR spectrum for the 4-ethynylanaline stationary

phase

Figure 22. $\quad{ }^{13} \mathrm{C} \mathrm{CP/} \mathrm{MAS} \mathrm{NMR} \mathrm{spectrum} \mathrm{for} \mathrm{the} \mathrm{4-ethynylanaline} \mathrm{stationary}$

phase 59

Figure 23. Retention map of the amino acids, phenylalanine and phenylglycine, using the 1,8-nonadiyne column 63

Figure 24. Retention map of the nucleobases, adenine and guanine, using the 1,8-nonadiyne column...... 65

Figure 25. Retention map of the steroids, adrenosteron, $\Delta^{4}$-androsten-3,17-dione, and corticosterone using 1,8-nonadiyne column 69

Figure 26. Retention map of adenine, dopamine, and $\Delta^{4}$-androsten-3,17-dione, using 1,8-nonadiyne column 71

Figure 27. Retention map of the polar and non-polar analytes using the 1,8-nonadiyne column...... 72

Figure 28. Retention map of two polar analytes using the 1,8-nonadiyne column and $0.1 \% \mathrm{FA}$ in the mobile phase 73 
Figure 29. Retention map of two polar analytes using the 1,8-nonadiyne column without buffer.

Figure 30. Retention map of the polar analytes using the 1,8-nonadiyne column and $0.1 \%$ FA in the mobile phase 74

Figure 31. Retention map of the polar nucleobases using the 5-cyano-1-pentyne

column. 79

Figure 32. Retention map of the polar neurotransmitters using the 5-cyano-1-

pentyne column. 81

Figure 33. Retention map of the non-polar steroids using the 5-cyano-1-pentyne

column

Figure 34. Retention map of the non-polar analytes using the 5-cyano-1-pentyne

column. 85

Figure 35. Retention map of the polar and non-polar analytes using the 5-cyano-1pentyne column. 86

Figure 36. Retention map of a polar and non-polar analytes using the 5-cyano-1pentyne column and $0.1 \mathrm{FA}$ in the mobile phase 89

Figure 37. Retention map of a polar and non-polar analytes using the 5-cyano-1-

pentyne column without buffer 89

Figure 38. Retention map of the polar amino acids using the 6-chloro-1-hexyne

column. 91

Figure 39. Retention map of the polar nucleobases using the 6-chloro-1-hexyne column 
Figure 40. Retention map of the steroid analytes using the 6-chloro-1-hexyne

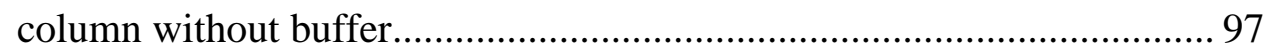

Figure 41. Retention map of the polycyclic aromatic hydrocarbon analytes

using the 6-chloro-1-hexyne column without buffer .................................. 98

Figure 42. Retention map of the polar and non-polar analytes using the 6-chloro-1-

hexyne column without buffer ................................................................ 99

Figure 43. Retention map of the polar analytes using the 4-ethynylalanine

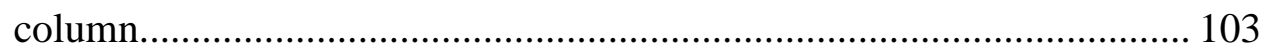

Figure 44. Retention map of the hydrophobic analytes using the 4-ethynylalanine

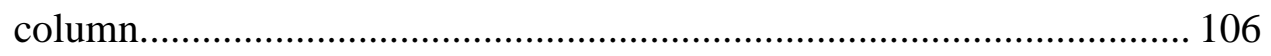

Figure 45. Retention map of the polar and non-polar analytes using the

4-ethynylanaline column without buffer................................................ 107 


\section{LIST OF TABLES}

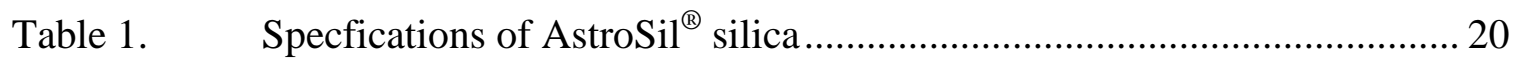

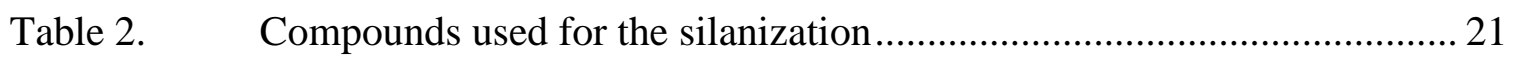

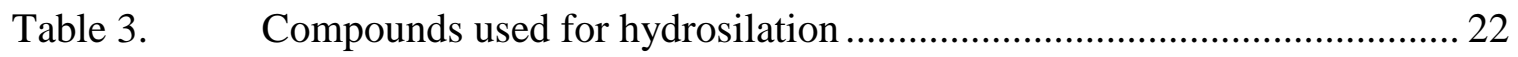

Table 4. Compounds used in the mobile phase.............................................. 23

Table 5. Amino acids (hydrophilic/ polar) ........................................................ 23

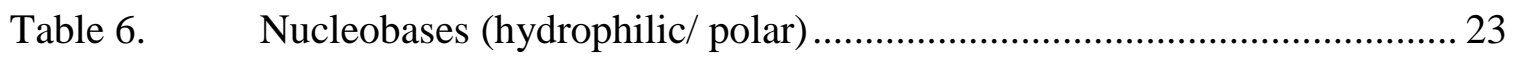

Table $7 . \quad$ Neurotransmitters (hydrophilic/ polar) .............................................. 24

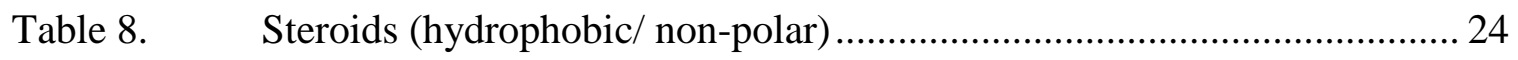

Table 9. Polycyclic aromatic hydrocabons (PAH) (hydrophobic/ non-polar) ........ 24

Table 10. Carbon analysis of the newly synthesized bonded phases....................... 52

Table 11. Retention times of the hydrophilic amino acids using the 1,8-nonadiyne

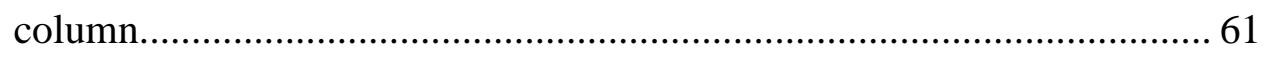

Table 12. Retention times of the hydrophilic nucleobases using the1,8-nonadiyne

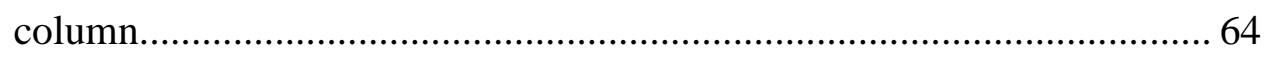

Table 13. Retention times of the hydrophilic neurotransmitters using the 1,8-

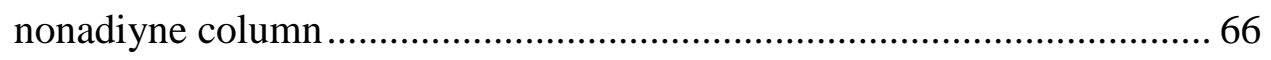

Table 14. Reproducibility for 10 injections of two amino acids using the 1,8nonadiyne bonded stationary phase

Table 15. Retention times of the hydrophobic steroids using the 1,8-nonadiyne

column. 68 
Table 16. Retention times of the hydrophobic polycyclic aromatic hydrocarbons using the 1,8-nonadiyne column 70

Table 17. Retention times of the hydrophilic analytes using the 1,8-nonadiyne column with $0.1 \%$ formic acid buffer in the mobile phase. 75

Table 18. Retention times of the hydrophilic amino acids using the 5-cyano-1-

pentyne column 77

Table 19. Retention times of the hydrophilic nucleobases using the 5-cyano-1pentyne column. 78

Table 20. Retention times of the hydrophilic neurotransmitters using the 5-cyano-1-pentyne column 80

Table 21. Retention times of the hydrophobic steroids using the 5-cyano-1pentyne column 82

Table 22. Retention times of the hydrophobic polycyclic aromatic hydrocarbons using the 5-cyano-1-pentyne column

Table 23. Retention times of epinephrine and pyrene using the 5-cyano-1-pentyne column with $0.1 \%$ formic acid buffer in the mobile phase. 88

Table 24. Retention times of the hydrophilic amino acids using the 6-chloro-1hexyne column 90

Table 25. Retention times of the hydrophilic nuclueobases using the 6-chloro-1hexyne column 92

Table 26. Retention times of the hydrophilic neurotransmitters using the 6-chloro-1-hexyne column. 
Table 27. Retention times of the hydrophobic steroids using the 6-chloro-1-hexyne

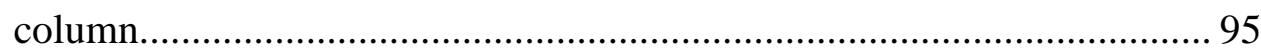

Table 28. Retention times of the hydrophobic polycyclic aromatic hydrocarbons

using the 6-chloro-1-hexyne column ......................................................... 96

Table 29. Retention times of the hydrophilic amino acids using the 4-ethynylanaline

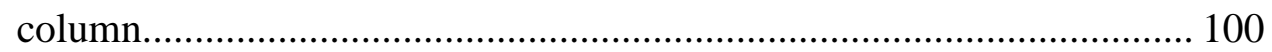

Table 30. Retention times of the hydrophilic nuclueobases using the

4-ethynylanaline column......................................................................... 101

Table 31. Retention times of the hydrophilic neurotransmitters using the

4-ethynylanaline column.......................................................................... 102

Table 32. Retention times of the hydrophobic steroids using 4-ethynylanaline

column

Table 33. Retention times of the hydrophobic polycyclic aromatic hydrocarbons

using the 4-ethynylanaline column ......................................................... 105 


\section{INTRODUCTION}

\section{A. Historical Background}

Chromatography is an essential separation technique by which components of a mixture are separated based on their physical and chemical properties. This technique has numerous applications (e.g., separation, purification, and characterization) in various fields such as pharmaceutical, biomedical, industrial, environmental, and more. The technique takes advantage of the physical properties of components and their interactions and affinities with the two different phases available in the system: mobile and stationary phases. The solutes with polarities similar to the polarity of the mobile phase have higher affinity for that phase and vice versa. These solutes tend to leave or elute with the mobile phase, and those that tend to stay behind and interact with the stationary phase elute later. Other factors that could influence these interactions include (but not limited to) the size of the particles, the charge or presence of ions (for ionic solutes), and the various combinations [1]. The analyte's traveling time through the stationary phase is called the retention time. Retention time is characteristic and unique to each compound.

Chromatography exists in various formats. The variety is based on the physical state of the mobile phase, the type of the stationary phase, and the separation method. Separation mechanisms such as size exclusion, adsorption, ion exchange, and affinity have resulted in a variety of chromatographic methods. Historically, chromatography started with paper chromatography (PC) and column chromatography (CC). Further developments led to thin layer chromatography (TLC), followed by gas chromatography (GC), and liquid chromatography (LC) [2]. The terms liquid and gas chromatography 
indicate the physical state of the mobile phase and hence the corresponding chromatographic names. Gas chromatography is more suitable for solutes that are volatile but non-decomposable when heated, whereas in liquid chromatography solutes are dissolved in a solvent and a liquid mobile phase carries them along the stationary phase [1]. The early reports on liquid chromatography date back to 1903 with Tswett, an Italian scientist working in a Russian laboratory. He discovered the separation of green pigments of a plant while working with a column of powdered chalk [3].

\section{B. High Pressure Liquid Chromatography (HPLC)}

Until the mid-1960s, liquid chromatography was a technique with a large column filled with an adsorbent packing material, mainly silica gel or alumina. The separation took place by filtering the liquid through the packed material under gravitational force [3]. However, due to the large scale of the materials and instruments, the analytical results were accompanied by poor resolution and low efficiency. It involved large consumption of solvents, generation of a substantial amount of waste, high cost, and low accuracy and precision in results.

In modern analytical chemistry, accuracy and precision in measurements at very low concentrations and high resolutions are essential [2]. To address these requirements, researchers introduced high pressure liquid chromatography (HPLC). HPLC is an effective tool for rapidly improving the resolution and efficiency of each analysis by running the samples at a very high pressure, up to 6000 psi [4]. In a modern HPLC 
system, there are four major components: solvent delivery, sample delivery, separation, and detection available, as shown in Figure 1.

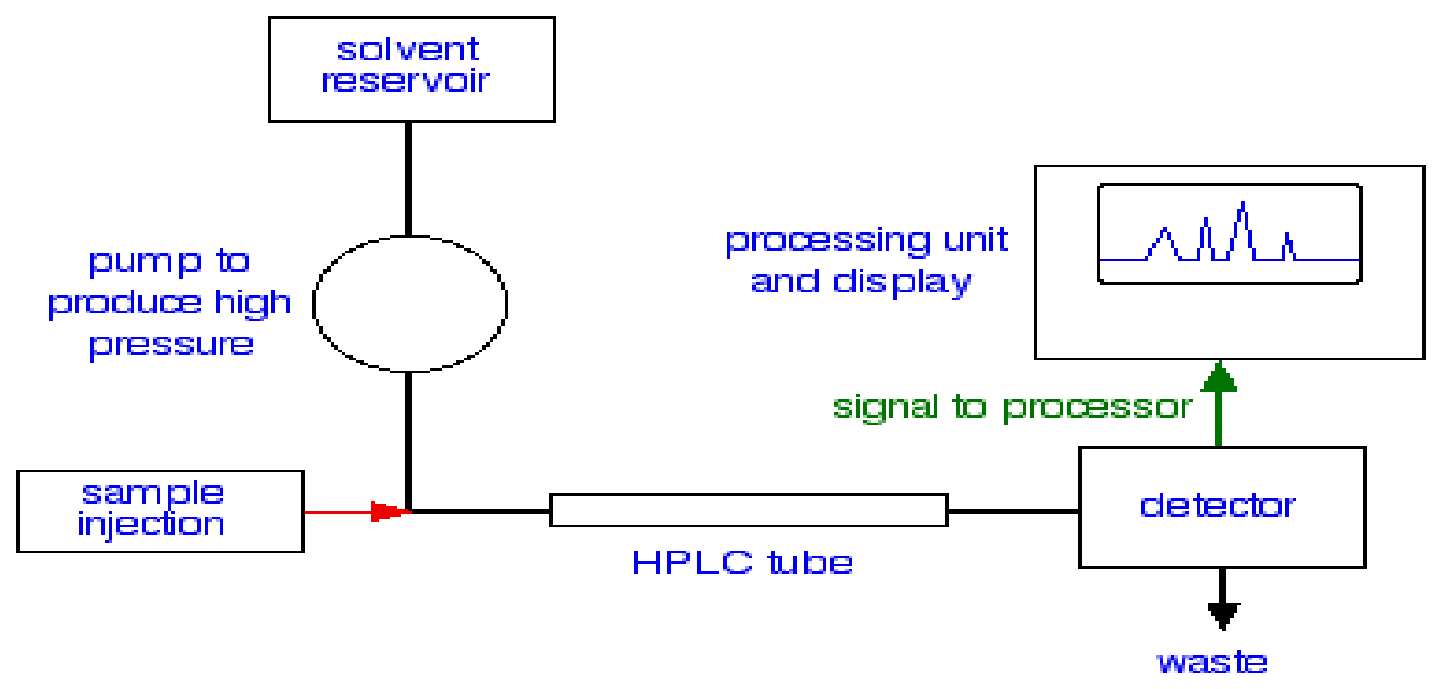

Figure 1. Schematic view of basic components in HPLC

The solvent delivery system is comprised of a pump (the major component), the solvent reservoirs, degassers, mixers, gradient formers, and pulse dampers. The solvent reservoir is specifically beneficial when there is more than one solvent involved in the composition of mobile phase. Generally, solvents have some $\mathrm{O}_{2}$ and $\mathrm{N}_{2}$ dissolved in them, and, if they are not removed, the chromatogram could show them as additional peaks interfering with the analyte data. Pumping Helium gas at a constant flow through a sparger can solve this problem. The gradient system is designed for introducing varying concentrations of the mobile phase per time unit. The mobile phase is pumped through 
the stationary phase (column) at a very high pressure resulting at a flow rate of $0.1-10$ $\mathrm{mL}$ per min [5].

The injector comprises the sample delivery system. There are two ways to introduce the sample, manually or automatically. If the HPLC instrument is equipped with a manual injector, there is a loop that introduces the sample into the system in a continuous flow from a single switch valve. For HPLC instruments with an autosampler, the sample is injected into the column at preset intervals $[1,4,6]$.

Separation takes place in the column or the stationary phase in HPLC. Columns are small stainless steel or polymer tubes with a typical length between 50 and $250 \mathrm{~mm}$ and inner diameter of $4.6 \mathrm{~mm}$, or smaller. The stationary phase is immobilized on small sized porous particles, between 1.5-10 $\mu \mathrm{m}$ [1]. The material for the stationary phase is typically silica or silica modified with organic derivatives.

The detection system in HPLC utilizes a property of the analyte or mobile phase to produce an electronic signal. The signal appears in a form of retention time versus concentration of the analyte in the mobile phase. This output is referred to as the chromatogram. There are a variety of different detectors that exploit different physical and chemical properties of the analytes. Ultraviolet-visible and fluorescence absorbance detectors are able to detect chemicals based on how they absorb or emit these types of light. Mass spectrometers (MS) and refractive index (RI) are other commonly used detectors that respond to a much wider selection of analytes $[1,6]$. 


\section{Detectors}

\section{The Ultraviolet (UV) detector}

Many compounds can absorb UV- visible light in the region of $200-800 \mathrm{~nm}$ (except for compounds without a chromophore), and this is the principle that allows the application of UV detectors. Upon absorption of UV light by the analyte, the intensity of the light that passes to the detectors decreases, and the extent of absorption is directly related to the concentration of the analyte, as described in Beer's law. UV light sources emit wavelengths over a wide range; in simple detectors and with the aid of a filter or monochromator, the wavelength range can be narrowed considerably. More advanced UV detectors provide multiple wavelengths at the same time. Besides the simplicity, UV detectors are versatile, sensitive, and reliable with high linearity [7].

\section{Diode array detector}

The diode array detector (DAD) uses a polychromatic light source, which, after passing through a liquid sensor cell and dispersing by a prism or diffraction grating, collects data on a group of photosensitive diodes. Each diode detects a slightly different wavelength than the other which eventually leads to the absorption spectrum for possible identification of the sample [8]. 


\section{Modes of Separation}

Modes of separation mainly depend on the polarity of the stationary and the mobile phases. As a result, there are two general modes of separation, which will be explained below.

\section{Normal phase high performance liquid Chromatography}

In normal phase (NP) chromatography adsorption on the surface of the stationary phase is the principle behind the separation of the analytes. In NP, the stationary phase is relatively polar and the mobile phase is non-polar. As a result, polar analytes tend to interact and stay with the stationary phase longer than the non-polar analytes, hence higher retention time for the polar compounds is expected. Consequently, non-polar analytes tend to elute faster resulting in lower retention time. The mobile phase in NP chromatography usually consists of a non-polar solvent, such as hexane, heptane, chloroform, isopropanol, or ethyl acetate. Alternatively, the stationary phase may have some polar functional groups such as diol, amino, and cyano groups bonded to the surface of the silica $[1,6]$.

\section{Reversed phase high performance liquid chromatography}

Reversed phase (RP) chromatography utilizes a stationary phase that is non-polar and a polar mobile phase. Columns with organic moieties attached to the surface of silica can provide a non-polar stationary phase. The mobile phase consists of polar solvents such as water and soluble organic solvents such as acetonitrile, methanol, and 
tetrahydrofuran (THF). The presence of a polar mobile phase causes the analytes with medium to high polarity to elute first, and inversely those of lower polarity are retained on the stationary phase and elute later. In RP chromatography, partition of solutes into the non-polar phase is the main mechanism for the separation of solutes $[1,6]$.

\section{Aqueous normal phase high performance liquid chromatography}

When a stationary phase possesses both non-polar and polar retention properties, the mode is called aqueous normal phase (ANP) [9]. A study by Pesek, et al. demonstrates that ANP is one of the most versatile separation modes allowing for a wide range of solutes to be analyzed and separated at the same time. More specifically, in ANP, with a binary solvent system, altering the polarity of one of the solvents enables the

column to perform in both the NP and the RP retention modes. A higher concentration of a polar solvent such as water allows the column to operate in the RP mode. When there is a higher concentration of organic solvent, the column has the ability to perform in the ANP mode. Moreover, addition of buffers such as formic acid, ammonium formate, or ammonium acetate in small amounts to the mobile phase enhances the retention of acidic or basic polar analytes $[9,10]$. Another unique feature of ANP is the composition of the stationary phase. In ANP, silica is replaced by silica hydride. The replacement of - $\mathrm{OH}$ functional group by $-\mathrm{H}$, which is done via a silanization process, eliminates many unwanted interactions due to hydrogen bonding in the former or due to electrostatic interaction when the silanols are deprotonated. The ANP mode differs from the somewhat similar hydrophilic interaction chromatography (HILIC). ANP and HILIC are 
distinguished because the former can retain both polar and non-polar analytes in the stationary phase upon altering the composition of mobile phase, whereas the latter is a technique that only deals with the separation of polar compounds [11].

\section{E. Column in HPLC \\ 1. Column efficiency}

There are several factors involved in the performance of a column: resolution, selectivity, the sample capacity factor, and column efficiency. According to equation (1), column efficiency $(\mathrm{N})$ is directly related to the number of theoretical plates:

$$
\mathrm{N}=5.54\left(t_{R} / \mathrm{W}\right)^{2}
$$

$t_{R}=$ the retention time of the solute $\quad \mathrm{W}=$ the width of the peak

The height equivalent to a theoretical plate (HETP) is inversely related to the column efficiency and directly related to the length of the column, L, as described by the Van Deemter equation (2) [1,12].

$$
\mathrm{HETP}=\mathrm{L} / \mathrm{N}
$$

\section{Column packing}

In choosing the right material for the stationary phase, one must consider several important criteria. The durability, inertness, high efficiency, and adequate surface area are some of the physical properties of importance. Some of the packing materials available are silica, zirconia, thoria, alumina, and titania. However, silica (a readily available material with low cost) not only possesses all of the aforementioned properties, 
but also comes in different pore sizes and shapes, an important factor for various industrial applications. The pore size of silica has a direct correlation with the size of the molecules retained on the stationary phase [13]. Therefore, silica is considered to be one of the most widely used packing materials for columns in HPLC. There are, however, some limitations associated with the use of silica. Silica, due to its structure, is somewhat acidic and tends to interact with basic compounds. Additionally, silica is not stable at a $\mathrm{pH}$ lower than 2.0 or higher than 8.0 due to decomposition or dissolution [14].

Silica is a porous material having a chemical composition of $\mathrm{SiO}_{2} \bullet \mathrm{H}_{2} \mathrm{O}$. The structure consists of a network of siloxane bonds ( $\mathrm{Si}-\mathrm{O}-\mathrm{Si})$ and terminal silanol groups (Si-OH). The silanols can exist in geminal or vicinal forms as depicted in Figure 2 [15].

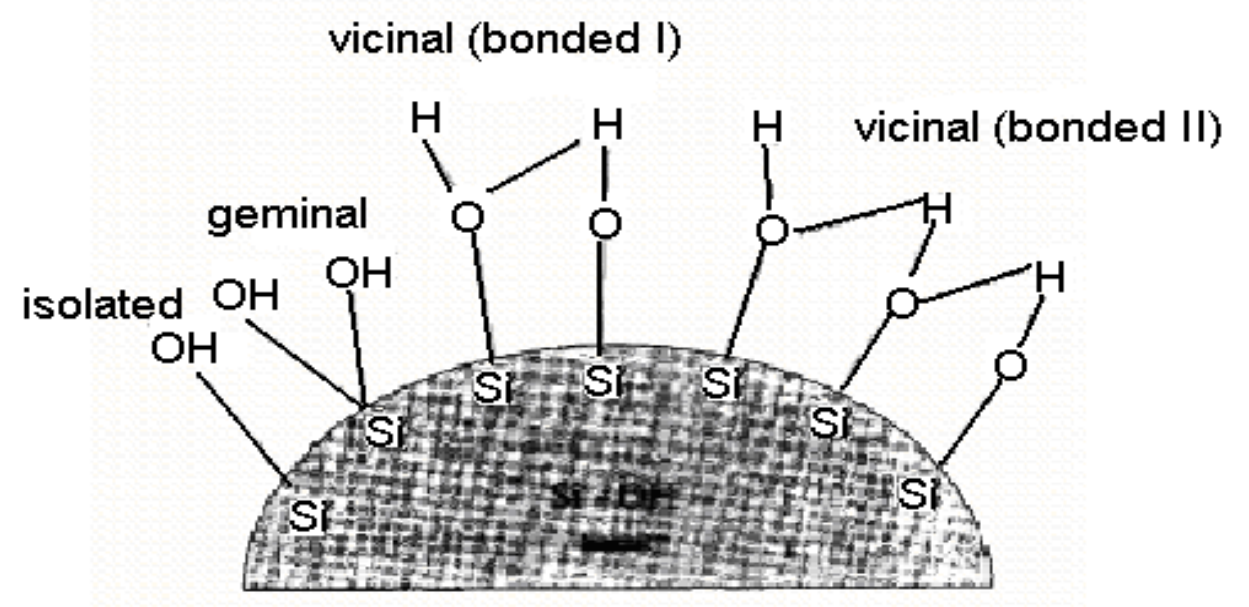

Figure 2. Different types of silanols on the silica surface [15]

It is the presence of the $-\mathrm{OH}$ group that gives silica acidic properties and makes silica less suitable for certain compounds and at certain $\mathrm{pH}$ values. In other words, in RP 
chromatography, when the mobile phase is a binary mixture of organic solvents and $\mathrm{H}_{2} \mathrm{O}$, or in cases when polar compounds are analyzed, there will be some irreversible adsorption of the compounds on the silica surface due to the formation of hydrogen bonds. For instance, the acidic silica can cause unwanted interactions with analytes with basic properties leading to poor efficiency. Moreover, when water is used as part of the mobile phase, it can be adsorbed by the stationary phase (Type B Silica) because of the free $-\mathrm{OH}$ groups on the surface, Figure 3. As the water content on the surface of the stationary phase increases, the retention of analytes often changes [16]. The presence of free silanols also causes peak tailing in the chromatograms.
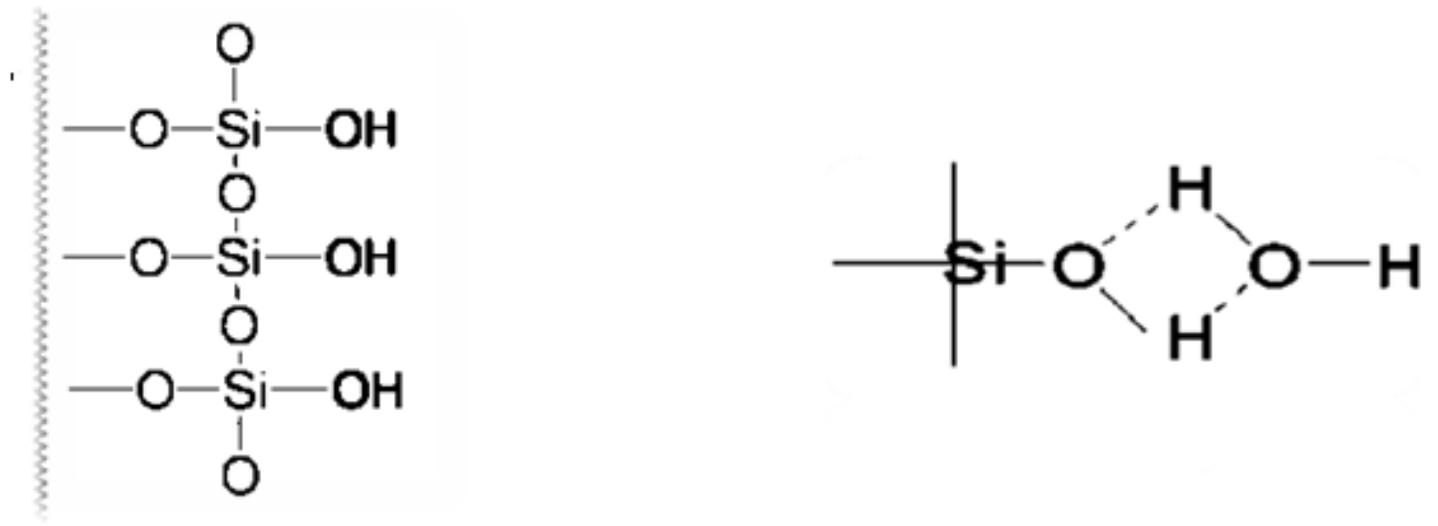

Figure 3. Type B silica and strong water molecule association through hydrogen bonding [16]

\section{Methods to modify silica surface}

In order to overcome the problems caused by the silanol groups on the silica surface, one logical approach is the conversion of the $-\mathrm{OH}$ group to a less active 
functional group such as $-\mathrm{H}$. The conversion prevents the mobile phase water adsorption on the surface, as shown in Figure 4.

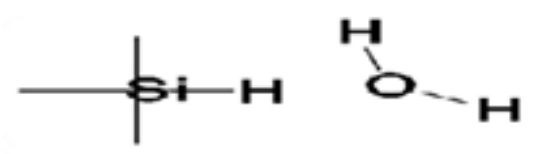

Figure 4. Type C silica and elimination of water molecule [16]

There are several methods proposed to address this problem. Each method comes with its own advantages and disadvantages. The following are the most commonly used approaches.

\section{i. Esterification}

This method, which is similar to an organic esterification method, results in conversion of the $-\mathrm{OH}$ in silanol into an $-\mathrm{OR}$ using an alcohol as the R-group donor, as shown in reaction (1). This material undergoes $\mathrm{Si}-\mathrm{O}-\mathrm{R}$ bond breakage, under acidic hydrolytic conditions, and is not suitable for most chromatographic applications [17].

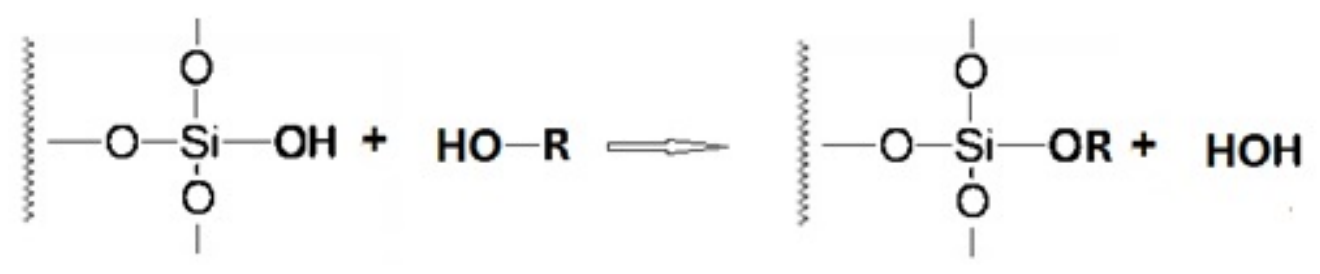




\section{ii. Organosilanization}

Another approach to remove the acidic - $\mathrm{OH}$ group of silanol is organosilanization. As shown in reaction (2), a dimethylalkyl silane (X-SiR' $\left.{ }_{2} \mathrm{R}\right)$ replaces the $-\mathrm{OH}$ in silanol [18]. The formation of an $\mathrm{Si}-\mathrm{R}$ bond is very desirable in terms of introducing various R groups; hence, multiple stationary phases can be produced.

However, the HX byproduct can cause the hydrolysis of the Si-R bond and unwanted results. Moreover, if the conversion of all $\mathrm{Si}-\mathrm{O}$ bonds to $\mathrm{Si}-\mathrm{R}$ is not completely successful the presence of silanols on the surface could cause irreversible adsorption between the analytes and the stationary phase [18].

In the second type of organosilanization, as shown in reaction (3), a trifunctional organosilane reacts with the surface silanols followed by a crosslinking, which results in the formation of a bonded stationary phase [19]. The disadvantage of this approach could be the significant reduction in the interactions between basic solutes and silanols [19].

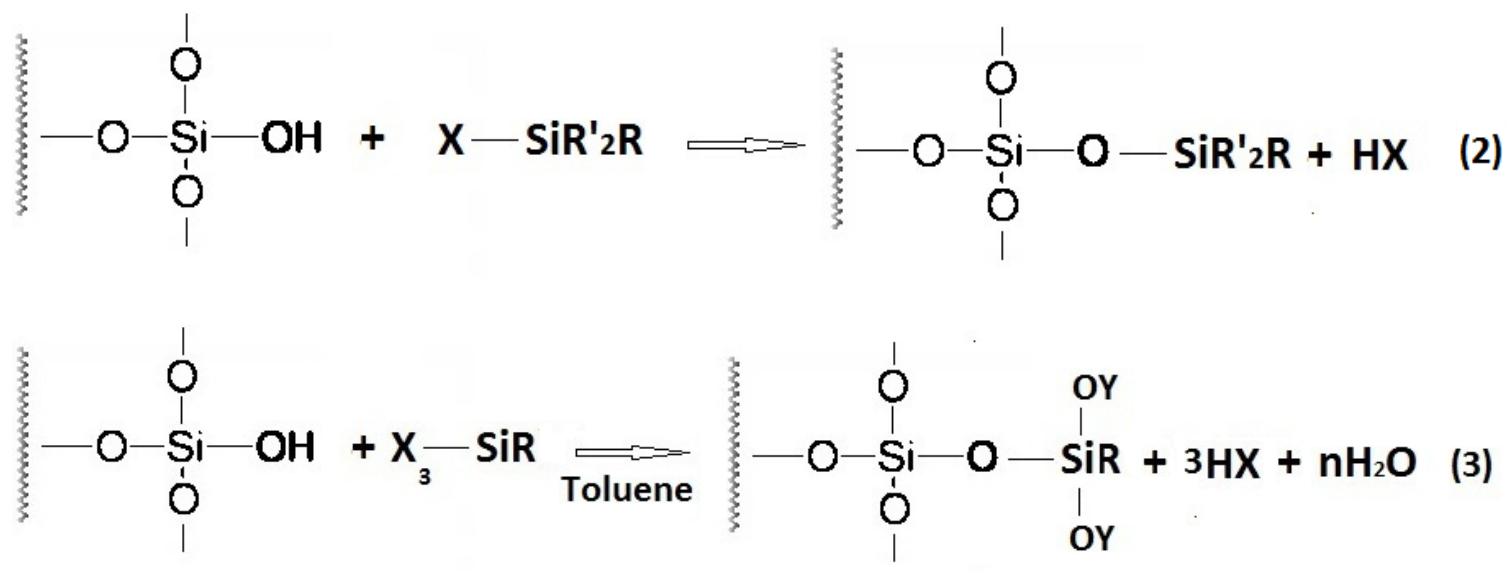

where $\mathrm{n}=1-3$ and $\mathrm{Y}=\mathrm{H}$ or $\mathrm{Si}(\mathrm{Osi})_{2} \mathrm{R}$ based on the extent of crosslinking [19]. 


\section{iii. Grignard Reaction}

A Grignard reaction involves addition of thionyl chloride in the presence of toluene to form a silica-halide intermediate, as described in reaction (4). Addition of an organolithium reagent or a Grignard reagent to this intermediate results in the formation

of a Si-R bond and removal of the silanol groups, as shown in reaction (5). Although the reaction replaces almost all of the silanol groups, this method suffers from acidic hydrolysis of the intermediate [18].

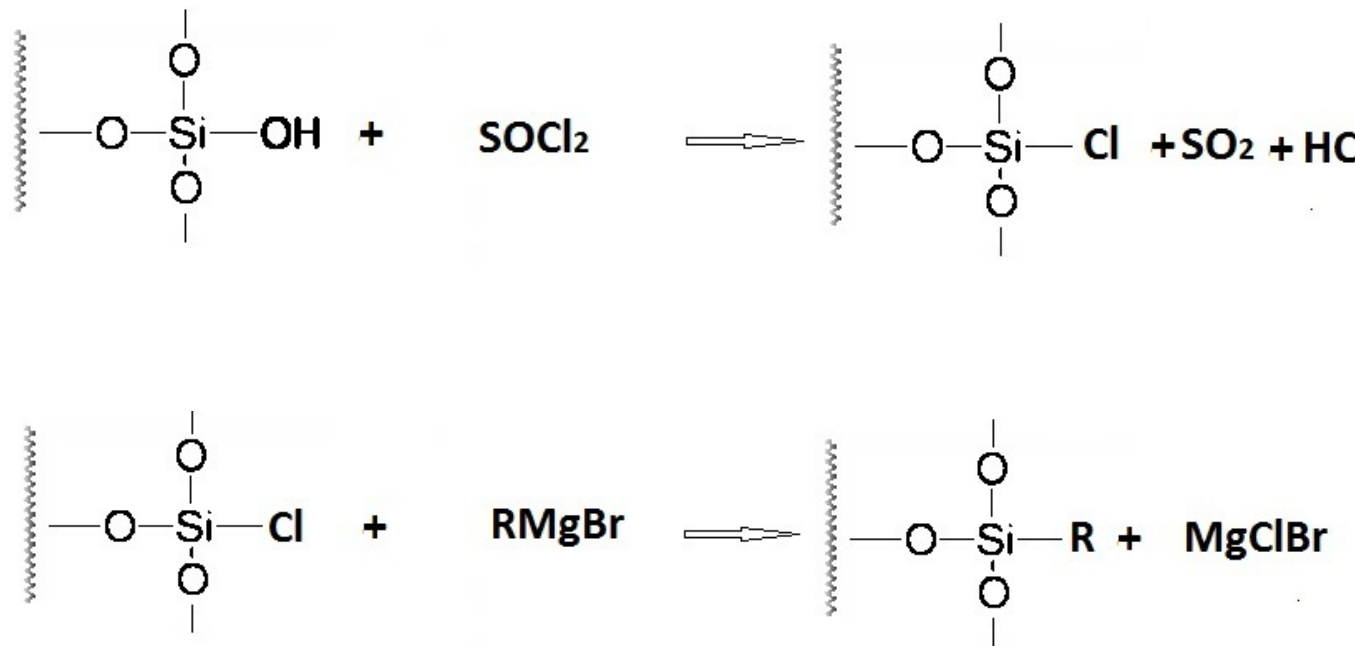

\section{iv. Silanization/ Hydrosilation}

Introduced first by Pesek, et al., this novel technique has addressed many of the disadvantages of silica. The technique has also opened the door to the production of a variety of stationary phases for different purposes. There are two major steps involved in this method. The first step is the silanization reaction. Following a successful silanization, hydrosilation, the second step of the procedure, will complete the process. 
Silanization involves the removal of the $-\mathrm{OH}$ group from silica and replacing it with hydrogen, hence the formation of silica hydride. This results in the formation of what is commercially known as Type-C silica instead of Type-B silica. A schematic comparison of the two types is given in Figure 5. As shown in reaction (6), the $\mathrm{Si}-\mathrm{OH}$ bond converts to $\mathrm{Si}-\mathrm{H}$ in the presence of triethoxy-silane (TES) and an aqueous $\mathrm{HCl}$ catalyst. Studies have shown that the hydride product obtained from silanization is stable and does not undergo a hydrolysis reaction [20]. Moreover, the modification is efficient in replacing more than 95\% of the silanol groups [14].

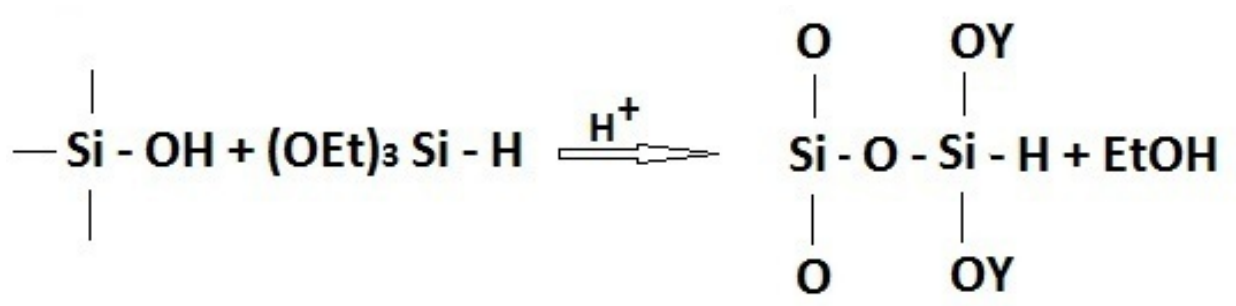

\section{$\mathbf{Y}=\mathbf{H}$ or $\mathbf{S i}$}

Recent studies have shown that the minimal steric hindrance of the small $\mathrm{Si}-\mathrm{H}$ group, which could result in a large Si-H population on the surface of silica, could also cause formation of multilayered $\mathrm{SiH}$ groups. The drawbacks were mainly observed when TES was involved as one of the reactants and water as a catalyst [21]. 
TYPE-B SILICA

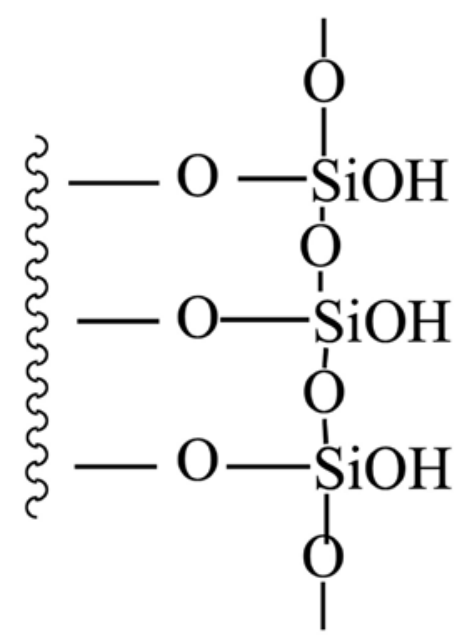

TYPE-C SILICA

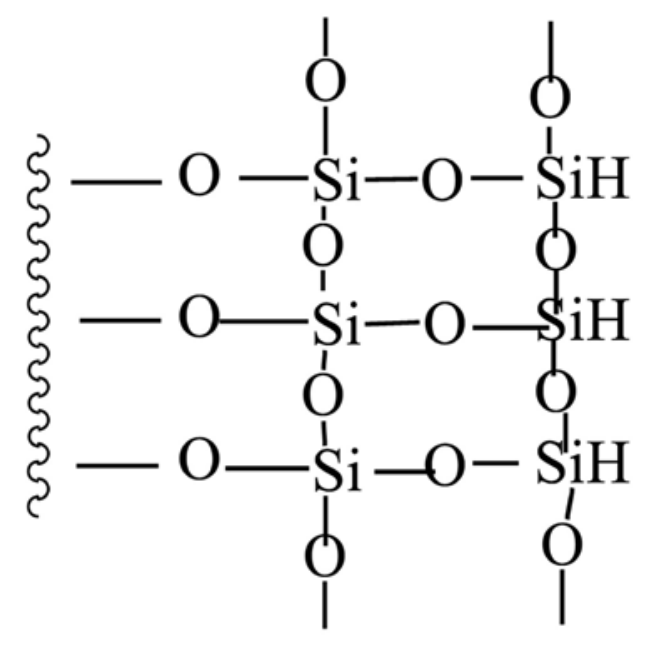

Figure 5. Schematic comparison of Type-B vs. Type-C silica [14]

To resolve this issue, Sandoval has suggested two different methods. In the first approach, silica is reacted with $\mathrm{HSiCl}_{3}$, trichlorosilane (TCS), dissolved in an organic solvent such as toluene [22]. In their second approach, silica is reacted with TES, $\mathrm{HSi}(\mathrm{OEt})_{3}$ in a small amount of aqueous $\mathrm{HCl}$ dissolved in THF [21].

Hydrosilation is the introduction of an organic group to the surface of silica hydride from silanization. Depending on the structures and properties of the added groups to the surface, hydrosilation produces stationary phases with various properties suitable for hydrophilic, hydrophobic, or ion exchange chromatography. Through this process, an organic moiety attaches to the surface of silica hydride forming a stable ( $\mathrm{Si}-$ C) bond. In hydrosilation the silica hydride and a terminal olefin, the most used form of organic compounds, react using hexachloroplatinic acid in 2-propanol, also known as Speier's catalyst, as shown in reaction (7). 


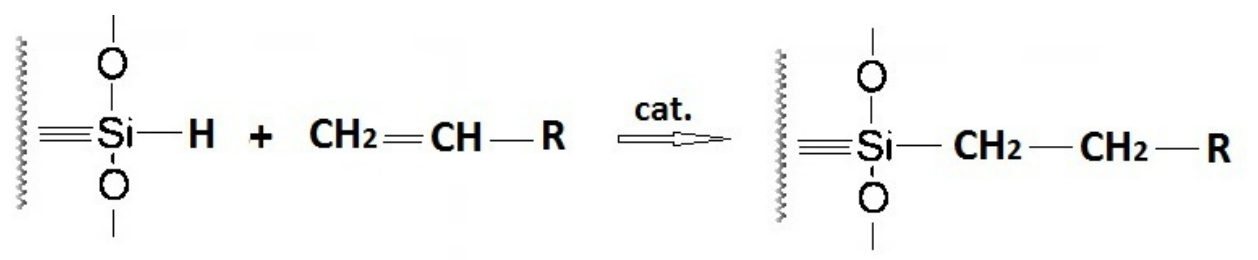

One of the drawbacks of this reaction is perhaps the use of hexachloroplatinic acid in 2-propanol. The Speier's catalyst available in this reaction can produce intermediate species with the organic compound. This reaction could in turn result in lower product yield and efficiency of the reaction. Additionally, platinum metal can deposit on the surface of the silica hydride and reduce the efficiency [23]. There have been studies that have suggested alternative catalysts such as azobisisobutyronitrile (AIBN) for the hydrosilation reactions [23].

Although terminal olefins are the most commonly used organic moieties in hydrosilation, recent studies have led to the development of reactions using alkynes [24], cyano compounds [25], and non-terminal olefins [26]. For instance, structures I and II in reaction (8) can be considered as two proposed bonding structures in the synthesis of a stationary phase from hydrosilation of silica hydride with an alkyne.
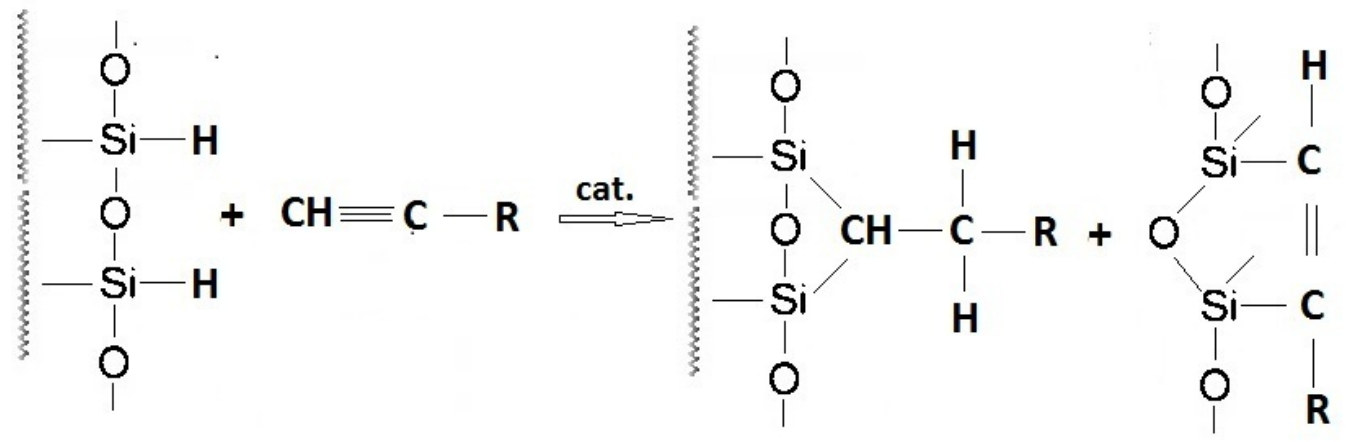

Structure I

Structure II 


\section{F. Research Goals}

In this study, silica hydride was the base material for the stationary phases used in HPLC. However, attachment of various moieties to the surface of the silica hydride results in a stationary phase better adapted to either reverse phase (RP) or aqueous normal phase (ANP) characteristics. The objective of many studies has been to create columns that are responsive to both conditions, RP and ANP, at the same time. The focus of this study was also to introduce novel organic moieties to the surface of the silica hydride and, therefore, to develop columns that can perform under both reverse phase (RP) and aqueous normal phase (ANP) conditions without the need to exchange columns when switching from one condition to the other. To verify the accomplishment of the goal, each column was then subjected to a variety of hydrophilic and hydryophobic analytes as well as polar and non-polar mobile phases for retention behavior studies.

The first part of the study was to synthesize the stationary phases. To obtain HPLC columns, which are amenable to many applications, silica was initially converted to silica hydride through the silanization method, as explained above. FTIR was used to characterize the material and confirm that $\mathrm{Si}-\mathrm{H}$ groups were in abundance on the surface. The next phase was hydrosilation through which five carefully chosen organic compounds were introduced onto the silica hydride surface. In the hydrosilation reactions, $m$-dimethylbenzene, ethynylanaline, 1,8-nonadiyene, 6-chloro-1-hexyne, and 5-cyano-1-pentyne were bonded to the surface of silica hydride through the aromatic ring in m-dimethylbenzene and alkyne groups in other compounds. Upon completion of each reaction, the newly synthesized stationary phases were subjected to spectroscopic 
characterization and determination of percent surface coverage. To confirm the success of each hydrosilation, the samples were spectroscopically evaluated using FTIR, ${ }^{13} \mathrm{C}$ NMR, and ${ }^{29}$ Si-NMR. Carbon analyses for each sample measured the percent surface coverage. Following the evaluations, it was discovered that $m$-dimethylbenzene did not result in a reasonable surface coverage and therefore was removed from the rest of the study. Each of the other four compounds was used as a stationary phase and packed in a stainless steel HPLC column.

The next phase in this research was to evaluate each column for its behavior towards hydrophilic and hydrophobic analytes under a wide range of polar and non-polar mobile phases. This study was done by selecting analytes from different categories to observe the retention behavior of each under RP and ANP conditions.

The goal for this study was to design a stationary phase(s) that contains both RP and ANP modes simultaneously. When using a mixture of polar and not-polar solvents as the mobile phase, an ideal retention time graph for such a column has a U-shape curve, as illustrated in Figure 6. This behavior could be interpreted as the polarity of the mobile phase decreases the stationary phase shifts toward the ANP mode. This would result in a longer retention time for hydrophilic compounds and vice versa.

With that objective in mind, four bonded stationary phases were synthesized, characterized, and evaluated for their RP/ ANP mode using a wide variety of polar and non-polar analytes. 


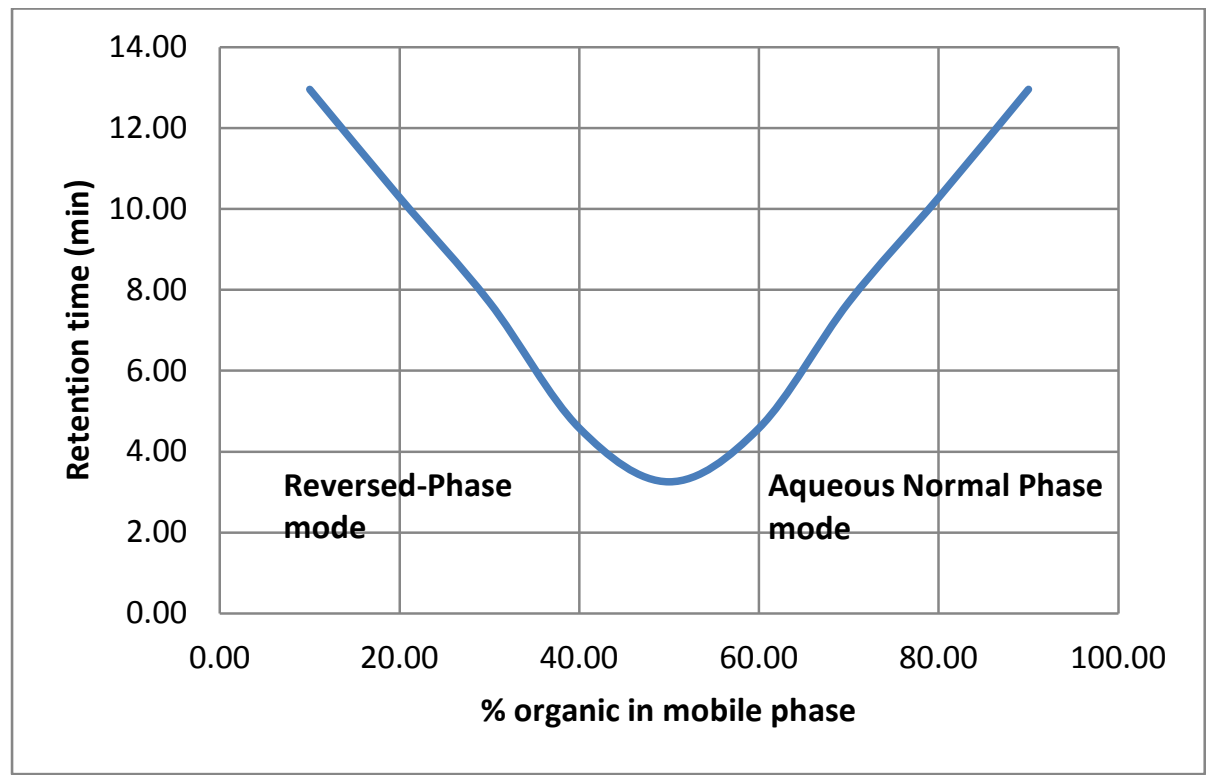

Figure 6. Ideal RP and ANP regions retention map on Si-H stationary phase 


\section{EXPERIMENTAL}

\section{A. Materials}

\section{Silanization: Preparation of silanol}

The materials used for this part are listed in the following tables. The main compound in the production of stationary phases was silica. AstroSil ${ }^{\circledR}$ Silica was obtained from Stellar Phases (Langhorne, PA).

Table 1. Specfications of AstroSil ${ }^{\circledR}$ silica.

\begin{tabular}{|l|l|}
\hline Lot Number & $7-122 \mathrm{FA}$ \\
\hline Pore Size (nitrogen, average pore) & $98 \AA$ \\
\hline Pore Volume & $0.9 \mathrm{~mL} / \mathrm{g}$ \\
\hline Surface Area (nitrogen, BET multipoint) & $345 \mathrm{sq} . \mathrm{m} / \mathrm{g}$ \\
\hline Apparent Density (tap density) & $0.45 \mathrm{~g} / \mathrm{mL}^{*}$ \\
\hline Particle Size (microscopic analysis) & $5.2 \mu$ \\
\hline Particle Distribution dp 90/dp 10 & 1.6 \\
\hline \multicolumn{1}{|c|}{ Metal Content (ICP scan, HF digest) } \\
\hline Na & $<4 \mathrm{ppm}$ \\
\hline $\mathrm{Mg}$ & $<10 \mathrm{ppm}$ \\
\hline Ca & $<10 \mathrm{ppm}$ \\
\hline Al & $<2.8 \mathrm{ppm}$ \\
\hline Fe & $<2.7 \mathrm{ppm}$ \\
\hline
\end{tabular}

*Tap density is based on $15 \mu$ m particles. Values for smaller particles will be higher. 
Table 2. Compounds used for the silanization.

\begin{tabular}{|c|c|c|}
\hline Compound & CAS number & Manufacturer \\
\hline Silica & & Stellar Phases \\
\hline Triethoxysilane (TES) & $78-07-09$ & Sigma-Aldrich \\
\hline Dioxane & $123-91-1$ & Fisher Chemicals \\
\hline Hydrochloric acid, 2.3M & $7647-01-0$ & Sigma-Aldrich \\
\hline Diethylether & $60-29-7$ & Aldrich \\
\hline Tetrahydrofuran (THF) & $142-68-7$ & \\
\hline
\end{tabular}

\section{Hydrosilation: Preparation of stationary phase}

In this step, the silanols were converted into silica hydrides. The conversion was followed by introduction of five organic moieties on the surface of silica hydride for a novel stationary phase. Each organic moiety was bonded individually. 
Table 3. Compounds used for hydrosilation.

\begin{tabular}{|c|c|c|}
\hline Compound & CAS number & Manufacturer \\
\hline Hexachloroplatinic acid & $16941-12-1$ & Sigma-Aldrich \\
\hline Toluene & $108-88-3$ & Fisher Chemicals \\
\hline Diethylether & $60-29-7$ & Sigma-Aldrich \\
\hline AstroSil ${ }^{\circledR}$ hydride & & hydrosilation AstroSil $^{\circledR}$ silica \\
\hline 1,8-Nonadiyne & & GFS Chemicals, Inc. \\
\hline$m$ - Diethynylbenzene & & GFS Chemicals, Inc. \\
\hline 5-Cyano-1-pentyne & & GFS Chemicals, Inc. \\
\hline 6-Chloro-1-hexyne & & GFS Chemicals, Inc. \\
\hline 4-Ethynylaniline & & GFS Chemicals, Inc. \\
\hline
\end{tabular}

\section{HPLC: Mobile phase preparation}

The chromatographic evaluation of each stationary phase utilized different concentrations of an organic solvent, acetonitrile, and a polar solvent, MilliQ $\mathrm{H}_{2} \mathrm{O}$. In addition to the mentioned solvents, small volumes of an additive, formic acid, was used in some cases. 
Table 4. Compounds used in the mobile phase.

\begin{tabular}{|c|c|c|}
\hline Compound & CAS number & Manufacturer \\
\hline Acetonitrile HPLC grade & $75-05-8$ & Fisher Chemicals \\
\hline MilliQ DI Water & & Millipore \\
\hline Formic Acid & $64-18-6$ & Spectrum Mfg. Corp. \\
\hline
\end{tabular}

\section{HPLC analysis: Compounds analyzed with the stationary phases}

The following tables, 5, 6, 7, 8, and 9 represent the analytes used in this study.

Table 5. Amino acids (hydrophilic/ polar).

\begin{tabular}{|c|c|c|}
\hline Compound & CAS number & Manufacturer \\
\hline L-(+)- $\alpha$-Phenylglycine 99\% & $2935-35-5$ & Sigma-Aldrich \\
\hline Phenylalanine & $20700-34-3$ & Sigma-Aldrich \\
\hline
\end{tabular}

Table 6. Nucleobases (hydrophilic/ polar).

\begin{tabular}{|c|c|c|}
\hline Compound & CAS number & Manufacturer \\
\hline Adenine & $73-24-5$ & Sigma-Aldrich \\
\hline Guanine & & NBC National Biochemical \\
& & Corp. \\
\hline
\end{tabular}


Table 7. Neurotransmitters (hydrophilic/ polar).

\begin{tabular}{|c|c|c|}
\hline Compound & CAS number & Manufacturer \\
\hline Epinephrine & $51-43-4$ & Sigma \\
\hline Norepinephrine & $51-41-2$ & Sigma \\
\hline Dopamine & $62-31-7$ & Sigma \\
\hline
\end{tabular}

Table 8. Steroids (hydrophobic/ non-polar).

\begin{tabular}{|c|c|c|}
\hline Compound & CAS number & Manufacturer \\
\hline Adrenosterone & $382-45-6$ & Sigma-Aldrich \\
\hline$\Delta^{4}$-Androsten-3,17-dione & $63-05-8$ & Sigma-Aldrich \\
\hline Estrone & $53-16-7$ & Sigma-Aldrich \\
\hline Estradiol & $50-28-2$ & Sigma-Aldrich \\
\hline Corticosterone & $50-22-6$ & \\
\hline
\end{tabular}

Table 9. Polycyclic aromatic hydrocabons (PAH) (hydrophobic/ non-polar).

\begin{tabular}{|c|c|c|}
\hline Compound & CAS number & Manufacturer \\
\hline Naphthalene & $91-20-3$ & Sigma-Aldrich \\
\hline Phenanthrene & $85-01-8$ & Sigma-Aldrich \\
\hline Pyrene & $129-00-0$ & Aldrich \\
\hline Fluorene & $86-83-7$ & Aldrich \\
\hline
\end{tabular}




\section{B. Structures}

1. Organic compounds used in the synthesis of the bonded stationary phase

There were five organic compounds designed to be introduced to the surface of stationary phases. The structure of these compounds are illustrated below.

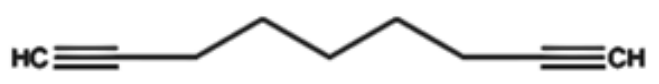

1,8-Nonadiyne

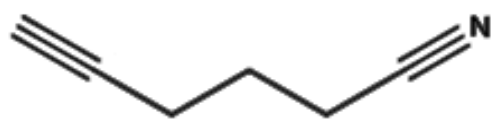

5-Cyano-1-pentyne

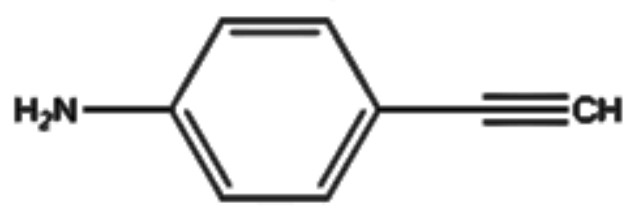

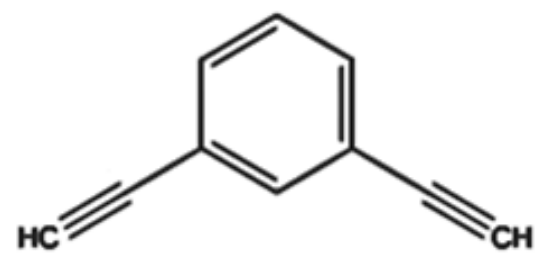

m-diEthynylbenzene

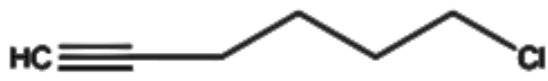

6-Chloro-1-hexyne

\section{4-Ethynylanaline}




\section{Analytes used for the HPLC evaluations}

\section{i. Amino acids}

Phenylalanine and Phenylglycine were the two amino acids used as polar anayltes in this study. The structures of the two amino acids are shown below.

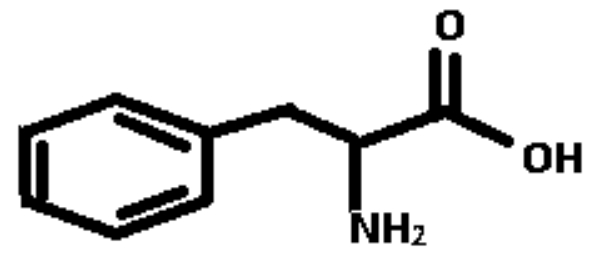

Phenylalanine

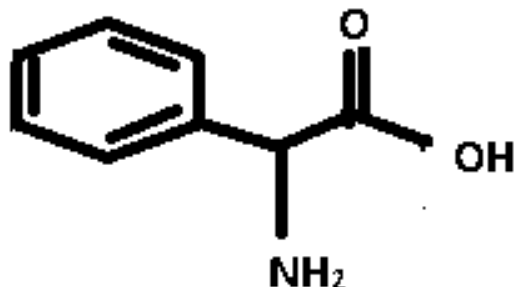

Phenylglycine

\section{ii. Nucleobases}

For the nucleobases, Adenine and Guanine with the structures shown below were used.

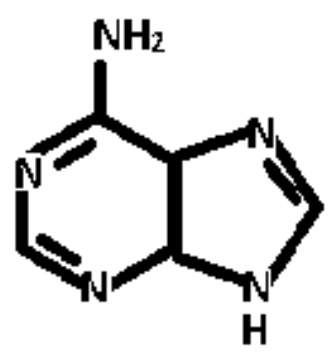

Adenine

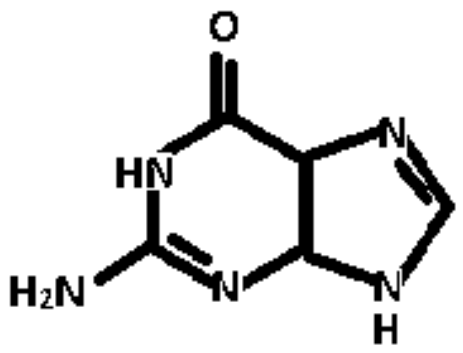

Guanine 


\section{iii. Neurotransmitters}

The other set of polar compunds used as analytes were the neurotramsitters, Epinephrine, Norepinephrine, and Dopamin. The structers to these compounds are shown below.

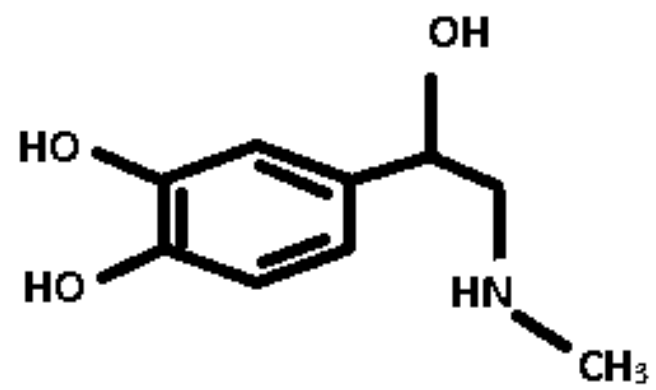

Epinephrine

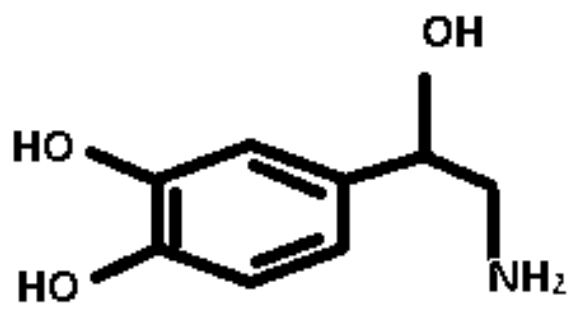

Norepinephrine

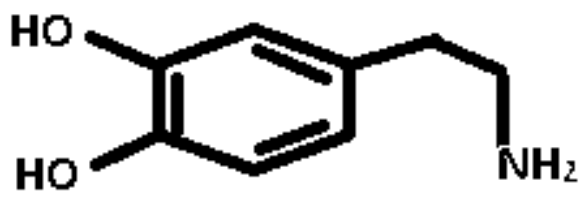

Dopamine 


\section{iv. Steroids}

Adrenosterone, Androsten-3,17-dione, Esterone, Estradiol, and Corticosterone were five non-polar analytes from the steroid family. The structre of each analyte is shown below.

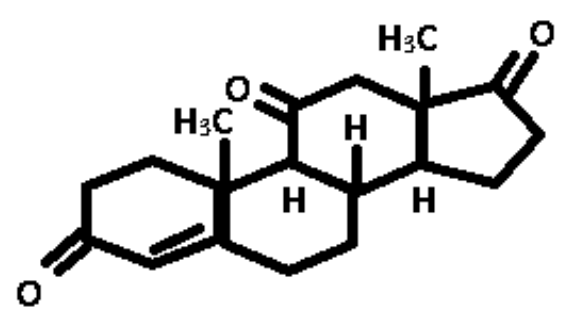

Adrenosterone

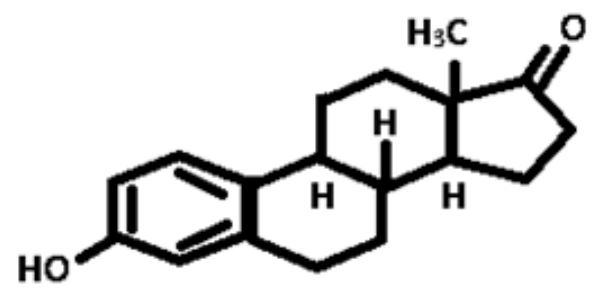

Esterone

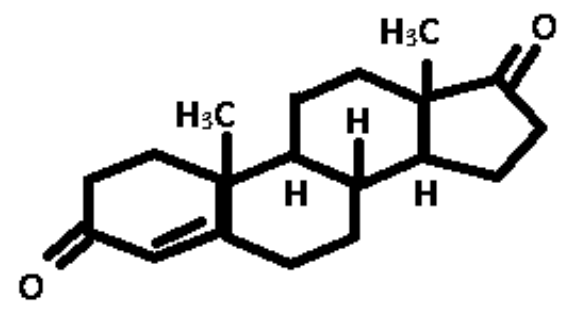

Androsten-3,17-dione

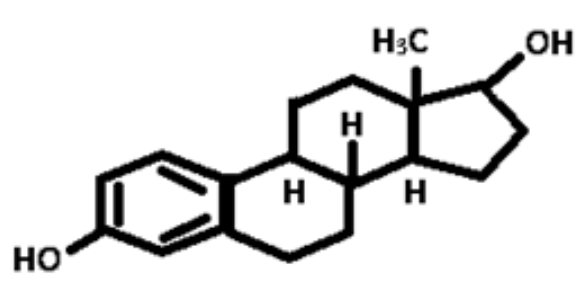

Estradiol 


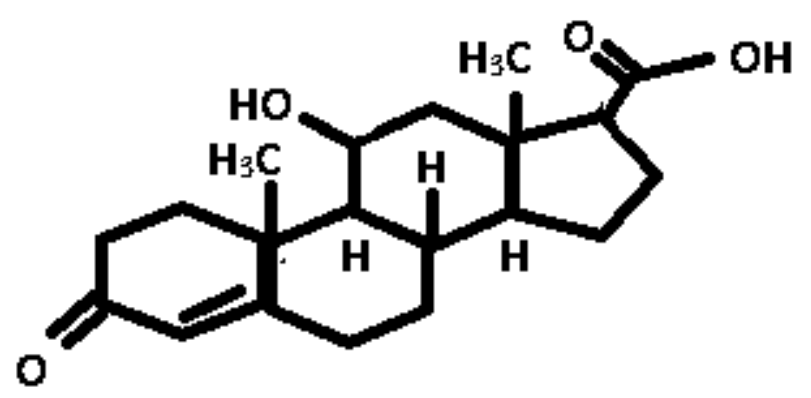

\section{Corticosterone}

\section{v. Polycyclic Aromatic Hydrocarbons}

The poly aromatic hydrocarbons, Naphthalene, Phenanthrene, Pyrene, and Flourene were used as another group of non-polar analytes. The structure of each analyte is shown below.

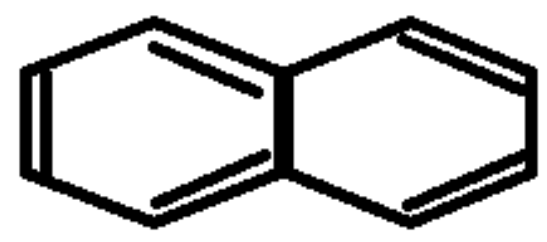

Naphthalene

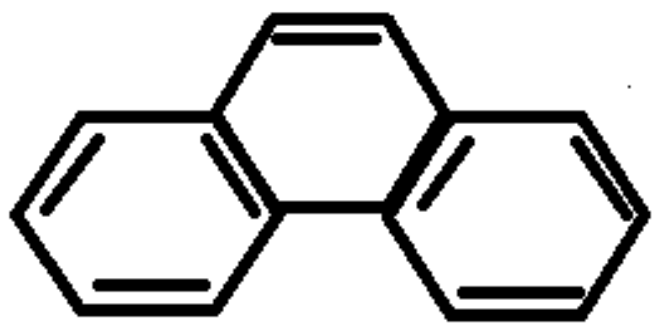

Phenanthrene 


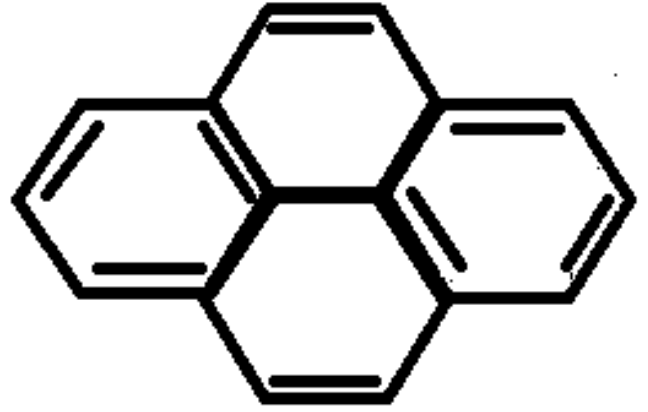

Pyrene

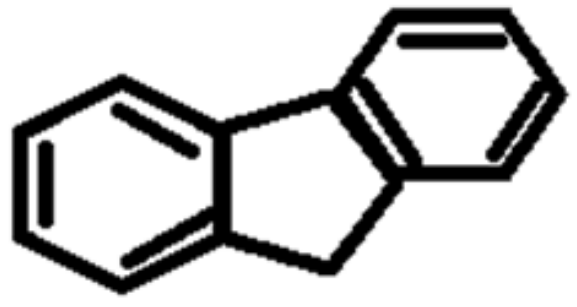

Fluorene 


\section{Methods}

\section{Silanization: Synthesis of silica hydride}

Silanization must be performed under strictly controlled conditions. Therefore, it was necessary to oven-dry all equipment for $24 \mathrm{hr}$ after careful washing and cleaning. In order to synthesize silica hydride, 25 g of Astrosil Silica ${ }^{\mathrm{TM}}$, stored and dried overnight in a vacuum oven at $120{ }^{\circ} \mathrm{C}$, was weighed. The oven’s vacuum pump has a controlled pressure of apporoximately -29 inHg or higher. The dried silica was transferred to a 2000 $\mathrm{mL}$ three-necked round bottom flask which contained $900 \mathrm{~mL}$ dioxane and $32.4 \mathrm{~mL}$ of 2.30 $\mathrm{M} \mathrm{HCl}$ as a catalyst. To ensure complete transfer of silica, $100 \mathrm{~mL}$ of dioxane was used to wash the remaining silica into the flask. The Astrosil Silica ${ }^{\mathrm{TM}}$, dioxane, and $\mathrm{HCl}$ mixture was heated up to $70{ }^{\circ} \mathrm{C}$. To facilitate this procedure, the round bottom flask was attached to a condenser and placed on top of a heating mantel. The entire setup was connected to a stirring apparatus. The reaction temperature was controlled by a thermometer, placed inside the three-necked flask through a rubber stopper, and stirring was done by a magnet placed inside the flask.

Once the mixture reached and remained constant at $70{ }^{\circ} \mathrm{C}, 35.0 \mathrm{~mL}$ of pure triethoxysilane, TES, mixed with $150.15 \mathrm{~mL}$ dioxane was added to the solution, in a drop-wise manner with constant stirring in the presence of argon gas, using an addition funnel. Upon completion, the temperature of the mixture was raised to $90{ }^{\circ} \mathrm{C}$ in about 30 min and maintained at that temperature for $90 \mathrm{~min}$. The silica hydride product was separated from the solution using a vacuum filtration method with an oven-dried $90 \mathrm{M}$ sintered funnel. The solid silica hyride was washed three times, using $90 \mathrm{~mL}$ dioxane, 90 
mL DI water, and $27.63 \mathrm{~mL}$ tetrahydrofuran per washing. To ensure complete removal of TES, $90 \mathrm{~mL}$ of diethylether was used for final washing. The silica hydride was then dried and kept in a vaccum oven at a temperature of $110{ }^{\circ} \mathrm{C}$ for 24 hours [20]. The total weight of the final product was 21.00 grams, and the productwas characterized using the FTIR method.

\section{Hydrosilation: Synthesis of stationary bonded phase}

The hydrosilation reaction consisted of two major steps. The initial step was the activation of the selected organic moiety designed to be bonded to the stationary phase. For the second step, the silica hydride and the activated organic moiety were combined to form a new bonded stationary phase.

The approach for the hydrosilation portion of this study was to introduce five selected organic compounds to the surface of silica hyrdride in order to synthesize five unique stationary bonded phases. The reaction procedures for each stationary phase are described in the following sections.

\section{i. Preparation of 1,8-nonadiyne bonded phase}

The hydrosilation reaction, similar to silinization, required the use of oven-dried (temperature of $100{ }^{\circ} \mathrm{C}$ ) equipment. The organic moiety 1,8-nonadiyne$\mathrm{HCC}\left(\mathrm{CH}_{2}\right)_{5} \mathrm{CCH}$ - with the formula weight of $120.20 \mathrm{~g} / \mathrm{mol}$ and structure shown in section (B) was used for this particular reaction. After setting up a three-necked round bottom flask and a condenser placed on top of a heating mantel/ transformer, $195 \mathrm{~mL}$ 
toluene, $0.675 \mathrm{~mL}$ of $10 \mathrm{mM} \mathrm{H}_{2} \mathrm{PtCl}_{6}$ in 2-propanol (Speier's Catalyst), and $4.08 \mathrm{~g}$ of 98\% pure 1,8-nonadiyne were transferred to the flask. The temperature was set to $70{ }^{\circ} \mathrm{C}$ and the reaction mixture was stirred. To ensure complete actiavtion of the new complex, the reaction was allowed to remain at this temperature for one hr. Completion of the first step resulted in formation of a clear solution.

Addtion of $5 \mathrm{~g}$ silica hydride (synthesized from the silanization reaction and stored in a vacuum oven at $120^{\circ} \mathrm{C}$ ) to the solution was the beginning of the second step of the hydrosilation reaction. The silica hydride addition to the flask was done gradually and in small portions with steady stirring. The residual silica was washed with $5 \mathrm{~mL}$ of toluene into the solution. The temperature was then raised to $100{ }^{\circ} \mathrm{C}$ where it was held for $96 \mathrm{hr}$. Upon completion of the reaction, the solid product was separated from the rest of the solution using a $90 \mathrm{M}$ oven-dried sintered glass funnel. The final step was to wash the solid product with $25 \mathrm{~mL}$ of toluene at $90{ }^{\circ} \mathrm{C}$ three times, bringing the total volume to $75 \mathrm{~mL}$, followed by 2 washes of $25 \mathrm{~mL}$ dietheyl ether. Upon completion of the filtration, the remaining washing solvent dietheyl ether was allowed to evaporate from the product by keeping the product at room temperature over night. Complete removal of excess solvents from the product was achieved by vacuum drying at $110{ }^{\circ} \mathrm{C}$ for $24 \mathrm{hr}$ [27].

The characterization of 5.2735 g 1,8-nonadiyne bonded phase was done by three methods: FTIR, carbon analysis, and solid state NMR. The results will be discussed in the following sections. Theoretically, the hydrosilation of silica hydride using 1,8nonadiyne could yield the following product as shown in reaction (9). 


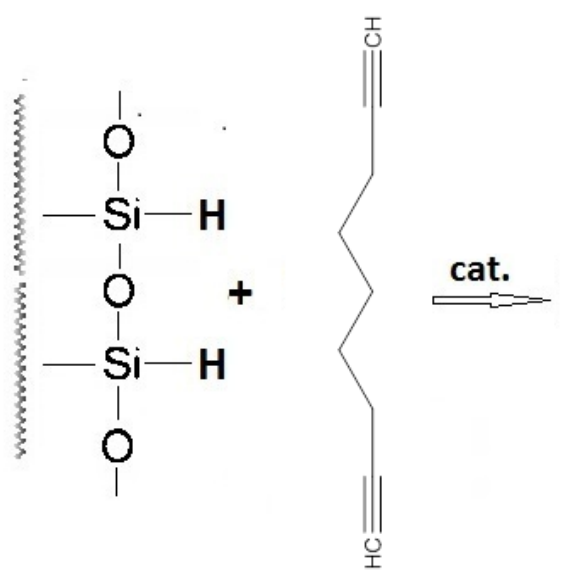

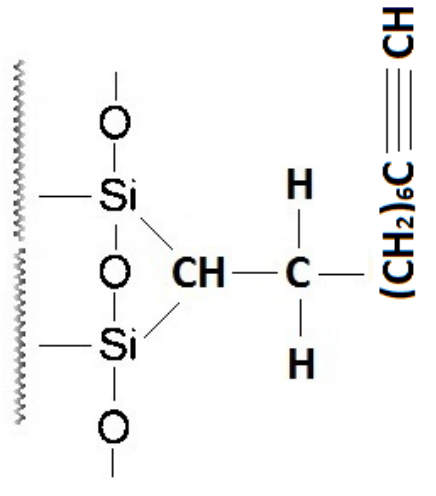

One possible structure

\section{ii. Preparation of $\boldsymbol{m}$-diethynylbenzene bonded phase}

The next organic group selected for a bonded stationary phase was m-diethynylbenzene- $\mathrm{C}_{6} \mathrm{H}_{4}(\mathrm{CCH})_{2}$. This compound with a molecular weight of $126.15 \mathrm{~g} / \mathrm{mol}$ and the structure shown in Section B, was bonded using a hydrosilation procedure similar to that of 1,8-nonadiyene that was described in the previous section. For the experimental procedure, 5.0365g of $m$-diethynylbenzene was added into a threenecked round bottom flask containing $195 \mathrm{~mL}$ toluene and $0.675 \mathrm{~mL} \mathrm{H}_{2} \mathrm{PtCl}_{6}$. The remainder of the $m$-diethynylbenzene was transferred into the flask using $5.0 \mathrm{~mL}$ washing toluene. Upon formation of the activated complex, $5.0 \mathrm{~g}$ silica hydride was added to the mixture. The solution was allowed to react for 96 hours at $100{ }^{\circ} \mathrm{C}$ followed by washing and filtration [27]. The solid product weighed $5.3424 \mathrm{~g}$. To characterize and confirm the presence of the bonded organic compound on the surface of the stationary phase, FTIR, solid state NMR, and carbon analysis were used. 


\section{iii. Preparation of 5-cyano-1-pentyne bonded phase}

5-Cyano-1-pentyne, $\mathrm{HCC}\left(\mathrm{CH}_{2}\right)_{3} \mathrm{CN}$ with the formula weight of $93.13 \mathrm{~g} / \mathrm{mol}$ and structure shown in Section B, was bonded via hydrosilation. This compound (3.7200 g), as well as $0.675 \mathrm{~mL}$ Speier’s catalyst, $200 \mathrm{~mL}$ toluene, and $5.0 \mathrm{~g}$ silica hydride were allowed to react for 96 hours at $100{ }^{\circ} \mathrm{C}$. Following filtration, the solid product was washed three times with $75 \mathrm{~mL}$ (total volume) of toluene at $90{ }^{\circ} \mathrm{C}$ and two times with 50 $\mathrm{mL}$ (total volume) diethyl ether [27]. The final result was a yellow solid with a total weight of $4.9064 \mathrm{~g}$. The newly synthesized stationary phase was characterized for the presence of 5-cyano-1-pentyne using FTIR, carbon analysis, and solid state NMR spectroscopy. Results will be discussed in the following sections.

\section{iv. Preparation of 6-chloro-1-hexyne bonded phase}

The organic moiety 6-chloro-1-hexyne, $\mathrm{Cl}\left(\mathrm{CH}_{2}\right)_{4} \mathrm{CCH}$, with a formula weight of $116.59 \mathrm{~g} / \mathrm{mol}$ and structure shown in Section B, was chosen to be bonded to the surface of silica hydride. Using 4.6500 g 6-chloro-1-hexyne, 0.675 of mL Speier’s catalyst, 200 $\mathrm{mL}$ of toluene, and $5.0 \mathrm{~g}$ of silica hydride, the hydrosilation reaction proceeded under similar conditions as described in the above sections [27]. The result was the production of $5.3953 \mathrm{~g}$ of a new bonded phase material. FTIR, carbon analysis, and solid state NMR spectroscpy were used to characterize the synthesized 6-chloro-1-hexyne bonded phase material. Results will be discussed in the following sections. 


\section{v. Preparation of 4-ethynylanaline bonded phase}

The hydrosilation reaction for 4-ethynylanaline, $\mathrm{C}_{8} \mathrm{H}_{7} \mathrm{~N}$, an organic compound with a formula weight of $117.15 \mathrm{~g} / \mathrm{mol}$ and structure shown in Section B, was bonded to the silica hydride according to the standard procedure as described in the earlier sections [27]. For this reaction, $4.68 \mathrm{~g}$ of $98 \%$ pure ethynylanaline was used and the result was the production of $0.1278 \mathrm{~g}$ of a yellow-colored solid. Similar characterization techniques, FTIR, carbon analysis, and solid state NMR spectroscopy were applied for verification of the newly synthesized bonded phase. Results will be discussed in the following sections.

\section{Column packing}

Upon characterization and confirmation of the bonded stationary phases, the materials were subsequently utilized for column packing. The results obtained from FTIR spectorscopy and carbon analysis did not provide compelling evidence that m-diethynylbenzene had an acceptable surface coverage; hence the material did not appear to be a promising packing material. Column packing involves loading the synthesized bonded phase under high pressure into a stainless steel column for further chromatographic experiments.

The column packing process for 1.8029 g 1,8-nonadiyne bonded phase was performed at San José State University by Dr. Pesek. This process involved a Haskel pneumatic pump and methanol as the driving solvent. The role of methanol was to direct the packing material into the column as a very uniform dispersion of the particles. 
1,8-Nonadiyne was suspended into approximately $30 \mathrm{~mL} \mathrm{CCl}_{4} /$ methanol followed by 10 minutes of sonication. The $\mathrm{CCl}_{4}$ and silica's density are similar, and therefore choosing $\mathrm{CCl}_{4}$ allowed for an easier particle suspension. The slurry was then added to a reservoir tube, and the remainder of the tube was filled with HPLC grade methanol. The slurry was forced from the reservoir tube into the stainless steel tube under high pressure (6000 psi) in the presence of nitrogen gas. The stainless steel column was purchased from Alltech (Deerfiled, IL). The reservoir was made out of stainless steel for pressure durability. The entire packing procedure took about 15 minutes.

The column packing for 5-cyano-1-pentyne, 6-chloro-1-hexyne, and 4ethynylanaline was done by Stellar Phases, Inc. (Langhorne, PA). The column 5-cyano-1-pentyne with I.D.\# 11-110A was made by packing the synthesized material in to a $75 \mathrm{~mm}$ x $4.6 \mathrm{~mm}$ stainless steel column under $800 \mathrm{psi}$ at $1.0 \mathrm{~mL} / \mathrm{min}$ in $60 / 40$ ACN/ $\mathrm{H}_{2} \mathrm{O}$. The number of theoretical plates for this column is 3052. The newly made bonded phase 6-chloro-1-hexyne was packed into a $75 \mathrm{~mm}$ x $4.6 \mathrm{~mm}$ stainless steel column with the I.D.\# 11-110B under 700 psi and 60/ 40 ACN/ $\mathrm{H}_{2} \mathrm{O}$ solution. The number of theoretical plates for this column is 7077. The 4-ethynylanaline bonded phase was packed into a $75 \mathrm{~mm}$ x $4.6 \mathrm{~mm}$ i.d.column with I.D.\# 11-110C stainless steel column using $\sim 600 \mathrm{psi}$ and $60 / 40 \mathrm{ACN} / \mathrm{H}_{2} \mathrm{O}$. It was reported to have 3583 column theoretical plates with 1.26 asymmetry. 


\section{Instrumentation}

\section{Diffuse Reflectance Infrared Fourier Transform (DRIFT) spectroscopy}

DRIFT spectroscopy provides valuable qualitative information on the structure of the compound. The DRIFT characterization is obtained by verifying the characteristic bonds in the compound at a certain wavelength. Infrared radiation in the wavelength region of $4000-400 \mathrm{~cm}^{-1}$ is directed onto the powdered sample, and the diffusely reflected beam is collected into the spectrometer. The radiation absorption is characteristic of the vibrational modes of the functional groups in the compound. The absorptions are recorded by a detector and interpreted by a Fourier transform function in the form of spectra [28].

The equipment used for the qualitative analysis portion of this study was an ATI Mattson Infinity ${ }^{\text {TM }}$ series FTIR with a deuterated triglycine sulfate (DTGS) detector. Each sample was prepared separately by mixing the compound with $\mathrm{KBr}$ in 95:5 mg ratio. The mixture was kept in a heated oven $\left(110^{\circ} \mathrm{C}\right)$ for 24 hours. For the final preparation, the mixture sample was turned into a fine powder using a mortar and pestel for grinding. Each sample was put into a diffuse reflectance cup, a round hollow container $3 \mathrm{~mm}$ in diameter and $2 \mathrm{~mm}$ depth, and evened out on the surface by using a spatula. Prior to the actual run, the instrument was purged with $\mathrm{N}_{2}$ gas for approximately 20 minutes which removed unwanted peaks due to the presence of moisture and atmosphoric $\mathrm{CO}_{2}$. The infrared region at which the spectra were analyzed and recorded was $4000-400 \mathrm{~cm}^{-1}$. 


\section{Elemental (Carbon) Analysis}

It is important to evaluate the percentage of carbon on the surface of the newly synthesized stationary phases since it determines the surface coverage of the bonded phase. The surface coverage provides quantitative information about the organic groups on the surface of the silica hydride. The Berendsen and de Galan equation (3), shown below, provides the direct relationship between the two parameters, carbon percentage and surface coverage of the bonded phase.

$$
N=\frac{10^{6} P c}{1200 N_{c}-P_{c}(M-1)} \cdot \frac{1}{S}
$$

In the above equation, $P_{c}$ represents the difference in the percent of carbon between the bonded phase and silica hydride, obtainable through carbon analysis. $N_{c}$ is the number of carbons in the bonded organic group, obtained from the molecular formula of the substrate. $M$ is the molecular mass of the bonded molecule, and $\mathrm{S}$ is the Astrosil surface area in $\mathrm{m}^{2} / \mathrm{g}$, a value generally provided by the manufacturer $\left(350 \mathrm{~m}^{2} / \mathrm{g}\right.$ for this study) [29].

The carbon analyses of the samples were provided by Columbia Analytical Services, Inc. (Tuscon, AZ). The results will be discussed in the next section.

\section{Nuclear Magnetic Resonance (NMR) Spectroscopy}

One of the most powerful techniques in characterization of the compounds is nuclear magnetic resonance spectroscopy. NMR sepctroscopy is a special type of absorption spectrometry. The fundamental mechanism behind the technique relies on 
electromagnetic radiation absorption by the sample in an appropriate magnetic field. The absorption patterns of particular nuclei in the sample are characteristic of the compound structure [28].

For this particular study, samples were analyzed using solid-state NMR spectroscopy (CP-MAS ${ }^{29}$ Si NMR and ${ }^{13} \mathrm{C}$ NMR). Solid-state NMR provides detailed information in characterization of organic- inorganic (hybrid) materials. In addition to identifying the atomic environments, this technique can provide spatial details between various sites [30]. For samples dissolved in a solution (slurry), NMR spectra consist of very sharp peaks due to averaging of anisotropic NMR interactions by rapid random spinning. In solid state NMR, however, similar nuclei are relatively immobilized in many orientations with respect to the magnetic field, hence causing a peak broadening effect. The term chemical shift anisotropy (CSA) refers to orientation dependent interactions in solid-state NMR [31]. In order to mimic the sharp peaks generated from solution NMR in solid-state NMR, samples are spun at a high speed and at a specific angle, $55^{\circ}$, with respect to the magnetic field, called the magic angle. Magic angle spinning, MAS, stimulates the natural tumbling of the solution-phase molecules and results in the formation of sharper peaks. ${ }^{29} \mathrm{Si}$, with $4.7 \%$ natural abundance, allows a more sensitive environment (2.1 times) than ${ }^{13} \mathrm{C}$ but has a longer spin relaxation time [32]. The low abundance of certain nuclei could be the cause for their low sensitivity and therefore their inability to provide structural information when using solid-state NMR. To resolve this problem in solid-state NMR, the cross polarization (CP) technique is found to be helpful. In cross polarization the frequency of the less sensitive nucleus 
matches with the sensitive proton. This would increase the nucleus sensitivity significantly. Applying the $\mathrm{CP}$ technique decreases the amount of time taken to achieve a spectrum [31]. Single pulse ${ }^{29}$ Si magic angle spinning (MAS) provides information about Si atoms in the compound [33]. The peak broadening in solid-state NMR can provide useful information on chemistry, structure, and dynamics of the compound in the solid state [34].

The solid-state NMR for this study was done by the collaborative efforts of Professor Klaus Albert, et al. at Tubingen University in Germany. The spectra were obtained by utilizing MAS combined with CP. The characterization required approximately $150 \mathrm{mg}$ of each sample that was packed into a $\mathrm{ZrO}_{2}$ rotor and spun at an angle of $55^{\circ}$ with respect to the magnetic field with a CP contact time of $5 \mathrm{~ms}$.

\section{High Performance Liquid Chromatography (HPLC)}

The HPLC instrument used initially, and mainly for the 1,8-nonadiyene column, was a Shimadzu SPD-6A, as pictured in Figure 7. The system was equipped with a multi-channel interface, a Waters in-line degasser, and a Rheodyne Model 1726 manual injector with a $20 \mu \mathrm{L}$ injection loop and a UV detector. The instrument was connected to an HP AD converter, which in turn was connected to an HP Deskjet printer. The software for collecting the data on this equipment was Chemstation. The injections were done manually using a glass syringe. The $25.0 \mu \mathrm{L}$ glass syringe used in this experiment was a GASTIGHT® manufactured by Hamilton, Co. (Reno, NV). 
Prior to the runs, the instrument was set for a flow rate of $1.0 \mu \mathrm{L} / \mathrm{min}$, and the wavelength was set for $254 \mathrm{~nm}$. The 1,8-nonadiyne column was flushed with pure methanol for 30 minutes before the experiment. In order to achieve a wide range of hydrophilicity/ hydrophobicity in the mobile phase, HPLC grade acetonitrile and MilliQ water were mixed in a wide range of concentrations from a 10:90 to a 90:10 ratio. For mobile phases with an additive, 0.1 volumetric percent of formic acid was used in the mobile phases. All mobile phases were filtered using vacuum filtration. The samples were prepared by dissolving $1 \mathrm{mg}$ of the sample in $1 \mathrm{~mL}$ of acetonitrile: $\mathrm{H}_{2} \mathrm{O}$ (in a 50:50 ratio) with 0.1 volumetric percent of formic acid. All sample solutions were filtered with a $20 \mu \mathrm{m}$ nylon membrane.

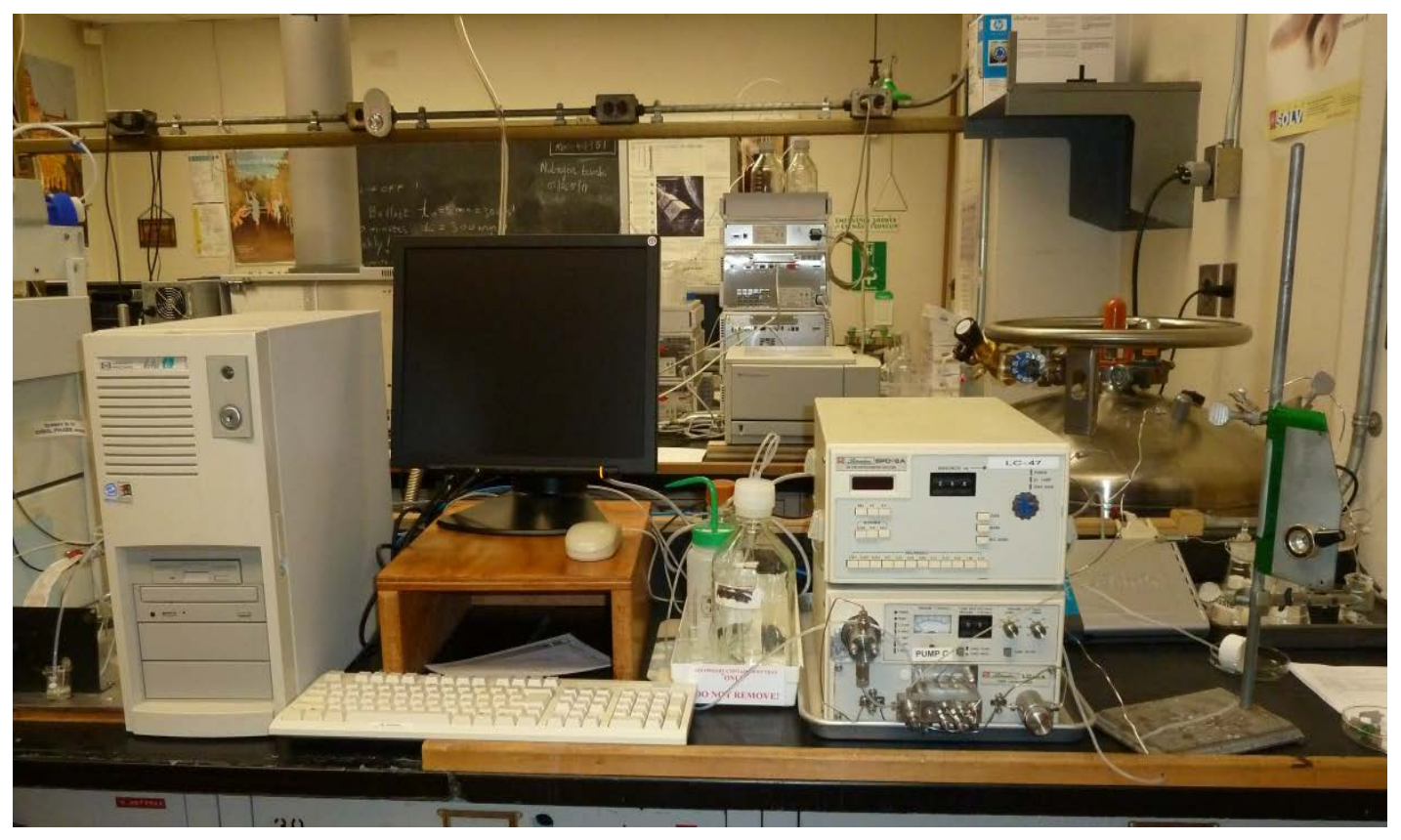

Figure 7. A view of the Shimadzu SPD-6A 
The liquid chromatographic studies of the rest of the columns for this research were performed using an HP Autosampler 1090 Series II. The instrument was equipped with a cabinet for a three solvent system, heated oven compartment, and an HP-IB interface board. The detector was a UV/Vis scan type or diode array detector (DAD). The instrument had a DR5 solvent delivery system which allowed the system to utilize from one to three solvent channels at any given time. This delivery system consisted of a solvent reservoir and a dual-syringe metering pump with a rotary valve, which allowed chromatographic performance in the isocratic mode (binary or ternary) as well as gradient elution. The HP 1090 had a solvent degassing nitrogen inlet line facilitating the removal of other gases dissolved in the solvents. The sampling system was auto-injection based with a 20 sample tray.

The machine was also equipped with a manual injector which facilitated a controlled single injection, whereas the auto injection increased the speed of the process as it can be programmed to perform up to 99 injections in a sequence. The sample injection valve was a six-port rotary valve designed for a high performance liquid chromatograph. The binary pump in HP 1090 had a very short delay time, comparable with the best pumps in the industry.

Upon an injection, an air actuator rotated the valve, and the solvent moved directly into the column as well as the injector needle which was connected to the syringe [34]. The nitrogen gas tank was from Praxair, UN 1066 N1 861090103. The datahandling device was HP-LC Chemstation software (Pascal series) with HP 3392A 
integrator, HP ThinkJet printer, HP PaintJet printer. The HP 1090 used for column characterizations and analyte retention studies is shown in Figure 8.

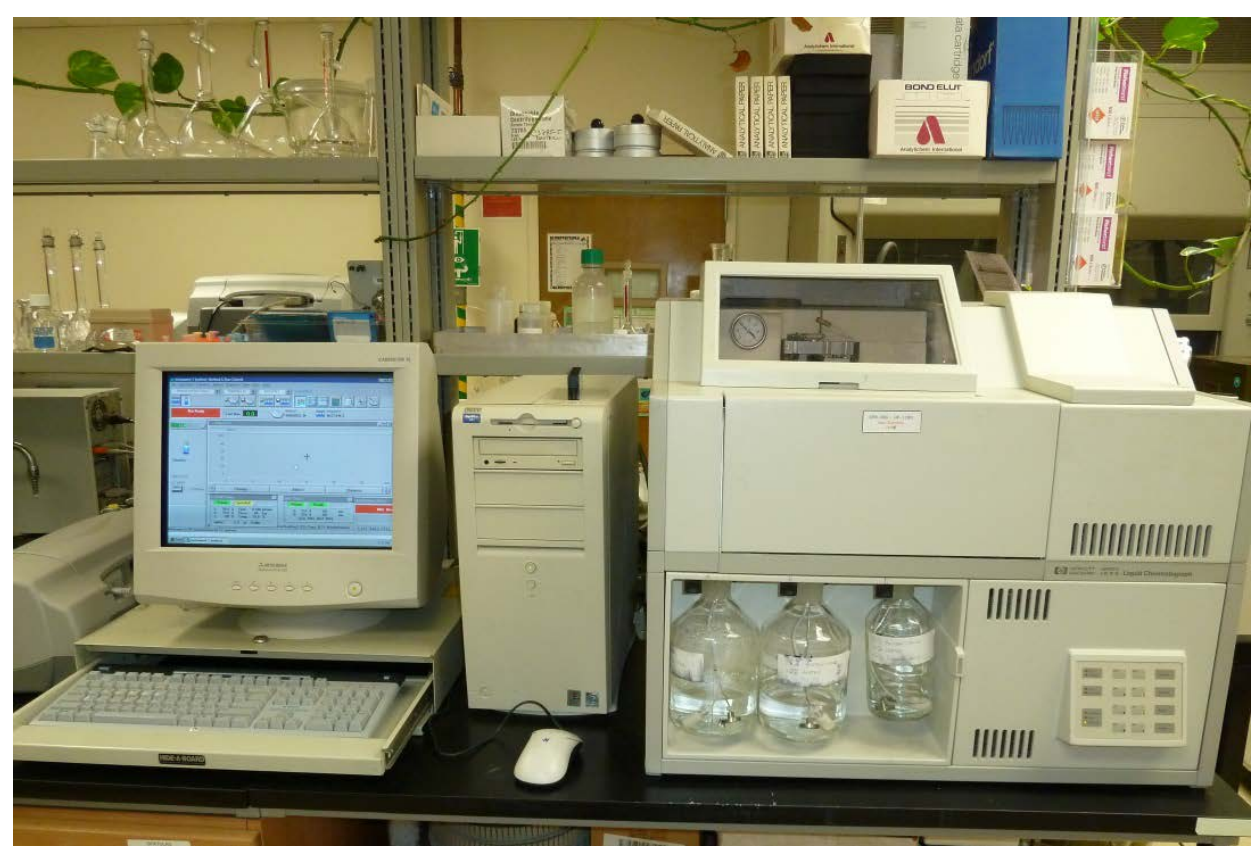

Figure 8. A view of the HP 1090 HPLC system

The mobile phase was vacuum filtered with a $0.2 \mu \mathrm{m}$ Nylon 66 membrane (Alltech Associates, Inc. Deerfield, IL) to enhance purity of the solvent before entering the instrument. The flow rate when using the autosampler was $0.5 \mathrm{~mL} / \mathrm{min}$.

The columns were flushed with $100 \%$ methanol for 1.0 hour as a means of preparation before the actual study. The instrument was set for two wavelengths of 214 nm and $254 \mathrm{~nm}$ for optimum detection of all analytes using the HP 1090 system. 


\section{RESULTS AND DISCUSSIONS}

\section{A. DRIFT Spectroscopic Characterization \\ 1. Silica hydride characterization}

The first step before performing multiple synthetic processes was to ensure the replacement of the $\mathrm{Si}-\mathrm{OH}$ bonds by $\mathrm{Si}-\mathrm{H}$ on the surface of silica via a silanization reaction. DRIFT spectroscopy confirmed the formation of the $\mathrm{Si}-\mathrm{H}$ bonds on the surface. As shown in Figure 9, DRIFT results showed a prominent peak at $2250 \mathrm{~cm}^{-1}$, a region which represents the $\mathrm{Si}-\mathrm{H}$ stretching vibration. The presence of a broad peak in the $3000-3750 \mathrm{~cm}^{-1}$ region can be explained by the presence of $\mathrm{H}_{2} \mathrm{O}$ molecules. The presence of a peak in near $3750 \mathrm{~cm}^{-1}$ region is due to $\mathrm{OH}$ stretching vibrations from the silanol groups. All other peaks pertain to the presence of siloxane backbone in the compound.

\section{Moiety surface characterization \\ i. 1,8-Nonadiyne}

Following the silanization reaction, hydrosilation of the 1,8-nonadiyne produced a bonded stationary phase on the surface of silica hydride. DRIFT spectroscopy results for 1,8-nonadiyne, as shown in Figure 10, confirmed the bonding of the organic moiety on the surface of the stationary phase. The significant size reduction in the $\mathrm{Si}-\mathrm{H}$ stretching bond region, $2200 \mathrm{~cm}^{-1}$, indicated that the surface was not exculsively populated by $\mathrm{Si}-\mathrm{H}$, hence confirming the presence of another group on the surface. 


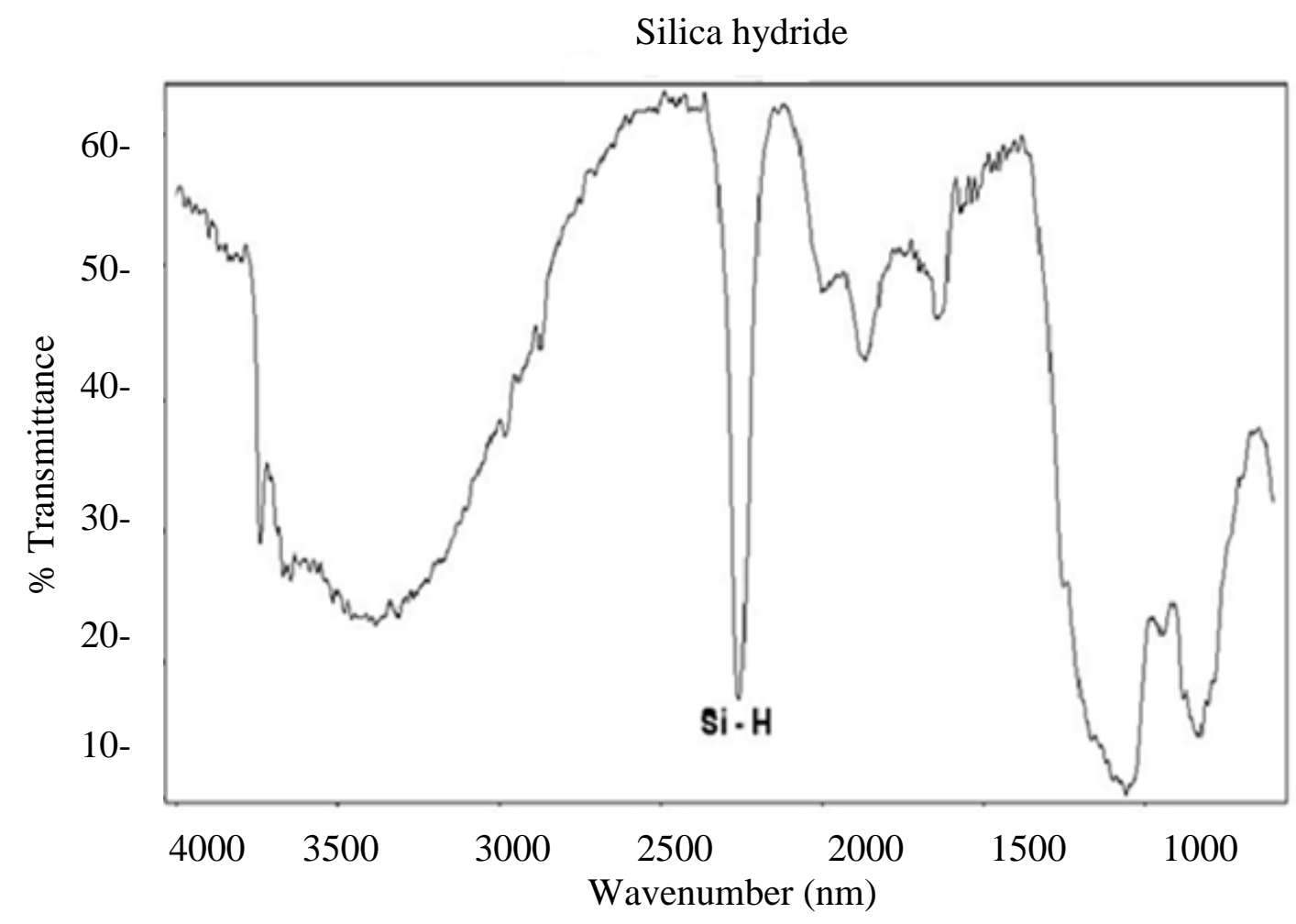

Figure 9. DRIFT spectrum for silica hydride identification

Aditionally, the presence of peaks at approximately $2800-3000 \mathrm{~cm}^{-1}$ region represents $\mathrm{C}-\mathrm{H}$ stretching bands which is indicative of an organic moeity, 1,8nonadiyne, for this experiment. Moreover, the sharp peak near $1700 \mathrm{~cm}^{-1}$ was indicative of the presence of a $\mathrm{C} \equiv \mathrm{C}$ bond which is consistent with the organic compound structure.

\section{ii. m-Diethynylbenzene}

The DRIFT spectrum for m-diethynylbenzene, as shown in Figure 11, showed a $\mathrm{Si}-\mathrm{H}$ peak size reduction. However, there were no other significant characteristic peaks that could verify either the attachment or significant surface coverage by the moiety. 
Further characterization techniques are needed to confirm or deny the hydrosilation of $m$ diethynylbenzene.

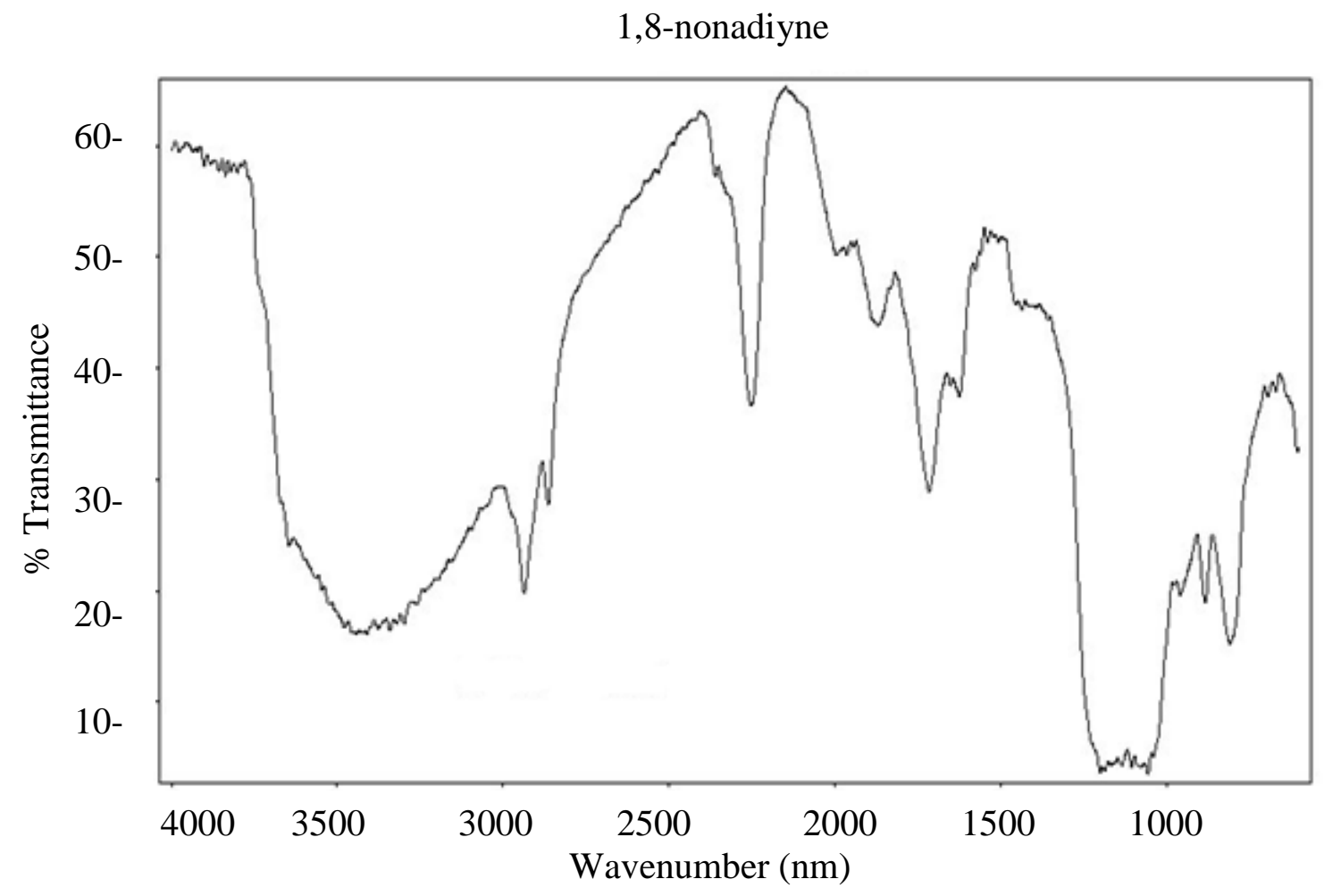

Figure 10. DRIFT spectrum of 1,8-nonadiyne bonded phase 


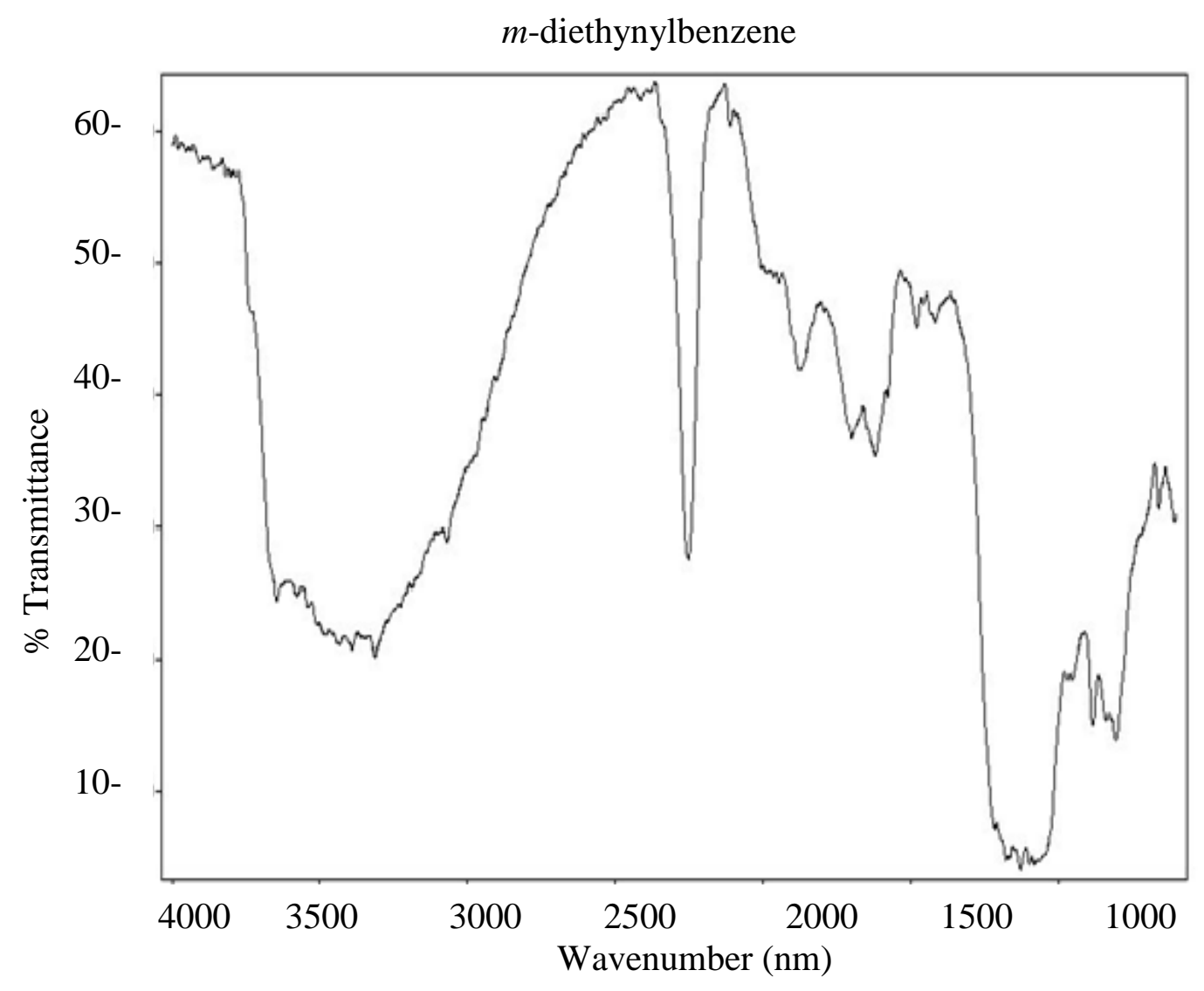

Figure 11. DRIFT spectrum for m-diethynylbenzene bonded phase

\section{iii. 5-Cyano-1-pentyne}

Figure 12 shows the DRIFT spectrum for the 5-cyano-1-pentyne stationary phase.

The insignificant size reduction in the $\mathrm{Si}-\mathrm{H}$ peak might be interpreted as low to zero surface coverage by the moiety. However, the presence of other characteristic peaks in the spectrum confirmed the formation of the 5-cyano-1-pentyne bonded phase. For instance, the presence of a peak at $1639 \mathrm{~cm}^{-1}$ could represent the $\mathrm{C}=\mathrm{C}$ stretching vibrations for the structures bonded to the silica hydride surface through the $\mathrm{C} \equiv \mathrm{N}$ functional group of 5-cyano-1-pentyne. This could also explain the unusually high 
intensity peak at $2240 \mathrm{~cm}^{-1}$ (the $\mathrm{Si}-\mathrm{H}$ region), (i.e., the peak was an overlap of the $\mathrm{Si}-\mathrm{H}$ and $\mathrm{C} \equiv \mathrm{N}$ stretching peaks).

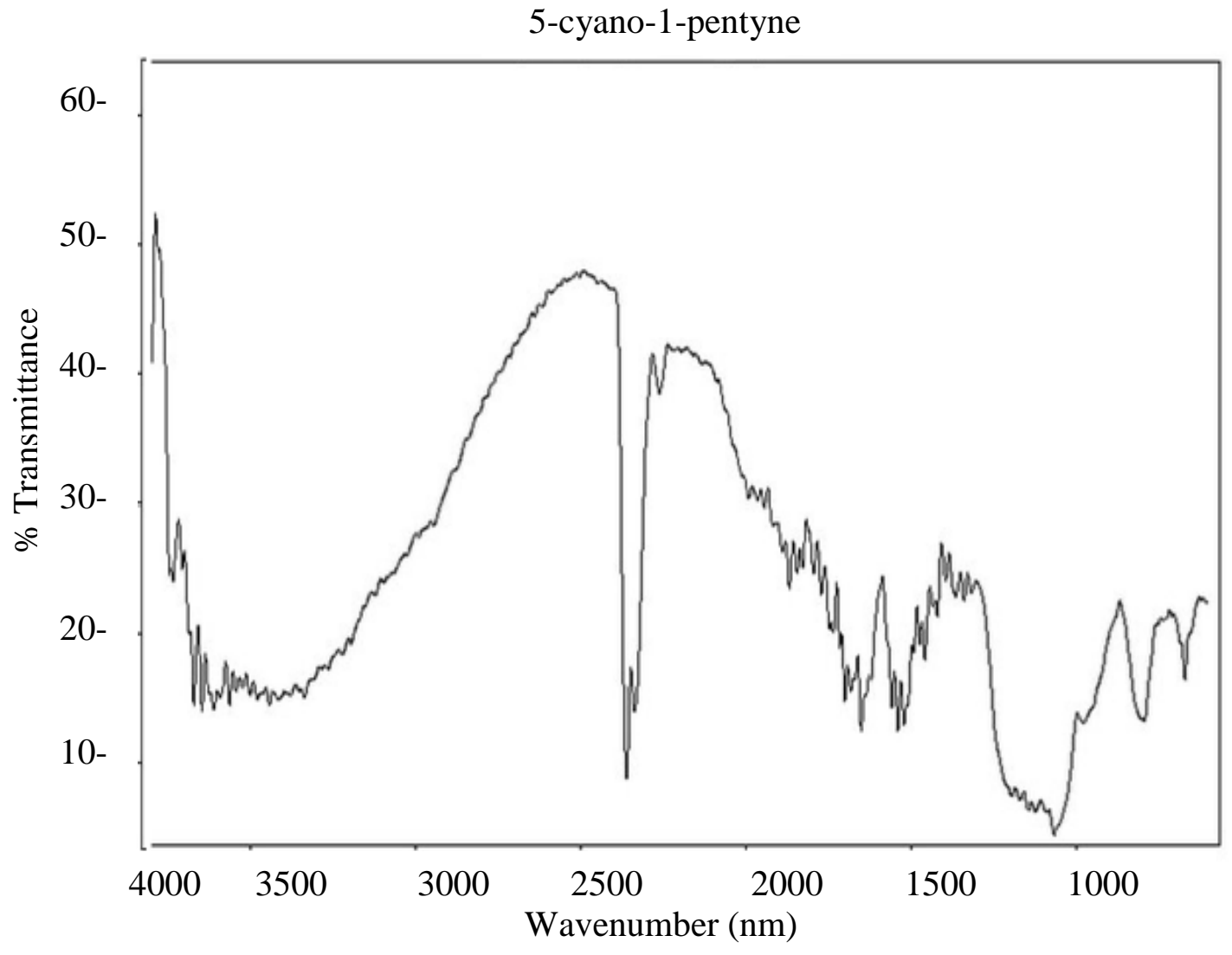

Figure 12. DRIFT spectrum of 5-cyano-1-pentyne bonded phase

\section{iv. 6-Chloro-1-hexyne}

The DRIFT spectrum for 6-chloro-1-hexyne confirmed the attachment of the moiety to the silica hydride surface. As shown in Figure 13, a significant $\mathrm{Si}-\mathrm{H}$ peak size reduction at $2240 \mathrm{~cm}^{-1}$ was indicative of the surface coverage by 6-chloro-1-hexyne. In addition, the presence of peaks at $2800-3000 \mathrm{~cm}^{-1}$ are the result of $\mathrm{C}-\mathrm{H}$ stretching 
bands of the organic moiety. Other smaller peaks in the spectrum could be due to the fundamental vibrations of organic functional groups and the siloxane backbone. A broad peak in the $3100-3700 \mathrm{~cm}^{-1}$ region is due to the presence of adsorbed water on the surface of the solid phase.

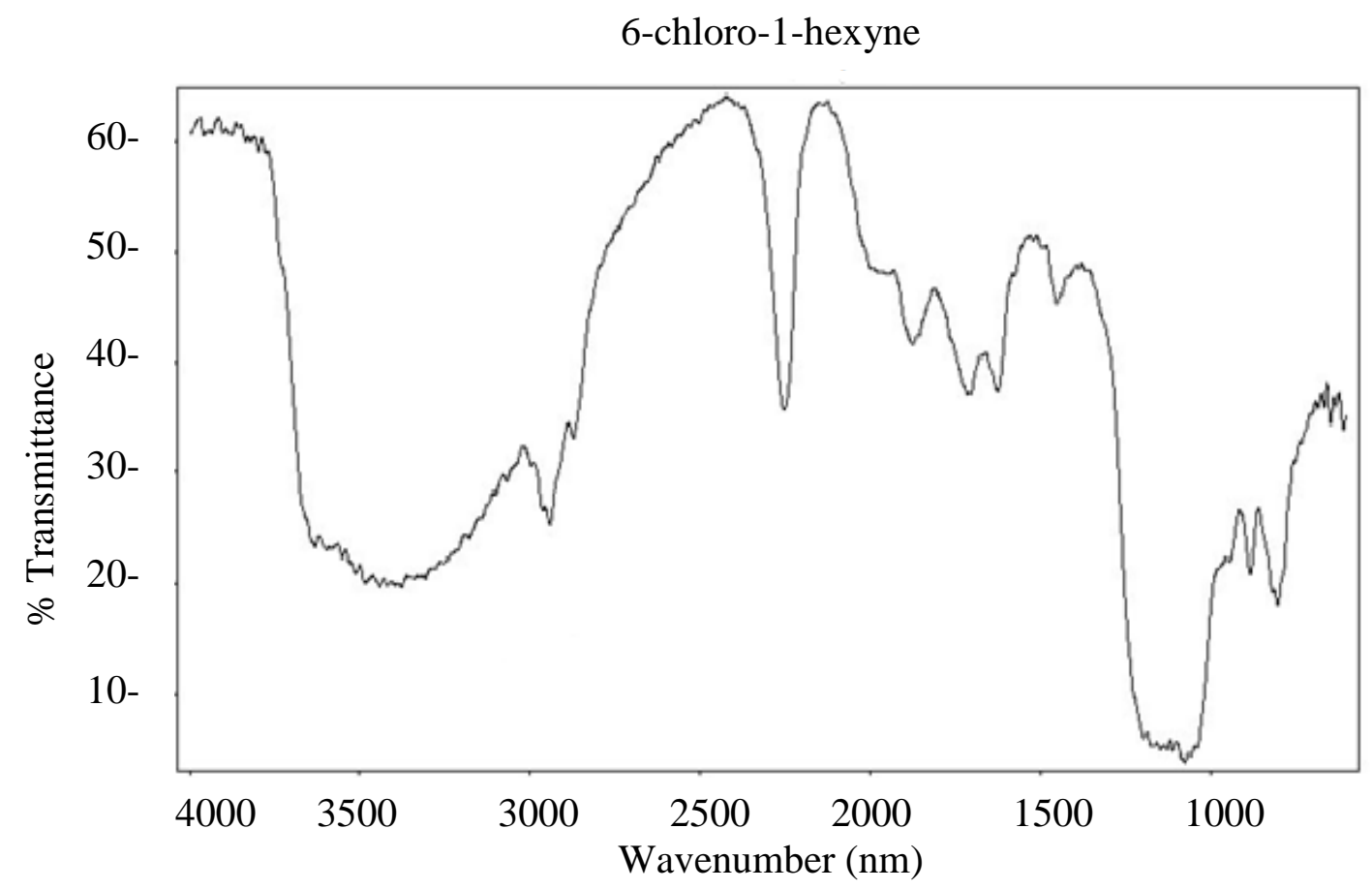

Figure 13. DRIFT spectrum of 6-chloro-1-hexyne bonded phase

\section{v. 4-Ethynylanaline}

The IR spectrum for 4-ethynylanaline, as shown in Figure 14, confirmed the bonding of the organic moiety to the silica hydride surface. The significant reduction in the $\mathrm{Si}-\mathrm{H}$ stretching peak intensity near the $2200 \mathrm{~cm}^{-1}$ region is indicative of successful bonding. Additionally, the aromatic $\mathrm{C}=\mathrm{C}$ stretching vibration appeared as a peak with 
medium intensity at $1500-1600 \mathrm{~cm}^{-1}$, another important characteristic peak to confirm the attachment to the silica hydride surface of 4-ethynylanaline.

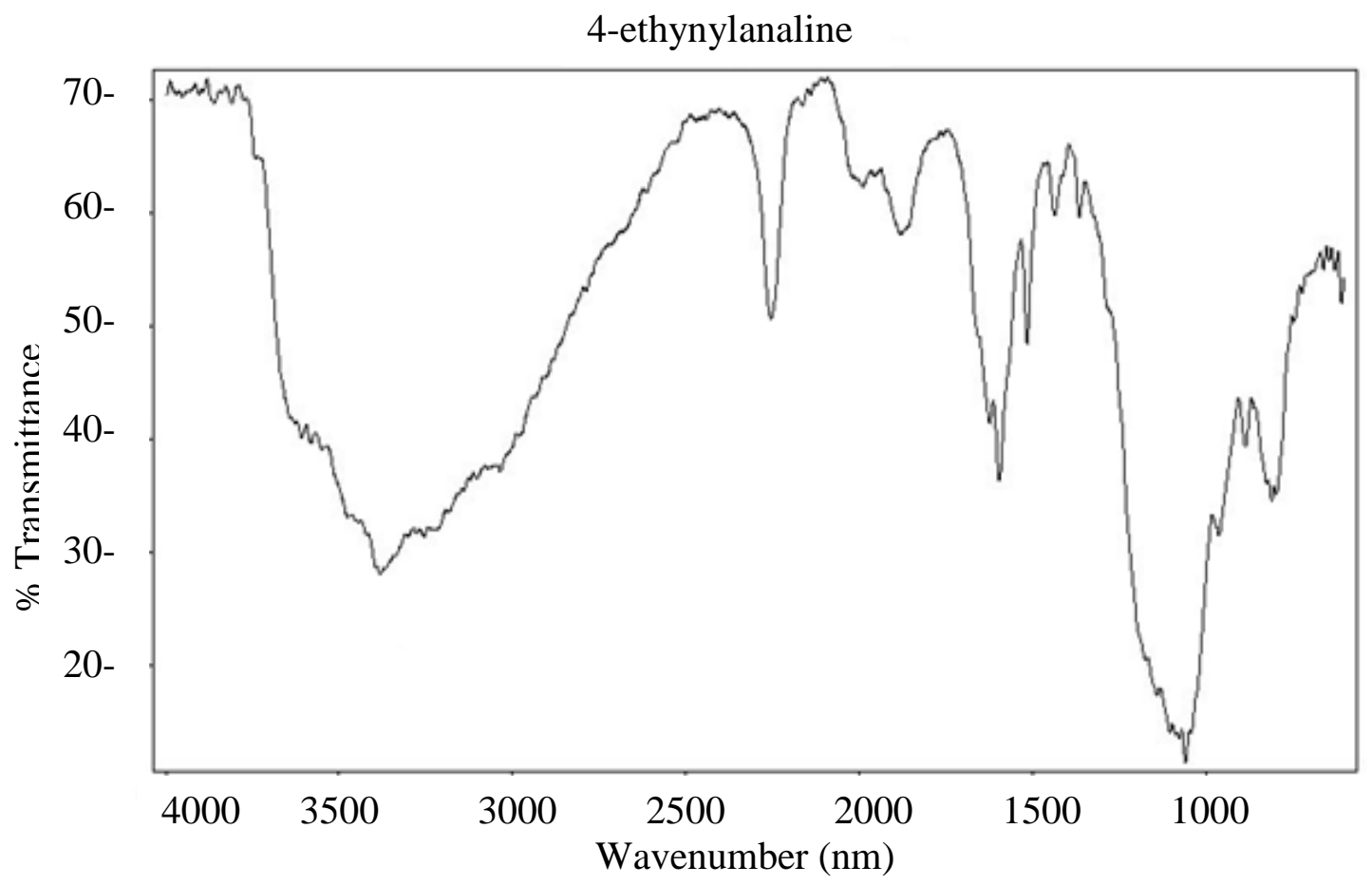

Figure 14. DRIFT spectrum of 4-ethynylanaline bonded phase

\section{B. Carbon analysis}

Quantitative analysis of organic moieties on the surface of silica hydride can be obtained through carbon analysis. Carbon analysis provides valuable information about the carbon load (percentage) and surface coverage of the organic moiety. Surface coverage plays an important role in the retention time, selectivity, and stability of the bonded phase. The physical structure of the substrate is a significant factor on the surface area of the packing material. In addition, the surface area is inversely related to the pore 
size of the packing material. Substrates with higher surface area provide greater capacity and longer analyte retention times. Conversely, substrates with lower surface area can be used for larger molecules when a faster equilibration time is desired. Surface coverage is calculated from the carbon load and surface area. Carbon load represents the amount of the bonded phase on the surface of silica. Higher carbon loads means greater column capacities and resolution.

The results obtained from the carbon analysis confirmed that $m$-dimethylbenzene did not show acceptable carbon percentage, and therefore it was removed from the rest of the study. Table 10 shows the carbon analysis values for all of the other four synthesized stationary phases. The surface coverage value for each bonded phase was calculated using the Berendsen and de Galan equation, as explained in the previous chapter.

Table 10. Carbon analysis of the newly synthesized bonded phases.

\begin{tabular}{|c|c|c|c|}
\hline Compound & $\begin{array}{c}\text { Bulk } \\
\text { carbon } \\
(\%)\end{array}$ & $\begin{array}{c}\text { Surface } \\
\text { area } \\
\left(\mathrm{g} / \mathrm{m}^{2}\right)\end{array}$ & 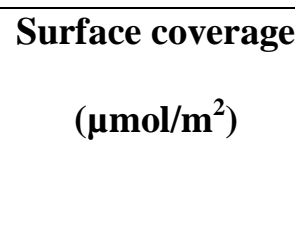 \\
\hline 1,8-Nonadiyne & 7.480 & 350.00 & 2.157 \\
\hline 5-Cyano-1-pentyne & 1.520 & 350.00 & 0.615 \\
\hline 6-Chloro-1-hexyne & 6.180 & 350.00 & 3.034 \\
\hline 4-Ethynylanaline & 12.750 & 350.00 & 4.487 \\
\hline
\end{tabular}




\section{NMR Characterization}

\section{Solid-state NMR (SS-NMR): Characterization with ${ }^{29} \mathrm{Si} \mathrm{CP} / \mathrm{MAS}$ and ${ }^{13} \mathrm{C}$ CP/MAS NMR}

\section{i. 1,8-Nonadiyene}

Figure 15 is the ${ }^{29} \mathrm{Si} \mathrm{CP} / \mathrm{MAS}$ NMR spectrum for the 1,8-nonadiyne bonded phase. The main characteristic in spectra like this one is the $\mathrm{Si}-\mathrm{H}$ peak size at $85 \mathrm{ppm}$. The presence of an organic moiety on the surface of a silica hydride base would cause a decrease in the intensity of the peak at 85 ppm when compared with that of a non-bonded stationary phase. Naturally, the more populated the surface, the less free $\mathrm{Si}-\mathrm{H}$ on the stationary phase and the smaller the 85 ppm peak would be.

The $\mathrm{Si}-\mathrm{H}$ peak size reduction at 85 ppm observed for 5-cyano-1-pentyne is shown in Figure 17. The same effect is observed for 6-chloro-1-hexyne and 4ethynylanaline, as shown in Figures 19 and 21, respectively. 

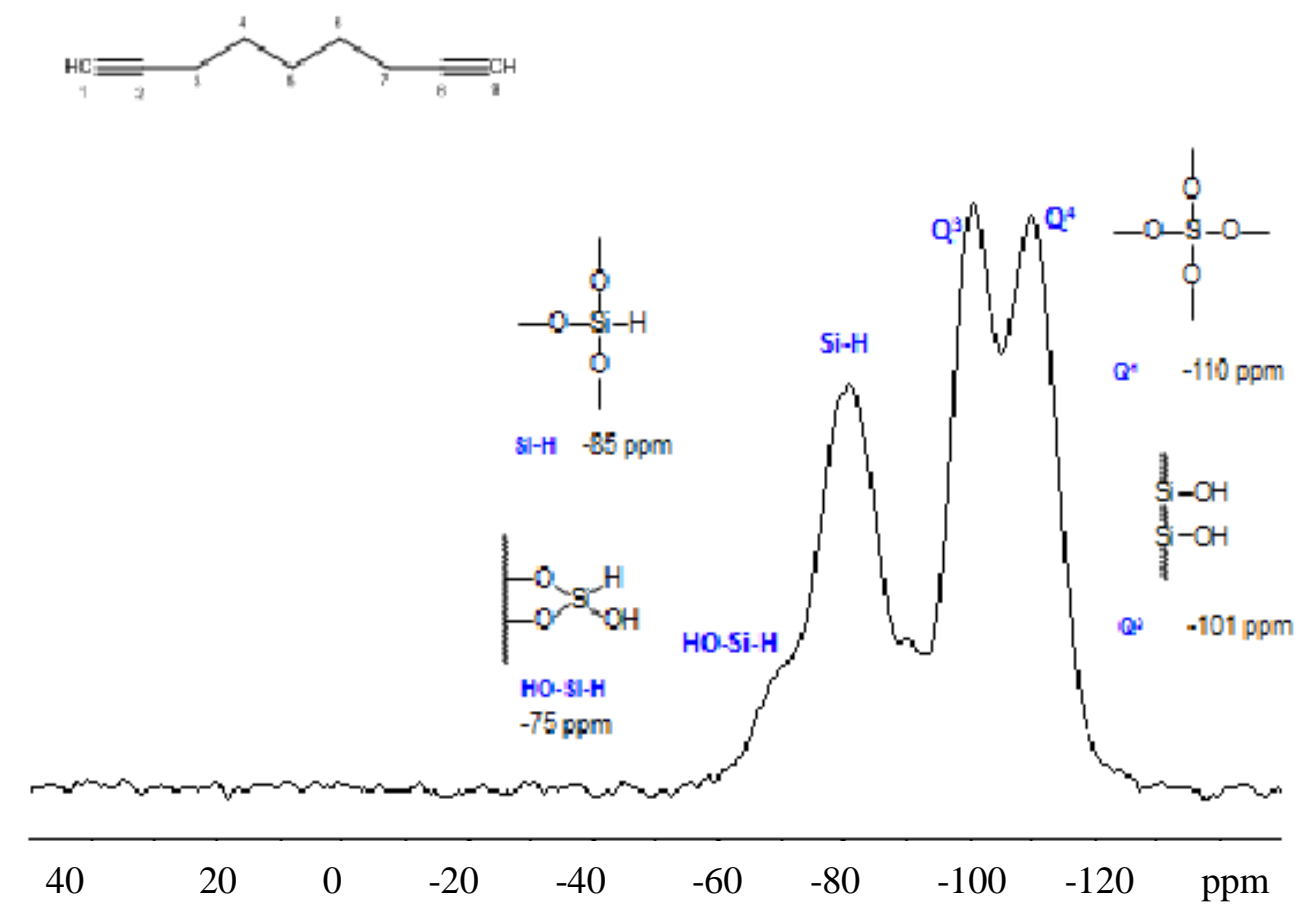

Figure 15. ${ }^{2 y} \mathrm{Si} \mathrm{CP} / \mathrm{MAS}$ NMR spectrum for the 1,8-nonadiyne stationary phase

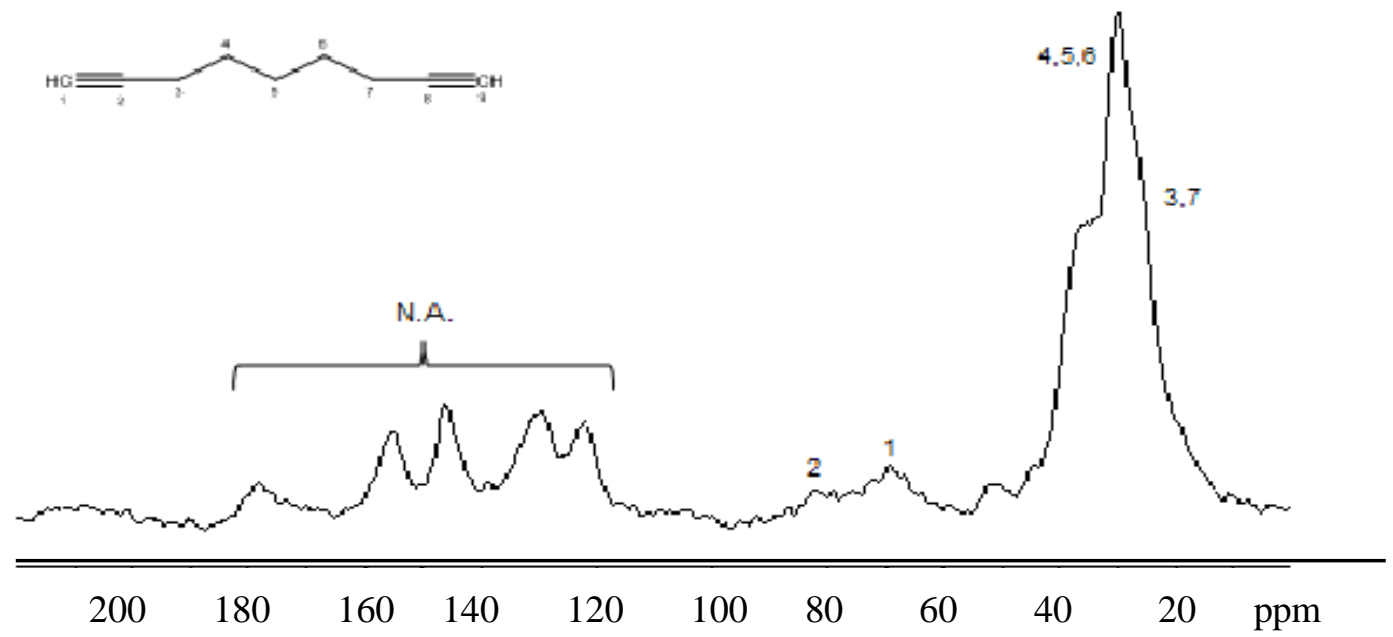

Figure 16. ${ }^{13} \mathrm{C} \mathrm{CP} / \mathrm{MAS}$ NMR spectrum for the 1,8-nonadiyne stationary phase 
The ${ }^{13} \mathrm{C}$ CP-MAS NMR spectrum is another means to verify the bonding of the organic moiety by hydrosilation. Figure 16 shows the ${ }^{13} \mathrm{C}$ CP-MAS NMR spectrum of the 1,8-nonadiyne bonded phase. The appearance of peaks in the low ppm region (29.5 $35.5 \mathrm{ppm}$ ) is characteristic of the saturated alkane 1,8-nonadiyne, (i.e., carbons 3, 4, 5, 6, and 7 specifically). The non-bonded terminal alkynes $(1,2)$ appeared further up in the spectrum (69.4- 85.0 ppm). The ${ }^{13} \mathrm{C}$ CP-MAS-NMR showed an interesting feature about another possible form of attachment of 1,8-nonadiyne to the surface. The presence of certain peaks at higher ppm (121.9 - $178.5 \mathrm{ppm})$ raises the possibility of formation of a bridge-like structure, where 1,8-nonadiyne could bond to the surface from both ends (terminal alkynes), hence the formation of alkenes in the structure of the bridge.

\section{ii. 5-Cyano-1-pentyne}

A significant decrease in the $\mathrm{Si}-\mathrm{H}$ peak intensity at $85 \mathrm{ppm}$ in ${ }^{29} \mathrm{Si} \mathrm{CP}-\mathrm{MAS}$ $\mathrm{NMR}$, as well as the presence of a sharp $\mathrm{C} \equiv \mathrm{N}$ peak in the high-chemical-shift region (120 ppm) of ${ }^{13} \mathrm{C}$ CP-MAS NMR, confirmed the formation of the 5-cyano-1-pentyne bonded stationary phase. Figures 17 and 18 represent the ${ }^{29} \mathrm{Si}$ and ${ }^{13} \mathrm{C}$ CP-MAS NMR spectra for the cyano bonded moiety, respectively. 


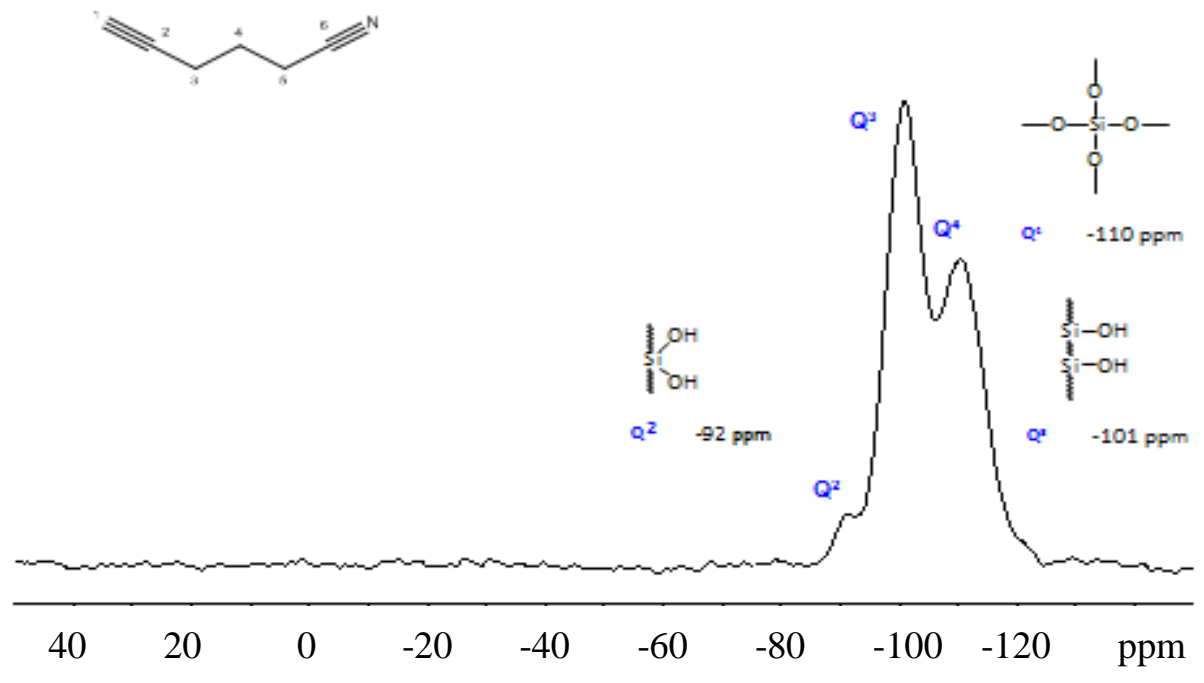

Figure 17. ${ }^{29} \mathrm{Si} \mathrm{CP} / \mathrm{MAS}$ NMR spectrum for the 5-cyano-1-pentyne stationary phase

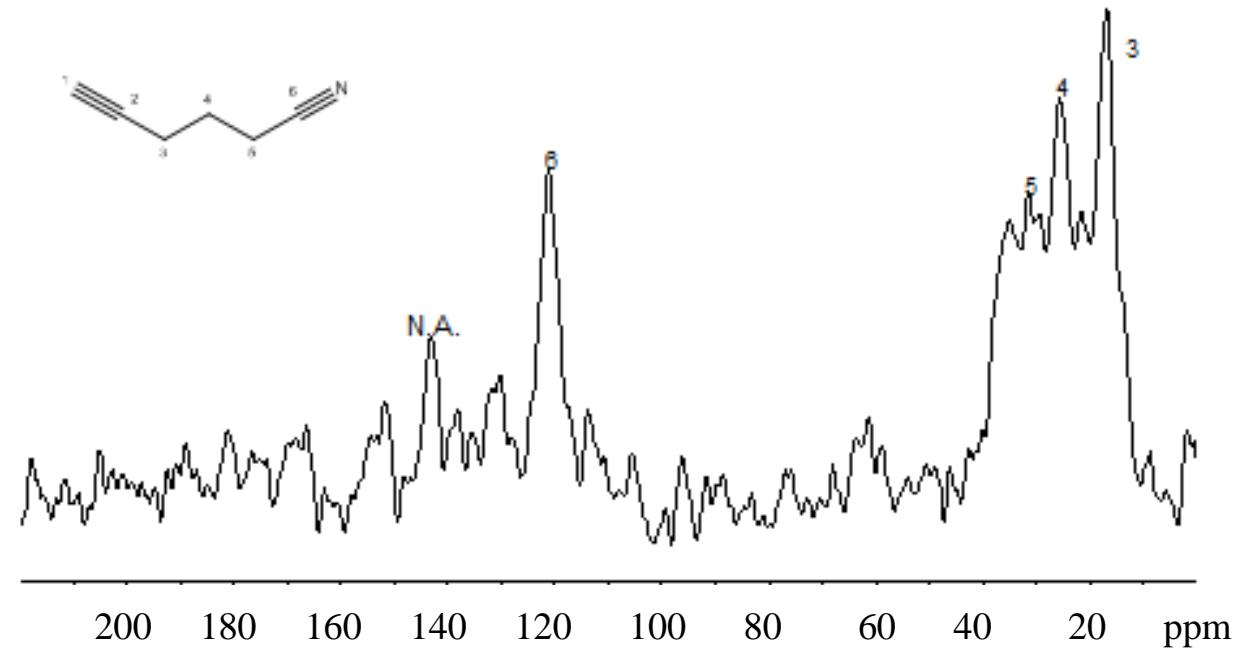

Figure 18. ${ }^{13} \mathrm{C} \mathrm{CP} / \mathrm{MAS}$ NMR spectrum for the 5-cyano-1-pentyne stationary phase 


\section{iii. 6-Chloro-1-hexyne}

The ${ }^{29} \mathrm{Si}$ and ${ }^{13} \mathrm{C} \mathrm{CP} / \mathrm{MAS}$ NMR for the 6-chloro-1-hexyne stationary bonded phase confirmed the attachment of the moiety to the surface of silica hydride. Figures 19 and 20 are the ${ }^{29} \mathrm{Si}$ and ${ }^{13} \mathrm{C} \mathrm{CP} / \mathrm{MAS}$ NMR spectra, respectively. The lower intensity peak at -85 ppm in ${ }^{29} \mathrm{Si} \mathrm{CP/} \mathrm{MAS} \mathrm{NMR} \mathrm{is} \mathrm{indicative} \mathrm{of} \mathrm{bonding} \mathrm{of} \mathrm{the} \mathrm{organic} \mathrm{moiety} \mathrm{to}$ the silica hydride surface. The ${ }^{13} \mathrm{C} \mathrm{CP} / \mathrm{MAS}$ NMR spectrum shows the presence of peaks at the high-chemical-shift region between 122.2 to $155.2 \mathrm{ppm}$ that represents the olefinic carbons in the 6-chloro-1-hexyne molecule.
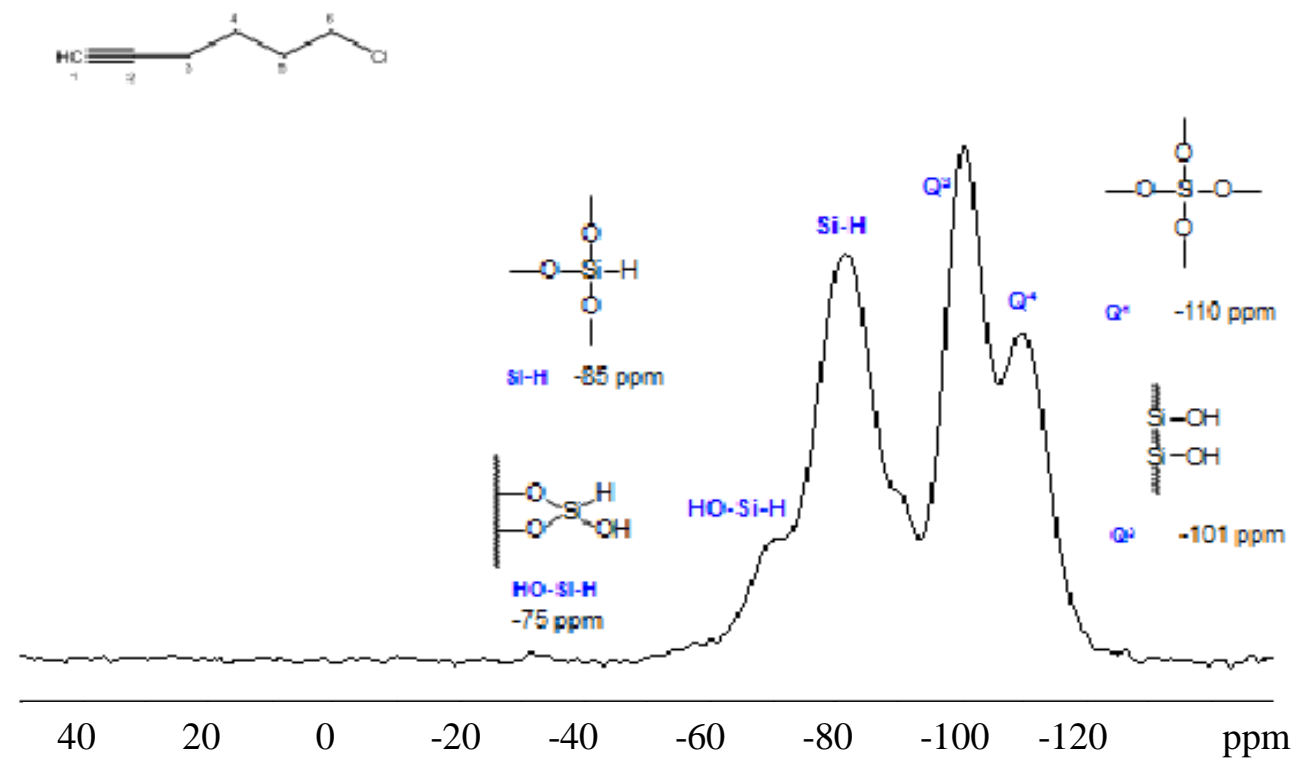

Figure 19. ${ }^{29} \mathrm{Si} \mathrm{CP} / \mathrm{MAS}$ NMR spectrum for the 6-chloro-1-hexyne stationary phase 


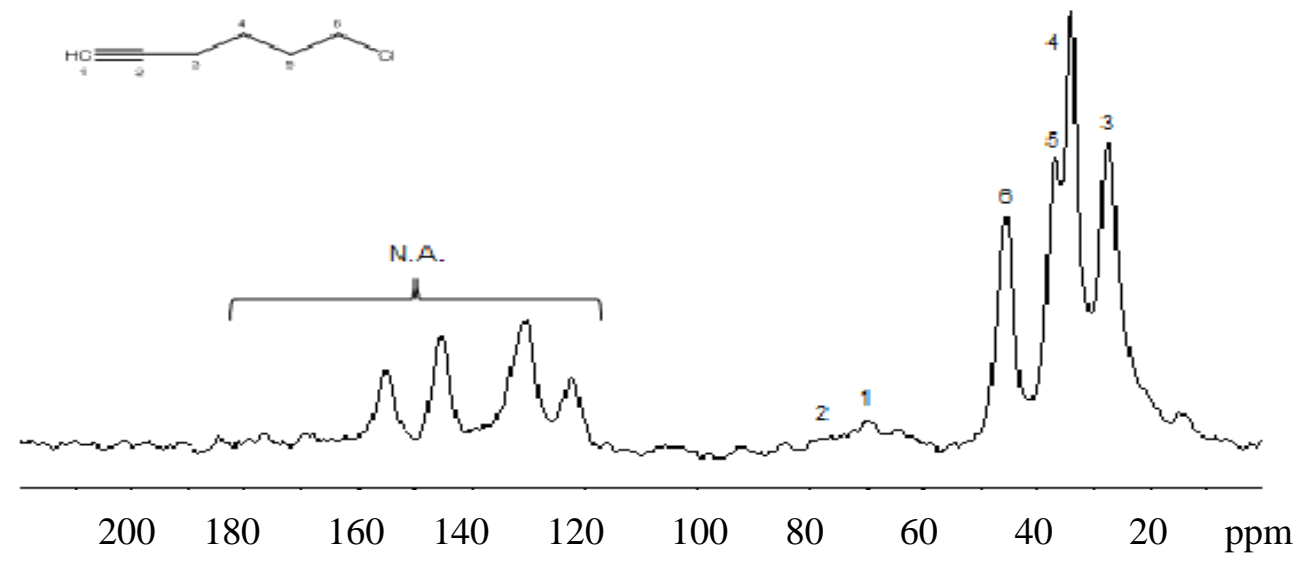

Figure 20. ${ }^{13} \mathrm{C} \mathrm{CP/} \mathrm{MAS} \mathrm{NMR} \mathrm{spectrum} \mathrm{for} \mathrm{the} \mathrm{6-chloro-1-hexyne} \mathrm{stationary} \mathrm{phase}$

\section{iv. 4-Ethynylanaline}

The peak size decrease at $85 \mathrm{ppm}$ in ${ }^{29} \mathrm{Si} \mathrm{CP} / \mathrm{MAS}$ NMR spectrum for 4-ethynylanaline confirmed the bonding of the organic moiety on the silica hydride surface. The presence of characteristic peaks, i.e. aromatic carbons between 116.3 to 154.3 ppm, as well as unsaturated carbons peaks at the high-chemical-shift region of $200.6 \mathrm{ppm}$ in ${ }^{13} \mathrm{C} \mathrm{CP} / \mathrm{MAS}$ NMR, validated the attachment of the organic moiety onto the silica hydride surface. 


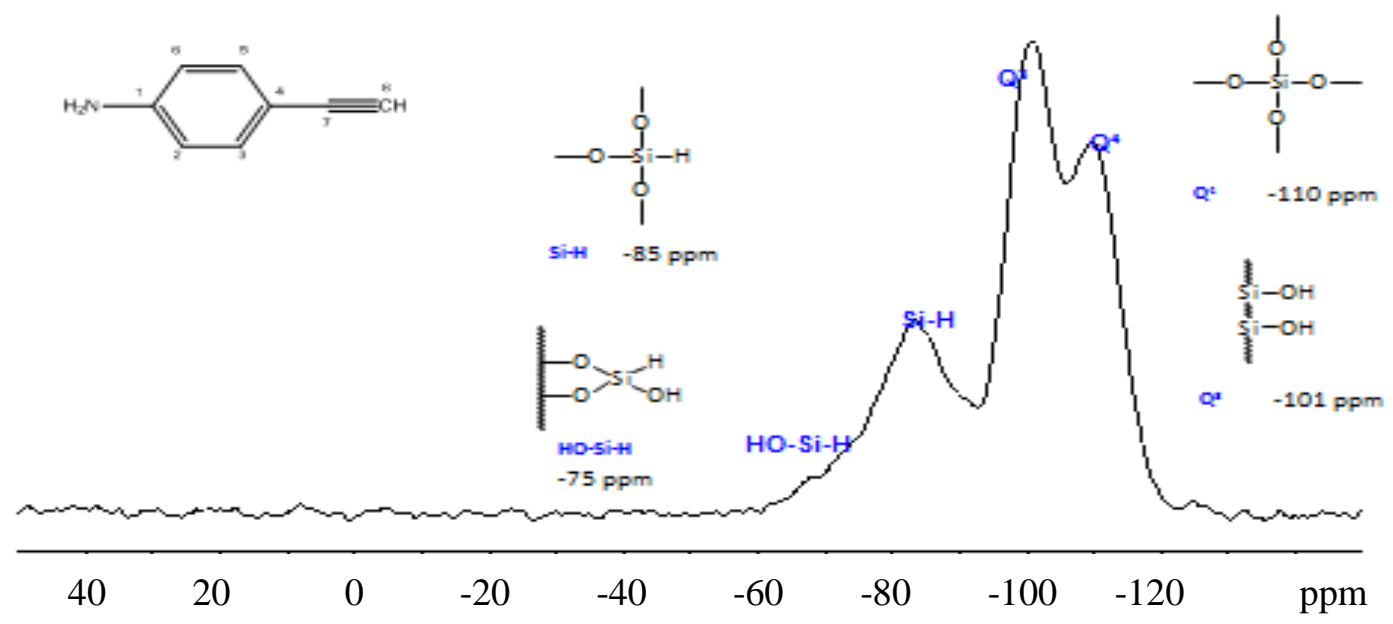

Figure 21. ${ }^{29} \mathrm{Si} \mathrm{CP} / \mathrm{MAS}$ NMR spectrum for the 4-ethynylanaline stationary phase

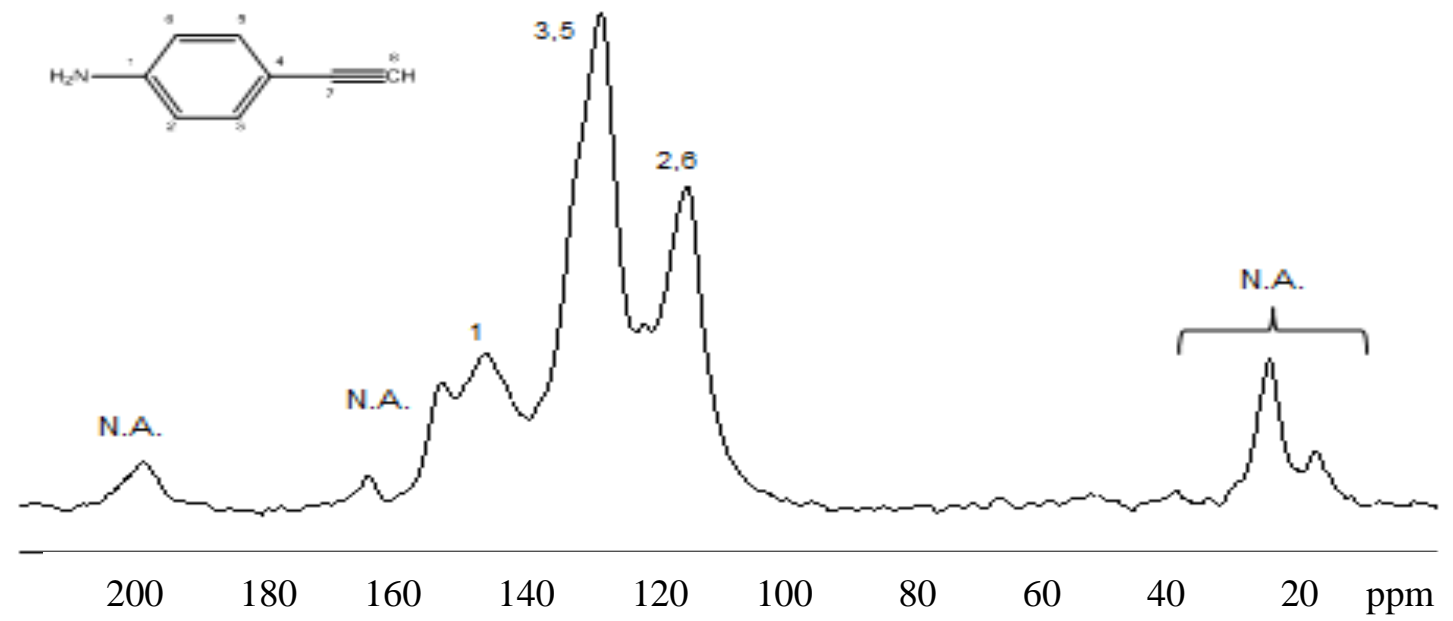

Figure 22. ${ }^{13} \mathrm{C} \mathrm{CP} / \mathrm{MAS}$ NMR spectrum for the 4-ethynylanaline stationary phase 


\section{HPLC Chromatographic Evaluation of the Columns}

To evaluate the newly synthesized bonded stationary phases and obtain a retention map for each column, a series of chromatographic evaluations was performed on the columns. To achieve this goal, a wide selection of analytes ranging from those that exhibit ANP behavior (hydrophilic molecules such as amino acids, nucleic bases, neurotransmitters) to analytes with RP characteristics (hydrophobic compounds such as steroids and polycyclic aromatic hydrocarbons) was chosen for retention behavior studies. The binary mobile phase for these evaluations was HPLC grade acetonitrile (ACN)/ MilliQ DI $\mathrm{H}_{2} \mathrm{O}$. The ratio of the two components of the mobile phase was altered at each step in an isocratic fashion.

\section{1,8-Nonadiyne}

The column was first washed with methanol: water $(50: 50)$ to remove unwanted peaks produced by impurities. The flow rate was optimized to $0.5 \mathrm{~mL} / \mathrm{min}$, and the injection volume was set to $0.2 \mu \mathrm{L}$ for all the samples.

\section{i. Hydrophilic analytes}

The retention behaviors of the analytes that exhibit ANP behavior used for the evaluation of 1,8-nonadiyene are listed in the following tables: 11, 12, 13. The dissolving solvent was (50:50) acetonitrile : DI $\mathrm{H}_{2} \mathrm{O}$ with $0.1 \%$ by volume of formic acid. All solid samples were weighed exactly to maintain the $1.0 \mathrm{mg}$ of analyte per $1.0 \mathrm{~mL}$ of solvent

ratio. However, there were several samples with which the column experienced 
overloading, an unwanted phenomenon produced by an excessive concentration of the solute in the solution. Overloading the column could result in peak shape distortions. To solve this problem the analytes were diluted at least once, and in some cases twice, by a (10:90) solute to solvent ratio. The chromatographic runs began in the RP mode which means a high percentage of water at the beginning followed by a gradual increase in the concentration of the less polar component (ACN). The retentions times for the two amino acid analytes are listed in Table 11.

Table 11. Retention times of the hydrophilic amino acids using the 1,8-nonadiyne column.

\begin{tabular}{|c|c|c|c|}
\hline \multicolumn{2}{|c|}{ Concentration of the } & \multicolumn{2}{c|}{ (min) } \\
mobile phase (\%) & Phenylglycine & 3.9510 \\
\hline Acetonitrile & DI water & 2.9000 & 3.6250 \\
\hline 10 & 90 & 2.8290 & 3.2900 \\
\hline 20 & 80 & 2.6969 & 2.9850 \\
\hline 30 & 70 & 2.3200 & 2.9170 \\
\hline 40 & 60 & 2.1930 & 3.0690 \\
\hline 50 & 50 & 2.2840 & 3.9150 \\
\hline 60 & 40 & 2.3430 & 6.1870 \\
\hline 70 & 30 & 2.7180 & $*$ \\
\hline 80 & 20 & $*$ & \\
\hline 90 & 10 & & \\
\hline
\end{tabular}

* peak was not observed 
Figure 23 shows the retention maps for the hydrophilic amino acids, phenylalanine and phenylglycine ( $\bullet$ and $\boldsymbol{\Lambda}$, respectively). According to these maps, as the polarity of the mobile phase decreases, i.e. from $50 \%$ to $80 \% \mathrm{ACN}$, the retention time for polar compounds increases (typical ANP behavior). Inversely, the retention time of the compounds decreases when the mobile phase becomes more hydrophilic, (i.e., above $50 \% \mathrm{H}_{2} \mathrm{O}$, showing the RP behavior). The ANP behavior of the analytes is a result of various interactions between the analyte and polar moieties of the stationary phase. The pivotal point for the column, (i.e., switching from RP mode to ANP) is around 50\% ACN. Therefore, if the compound carries both hydrophilic and hydrophobic properties, it will be retained on a silica hydride column. Both phenylalanine and phenylglycine are polar and have ANP behavior on the column in the presence of a relatively non-polar mobile phase.

Theoretically, for a column with dual properties (RP and ANP) and a mobile phase composed of binary solvent system (polar and non-polar) the column behaves in RP mode at lower concentrations of non-polar solvent and vice versa. The behaviors were observed for both phenylalanine and phenylglycine amino acids when the \%ACN shifts from low to high, forming a curve with a U-shape property.

The next group of hydrophilic compounds to be analyzed under RP/ ANP conditions was the two nucleobases, adenine and guanine. According to Table 12, both guanine and adenine have reasonable retention and show noticeable ANP behavior on this column. The retention for adenine is more significant than that of guanine. On a side note, guanine was very difficult to dissolve in this diluent. In order to dissolve this 
compound, an additional amount of water, followed by warming the solution, was required. The actual retention behaviors of adenine and guanine ( $\boldsymbol{\Delta}$ and $><$, respectively) with 1,8-nonadiyne column are shown in Figure 24.

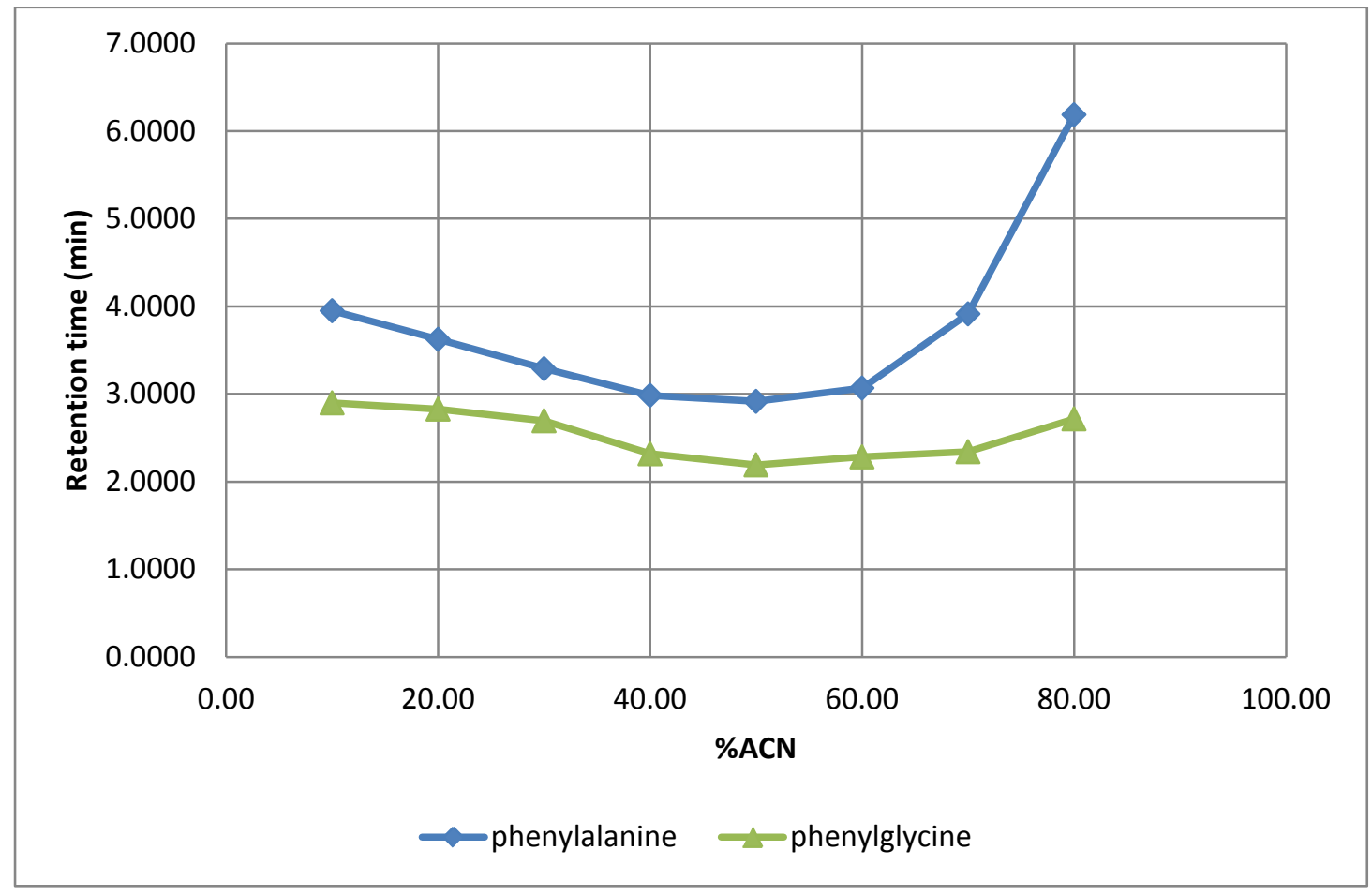

Figure 23. Retention map of the amino acids, phenylalanine and phenylglycine, using the 1,8-nonadiyne column 
Table 12. Retention times of the hydrophilic nucleobases using the 1,8-nonadiyne column.

\begin{tabular}{|c|c|c|c|}
\hline \multicolumn{2}{|c|}{$\begin{array}{l}\text { Concentration of the } \\
\text { mobile phase (\%) }\end{array}$} & \multicolumn{2}{|c|}{$\begin{array}{l}\text { Retention time } \\
\text { (min) }\end{array}$} \\
\hline Acetonitrile & DI water & Adenine & Guanine \\
\hline 10 & 90 & * & $*$ \\
\hline 20 & 80 & $*$ & 5.0410 \\
\hline 30 & 70 & 12.2460 & 3.9740 \\
\hline 40 & 60 & 8.2220 & 3.4120 \\
\hline 50 & 50 & 5.8610 & 3.6345 \\
\hline 60 & 40 & 6.0850 & 3.9070 \\
\hline 70 & 30 & 8.3130 & 4.2811 \\
\hline 80 & 20 & 16.1518 & 5.8140 \\
\hline 90 & 10 & * & $*$ \\
\hline
\end{tabular}

* peak was not observed 


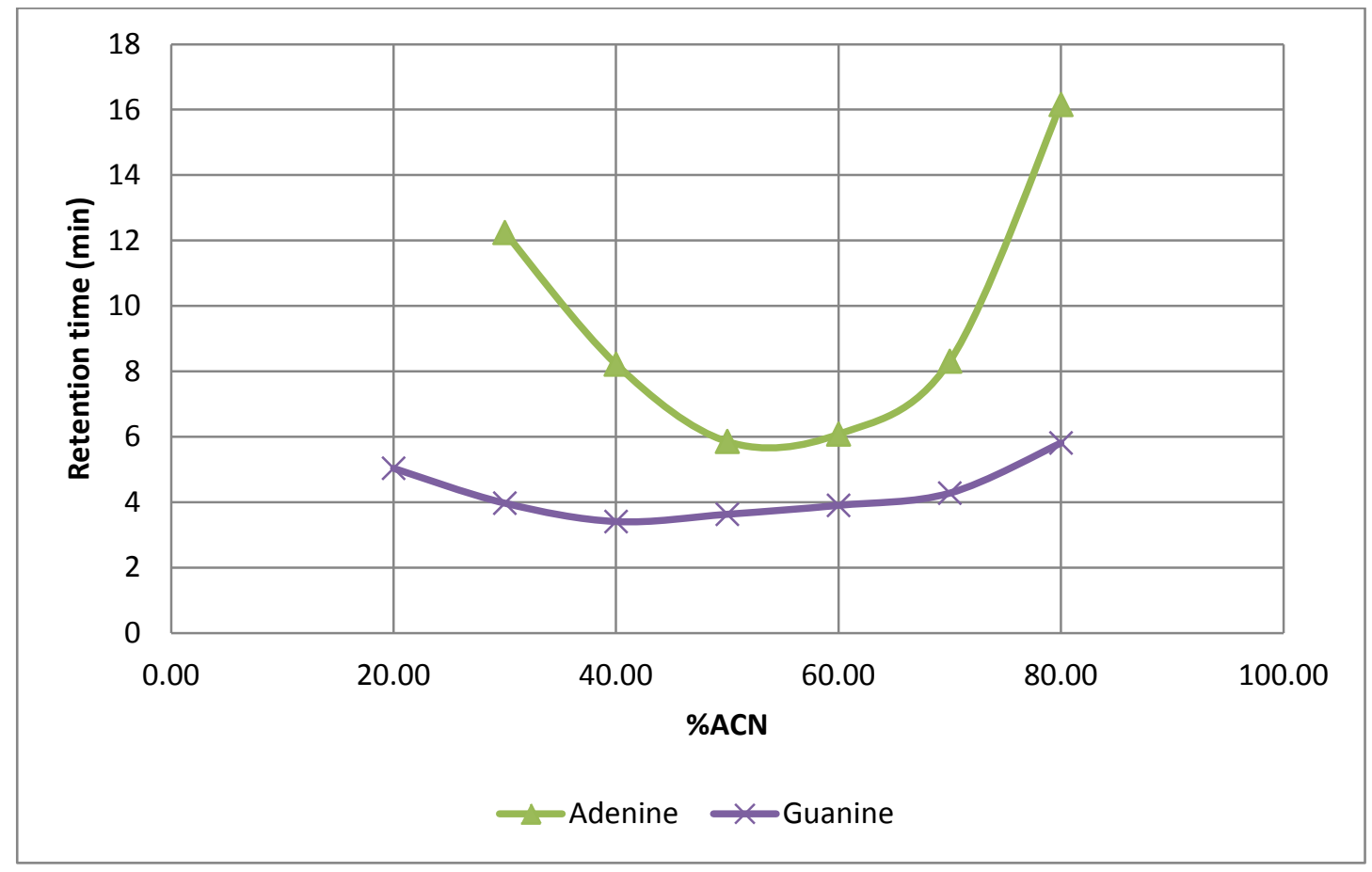

Figure 24. Retention map of the nucleobases, adenine and guanine, using the 1,8-nonadiyne column

According to the retention maps for the two nucleobases, adenine and guanine, the 1,8-nonadiyne bonded stationary phase shows RP and ANP retention for these polar compounds.

Neurotransmitters are the next group of polar analytes used for the characterization of the 1,8-nonadiyne column. Epinephrine, norepinephrine, and dopamine all show some ANP behavior on the column under the less-polar conditions (higher concentrations of ACN) of the mobile phase. Table 13 shows the retention times of these analytes. 
Table 13. Retention times of the hydrophilic neurotransmitters using the 1,8-nonadiyne column.

\begin{tabular}{|c|c|c|c|c|}
\hline \multicolumn{2}{|c|}{ Concentration of the } & \multicolumn{3}{c|}{ (min) } \\
mobile phase (\%) & & Norepinephrine & Dopamine \\
\hline Acetonitrile & DI water & Epinephrine & 2.3800 & 2.8490 \\
\hline 10 & 90 & 2.8290 & 2.3560 & 2.7320 \\
\hline 20 & 80 & 2.7330 & 2.3070 & 2.4120 \\
\hline 30 & 70 & 2.4090 & 2.3030 & 2.2920 \\
\hline 40 & 60 & 2.2930 & 2.1750 & 2.1540 \\
\hline 50 & 50 & 2.1760 & 2.2800 & 2.2410 \\
\hline 60 & 40 & 2.2870 & 2.3360 & 2.3370 \\
\hline 70 & 30 & 2.3880 & 2.6720 & 2.3423 \\
\hline 80 & 20 & 3.2320 & & 2.3510 \\
\hline 90 & 10 & 4.0120 & & \\
\hline
\end{tabular}

* peak was not observed

\section{a. Reproducibility study (\%RSD)}

The reproducibility study was conducted for this column using two amino acids, phenylalanine and phenylglycine, in 70:30 ACN:DI $\mathrm{H}_{2} \mathrm{O}$ mobile phase. Ten consecutive injections of the analytes resulted in retention times with close proximity, as shown in Table 14. The \% RSD values calculated and reported in Table 14, as well. The obtained \%RSD values range for this column is $0.3347-0.6111$ which is less than 1.0. The 
significance of this range verifies the high degree of precision in the 1,8-nonadiyne bonded stationary phase performance when obtaining the ANP retention data.

Table 14. Reproducibility for 10 injections of two amino acids using the 1,8-nonadiyne bonded stationary phase.

\begin{tabular}{|c|c|c|}
\hline & \multicolumn{2}{|c|}{ Retention time (min) } \\
\hline $\begin{array}{c}\text { Number of } \\
\text { injections }\end{array}$ & Phenylglycine & Phenylalanine \\
& in ACN:DI $\mathbf{H}_{2} \mathbf{O}$ (70:30) & in ACN:DI $\mathbf{H}_{\mathbf{2}} \mathbf{O}$ (70:30) \\
\hline 1 & 2.3430 & 3.9150 \\
\hline 2 & 2.3010 & 3.8850 \\
\hline 3 & 2.3525 & 3.9200 \\
\hline 4 & 2.1000 & 3.9150 \\
\hline 5 & 2.3430 & 3.9430 \\
\hline 6 & 2.3010 & 3.9000 \\
\hline 7 & 2.2985 & 3.9430 \\
\hline 8 & 2.3430 & 3.9100 \\
\hline 9 & 2.3550 & 3.8655 \\
\hline 10 & 2.4010 & 3.9150 \\
\hline \%RSD & $\mathbf{0 . 3 3 4 7}$ & $\mathbf{0 . 6 1 1 1}$ \\
\hline
\end{tabular}

\section{ii. Hydrophobic analytes}

Analytes listed in Tables 15 and 16 are non-polar compounds. The first group is from the steroid family. This group shows a small amount of polarity but can be 
neglected for the purpose of this study. However, the presence of the $-\mathrm{OH}$ group in the corticosterone structure could make this compound somewhat more polar than the rest of the analytes of this family.

Table 15. Retention times of the hydrophobic steroids using the 1,8-nonadiyne column.

\begin{tabular}{|c|c|c|c|c|c|c|}
\hline \multicolumn{2}{|c|}{$\begin{array}{c}\text { Conc. of the } \\
\text { mobile phase } \\
\text { (\%) }\end{array}$} & \multicolumn{5}{|c|}{$\begin{array}{l}\text { Retention time } \\
\text { (min) }\end{array}$} \\
\hline ACN & $\begin{array}{c}\text { DI } \\
\mathrm{H}_{2} \mathrm{O}\end{array}$ & Adrenosterone & $\begin{array}{c}\Delta^{4}- \\
\text { Androst } \\
\text { en-3,17- } \\
\text { dione }\end{array}$ & Esterone & Estradiol & Corticosterone \\
\hline 10 & 90 & 2.8300 & 16.1050 & 2.8380 & 2.3840 & $*$ \\
\hline 20 & 80 & 2.7500 & 13.3850 & 2.7539 & 2.3510 & $*$ \\
\hline 30 & 70 & 2.4590 & 10.6100 & 2.4500 & 2.3140 & 13.3230 \\
\hline 40 & 60 & 2.3790 & 6.0760 & 2.3740 & 2.3070 & 6.4830 \\
\hline 50 & 50 & 2.2320 & 4.2110 & 2.2400 & 2.2410 & 4.3340 \\
\hline 60 & 40 & 2.3270 & 5.8280 & 2.3330 & 2.2940 & 3.3960 \\
\hline 70 & 30 & 2.3410 & * & 2.6560 & 2.3337 & * \\
\hline 80 & 20 & 2.6190 & $*$ & $*$ & 2.3390 & * \\
\hline 90 & 10 & $*$ & $*$ & $*$ & 2.3530 & $*$ \\
\hline
\end{tabular}

* peak was not observed 
According to Table 15, neither of the analytes shows considerable ANP behavior as they elute at the early stages of analysis. However, the RP retention behavior for the column with respect to these compounds is more obvious. Figure 25 compares the retention time of three of the studied analytes. The retention maps for corticosterone and adrenosterone ( $\boldsymbol{\Delta}$ and $\bullet$, respectively) show more RP behavior using 1,8-nonadiyne bonded stationary phase.

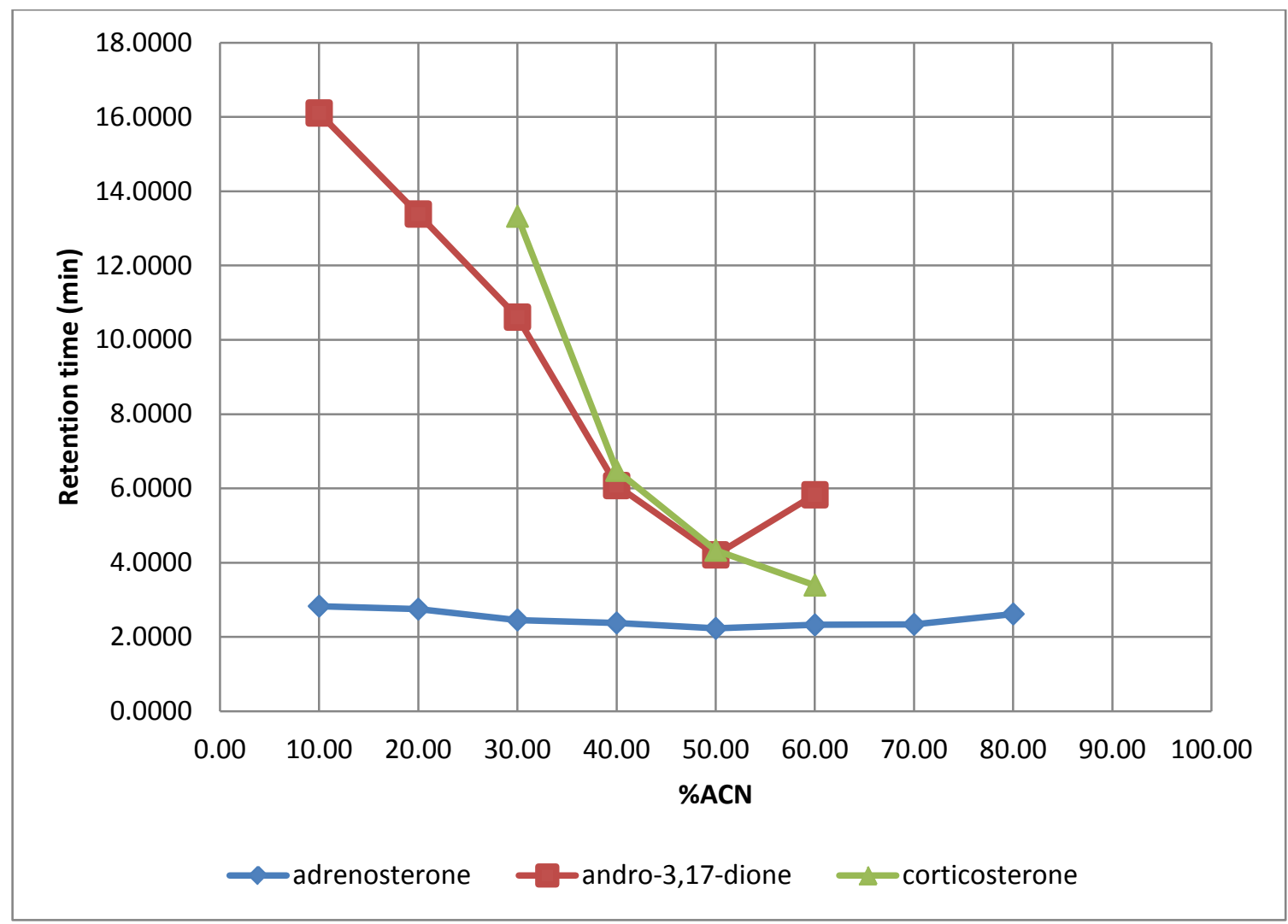

Figure 25. Retention map of the steroids, adrenosterone, $\Delta^{4}$-androsten-3,17-dione, and corticosterone using the 1,8-nonadiyne column

The next class of hydrophobic compounds for this study was polycyclic aromatic hydrocarbons. As expected, these compounds did not exhibit ANP behavior for this 
column. Table 15 reports the retention time of each analyte with the 1,8-nonadiyne column.

Table 16. Retention times of the hydrophobic polycyclic aromatic hydrocarbons using the 1,8-nonadiyne column.

\begin{tabular}{|c|c|c|c|c|c|}
\hline \multicolumn{2}{|c|}{$\begin{array}{c}\text { Roncentration of the } \\
\text { mobile phase (\%) }\end{array}$} & \multicolumn{4}{|c|}{ (min) } \\
\hline ACN & DI water & Naphthalene & Phenanthrene & Pyrene & Fluorene \\
\hline 10 & 90 & 2.3780 & $*$ & 2.3870 & 2.3770 \\
\hline 20 & 80 & 2.3590 & $*$ & 2.3490 & 2.3430 \\
\hline 30 & 70 & 2.3210 & $*$ & 2.3030 & 2.3280 \\
\hline 40 & 60 & 2.3000 & $*$ & 2.3140 & 2.2830 \\
\hline 50 & 50 & 2.1730 & 10.5930 & 2.1810 & 2.1850 \\
\hline 60 & 40 & 2.3010 & 5.7980 & 2.2920 & 2.2990 \\
\hline 70 & 30 & 2.3260 & 5.7880 & 2.3290 & 2.4100 \\
\hline 80 & 20 & 2.6780 & 4.1620 & 2.6830 & 2.6230 \\
\hline 90 & 10 & 2.7210 & 6.1020 & $*$ & $*$ \\
\hline
\end{tabular}

* peak was not observed

To better observe the effect of hydrophobicity vs. hydrophilicity of compounds with respect to RP/ ANP conditions, the retention map behaviors of three compounds with various degrees of polarity are plotted in Figure 26. As shown, the polar adenine ( $\bullet$ shows significant ANP behavior when the mobile phase hydrophobicity increases. In contrast, for a non-polar compound like $\Delta^{4}$-androsten-3,17-dione ( $\boldsymbol{\Delta}$ ), the RP behavior is very significant. Dopamine (-) shows a slight ANP behavior which is, to some extent, 
consistent with its mildly polar nature. The elution characteristics of the entire selection of polar molecules and non-polar molecules using the 1,8-nonadiyne as the bonded stationary phase for this study are compared in Figure 27.

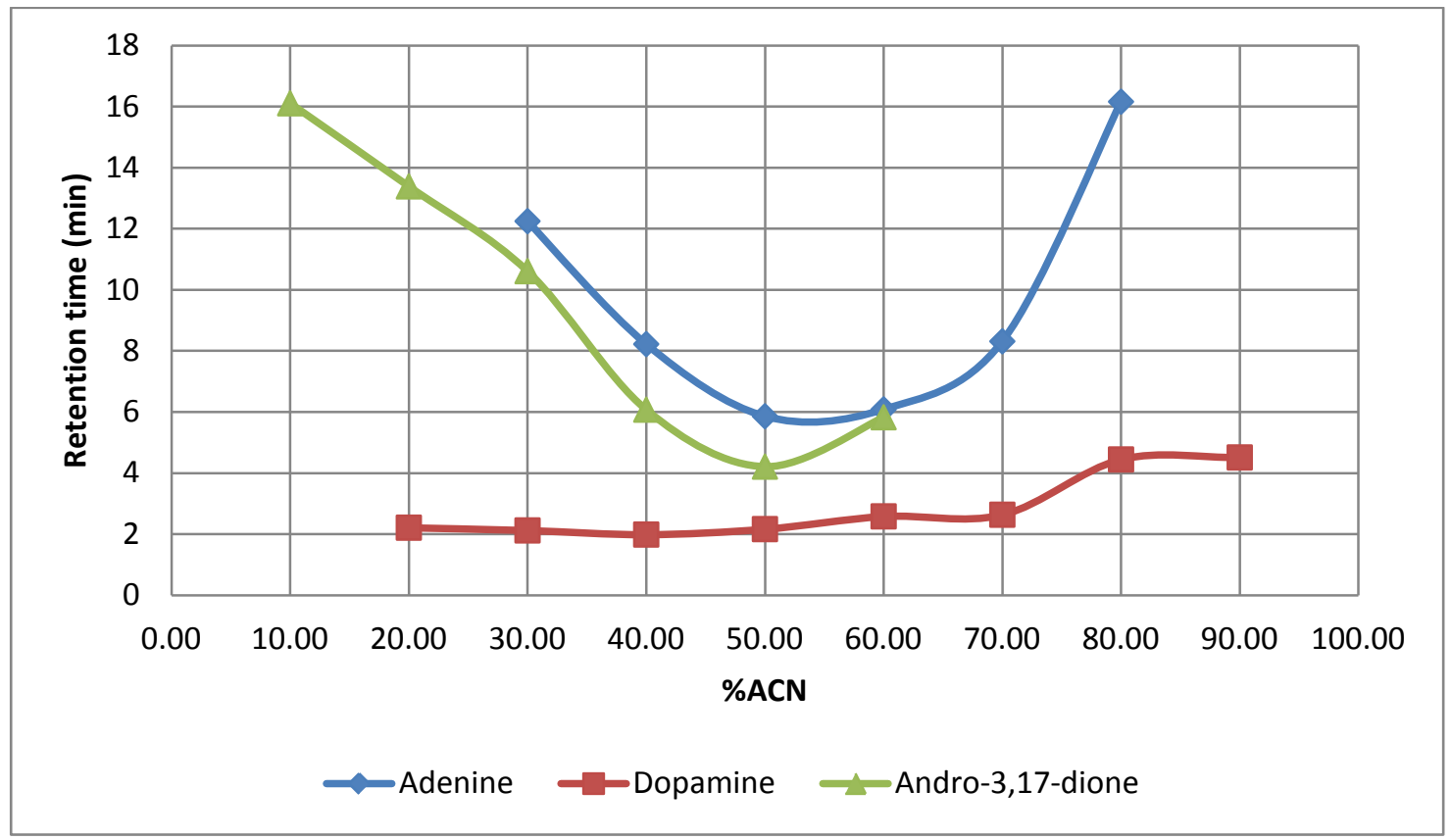

Figure 26. Retention map of adenine, dopamine, and $\Delta^{4}$-androsten-3,17-dione, using the 1,8-nonadiyne column 


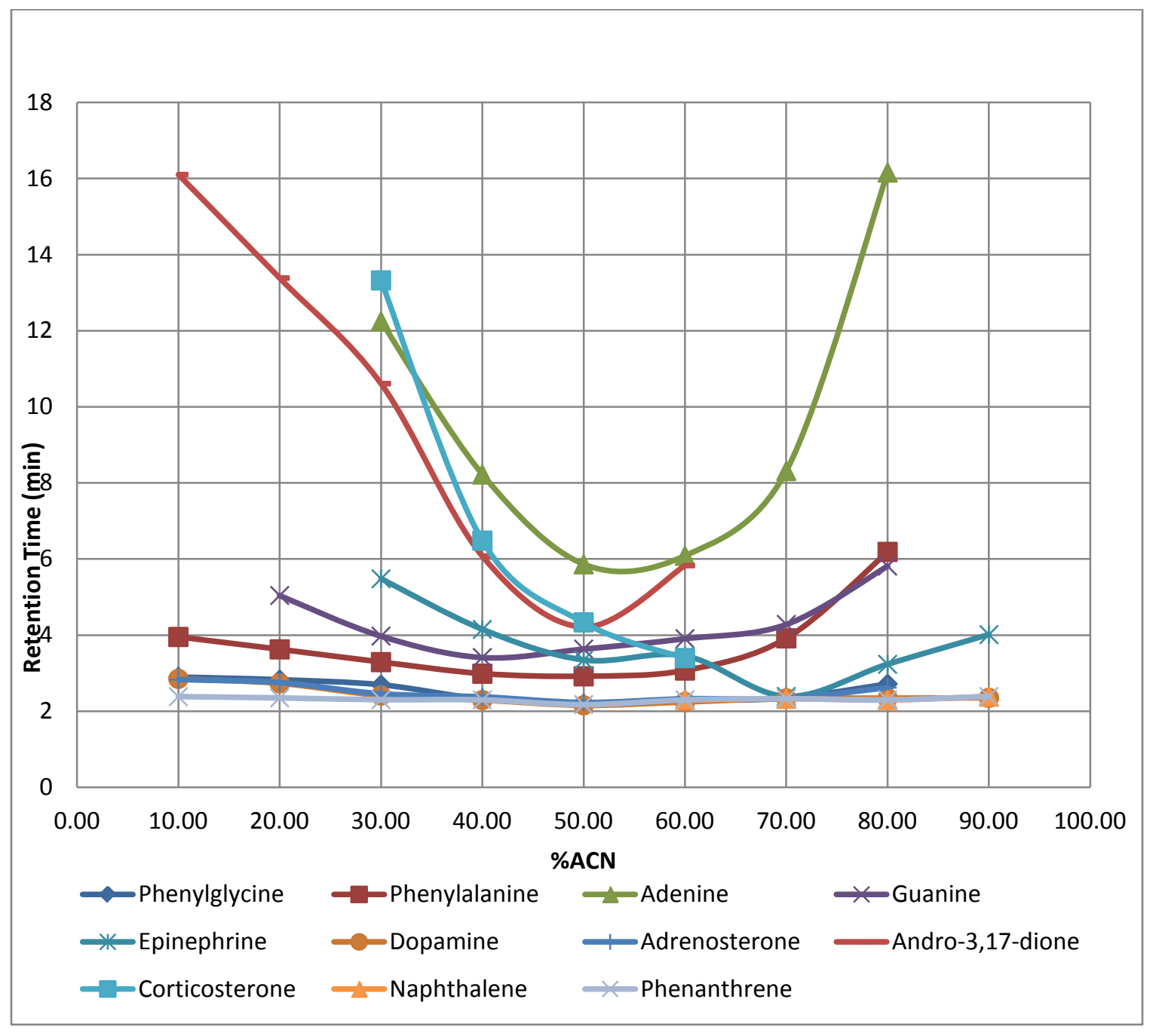

Figure 27. Retention map of the polar and non-polar analytes using the 1,8-nonadiyne column

\section{iii. Addition of formic acid (buffer)}

To further study the retention behavior of the class of analytes with the 1,8nonadiyne stationary phase, formic acid was used as a buffer. HPLC analysis confirmed that, when $0.1 \%(\mathrm{v} / \mathrm{v})$ formic acid was added to the binary solvent mobile phase, it enhanced the ANP behavior of the compounds toward the stationary phase. Figure 28 shows the effect of the formic acid (FA) additive on two polar analytes. The effect is 
better observed when compared with the similar conditions exclusive of buffer, as shown in Figure 29. The studies, both with and without formic acid buffer, for the 1,8nonadiyne in the presence of a wide range of hydrophilic and hydrophobic analytes show that the column exhibits both RP and ANP behavior.

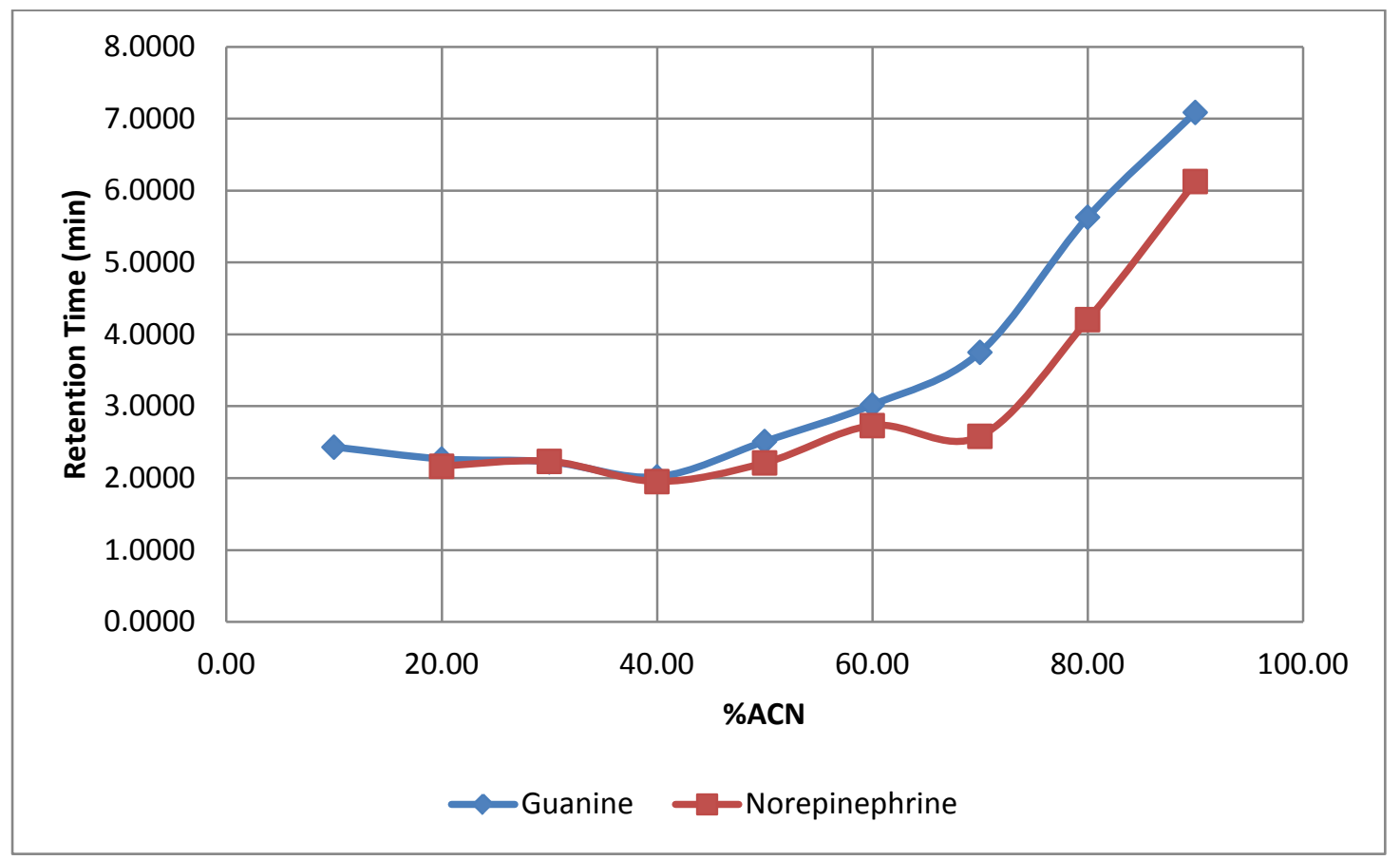

Figure 28. Retention map of two polar analytes using the 1,8-nonadiyne column and $0.1 \%$ formic acid in the mobile phase 


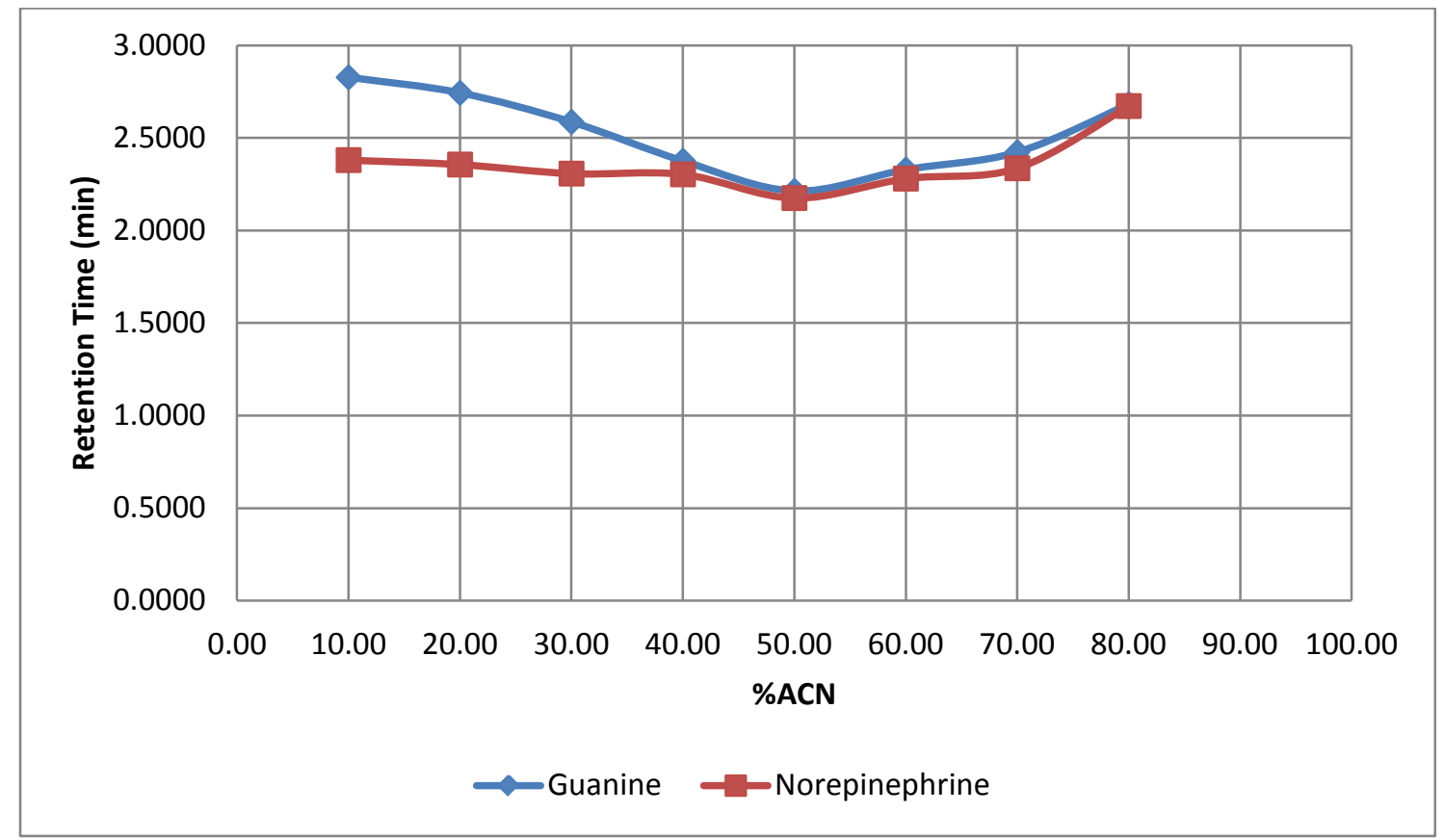

Figure 29. Retention map of two polar analytes using the 1,8-nonadiyne column without buffer

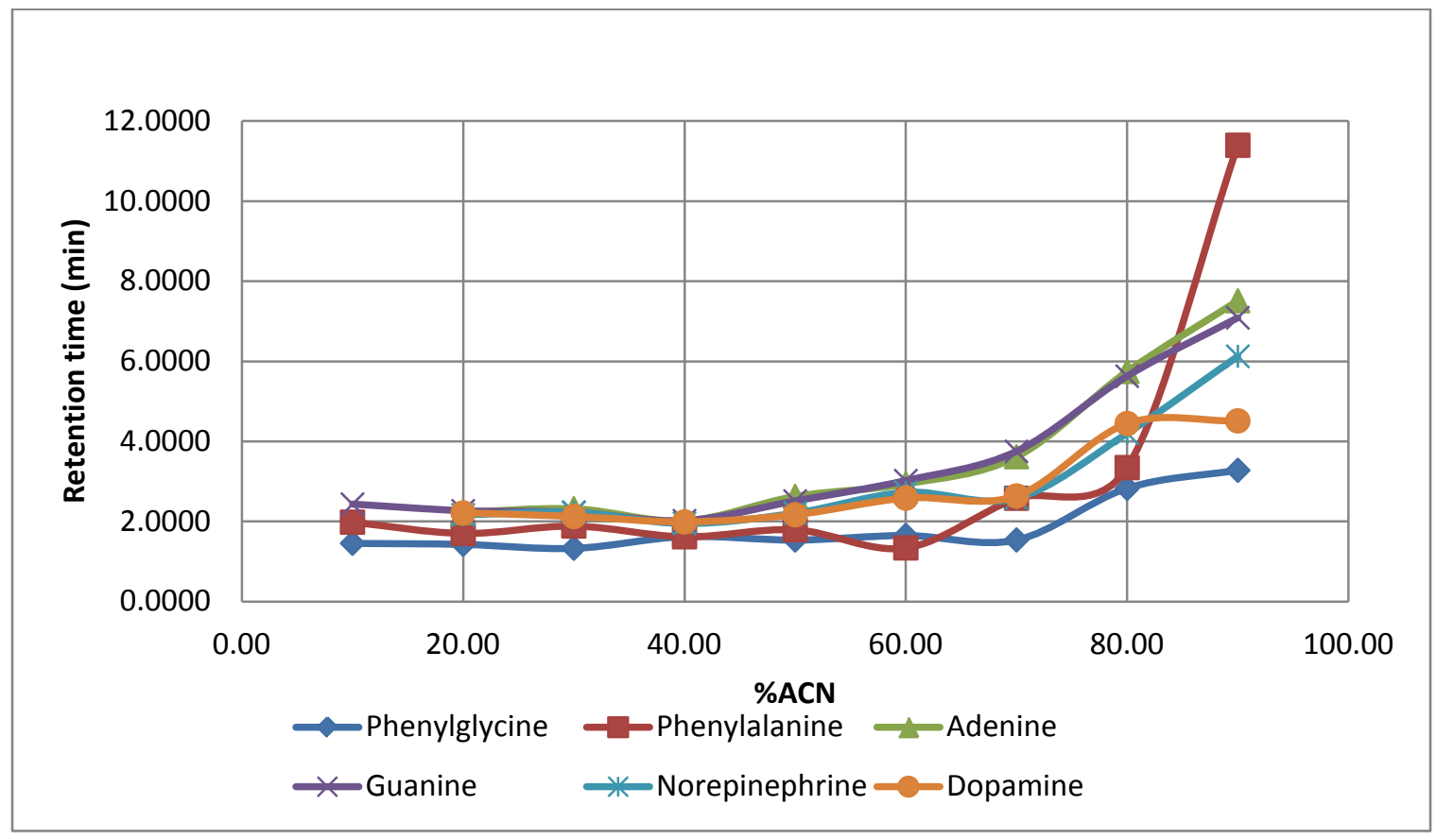

Figure 30. Retention map of the polar analytes using the 1,8-nonadiyne column and $0.1 \%$ FA in the mobile phase 
Table 17. Retention times of the hydrophilic analytes using the 1,8-nonadiyne column with $0.1 \%$ formic acid buffer in the mobile phase.

\begin{tabular}{|c|c|c|c|c|c|c|c|c|}
\hline \multicolumn{2}{|c|}{$\begin{array}{c}\text { Conc. of the mobile } \\
\text { phase (\%) }\end{array}$} & \multicolumn{7}{|c|}{ Retention time (min) } \\
\hline $\begin{array}{l}\text { ACN \& } \\
0.1 \% \text { FA }\end{array}$ & $\begin{array}{c}\mathrm{H}_{2} \mathrm{O} \& \\
0.1 \% \text { FA }\end{array}$ & PhG & PhA & Adn & Gun & Epi & Nepi & Dopa \\
\hline 10 & 90 & 1.4550 & 1.9820 & $*$ & 2.4300 & $*$ & $*$ & $*$ \\
\hline 20 & 80 & 1.4290 & 1.7030 & 2.2240 & 2.2650 & 3.5240 & 2.1640 & 2.2130 \\
\hline 30 & 70 & 1.3310 & 1.8860 & 2.3190 & 2.2240 & 2.4010 & 2.2320 & 2.1230 \\
\hline 40 & 60 & 1.6240 & 1.6180 & 2.0170 & 2.0170 & 2.8960 & 1.9510 & 1.9880 \\
\hline 50 & 50 & 1.5370 & 1.7940 & 2.6260 & 2.5090 & 8.0010 & 2.2110 & 2.1660 \\
\hline 60 & 40 & 1.6510 & 1.3400 & 2.9380 & 3.0180 & 3.7840 & 2.7300 & 2.5830 \\
\hline 70 & 30 & 1.5380 & 2.5800 & 3.6170 & 3.7490 & 3.5110 & 2.5810 & 2.6370 \\
\hline 80 & 20 & 2.8260 & 3.3450 & 5.7450 & 5.6280 & 2.9960 & 4.2040 & 4.4460 \\
\hline 90 & 10 & 3.2770 & 11.3940 & 7.5110 & 7.0860 & 3.0170 & 6.1250 & 4.5120 \\
\hline
\end{tabular}

* peak was not observed

\begin{tabular}{|l|l|l|l|}
\hline PhG $=$ & PhA $=$ & Adn $=$ & Gun $=$ \\
Phenylglycine & Phenylalanine & Adenine & Guanine \\
\hline Epi $=$ & Nepi $=$ & Dopa $=$ & FA = Formic \\
Epinephrine & Norepinephrine & Dopamine & acid \\
\hline
\end{tabular}




\section{5-Cyano-1-pentyne}

\section{i. Hydrophilic analytes}

The RP/ ANP behavior of 5-cyano-1-pentyne stationary phase was studied using similar analytes and mobile phases. The results are tabulated, plotted, and presented in the following pages. There was, however, one unusual behavior exclusive to this column. The column, at relatively low concentrations of ACN in the mobile phase, (i.e., $\geq 30 \%$, had no observable peaks). In other words, the chromatograms appeared in the form of random noise. This unusual behavior can be explained by possible irreversible adsorption of the analytes on the stationary phase. However, when $0.1 \%(\mathrm{v} / \mathrm{v})$ formic acid buffer was added, this abnormality disappeared.

Tables 18, 19, and 20 show retention times for the three groups of hydrophilic analytes when testing the 5-cyano-1-pentyne bonded stationary phase with mobile phases ranging from polar to less polar. The collected data, as well as the retention maps, for nucleobases (adenine and guanine) and neurotransmitters (epinephrine, norepinephrine, dopamine) shown in Figures 31 and 32 confirm that 5-cyano-1-pentyne exhibits ANP behavior. 
Table 18. Retention times of the hydrophilic amino acids using the 5-cyano-1-pentyne column.

\begin{tabular}{|c|c|c|c|}
\hline \multicolumn{2}{|c|}{ Concentration of the } & \multicolumn{2}{c|}{ Retention time } \\
mobile phase (\%) & Phenylglycine & Phenylalanine \\
\hline Acetonitrile & DI water & $*$ & $*$ \\
\hline 10 & 90 & $*$ & $*$ \\
\hline 20 & 80 & 3.145 & 2.0850 \\
\hline 30 & 70 & 2.140 & 2.8670 \\
\hline 40 & 60 & 2.5420 & 3.3150 \\
\hline 50 & 50 & 3.0370 & 4.7720 \\
\hline 60 & 40 & 4.2380 & $*$ \\
\hline 70 & 30 & $*$ & \\
\hline 80 & 20 & & \\
\hline 90 & 10 & & \\
\hline
\end{tabular}


Table 19. Retention times of the hydrophilic nucleobases using the 5-cyano-1-pentyne column.

\begin{tabular}{|c|c|c|c|}
\hline \multicolumn{2}{|c|}{ Concentration of the mobile } & \multicolumn{2}{c|}{ (min) } \\
\hline phase (\%) & Adenine & Guanine \\
\hline 10 & 90 & $*$ & $*$ \\
\hline 20 & 80 & $*$ & $*$ \\
\hline 30 & 70 & 3.0260 & 2.1220 \\
\hline 40 & 60 & 2.3670 & 2.0000 \\
\hline 50 & 50 & 4.9080 & 2.8530 \\
\hline 60 & 40 & 5.1990 & 3.3710 \\
\hline 70 & 30 & 6.0250 & 4.7140 \\
\hline 80 & 20 & 7.9250 & \\
\hline 90 & 10 & & \\
\hline
\end{tabular}

* peak was not observed 


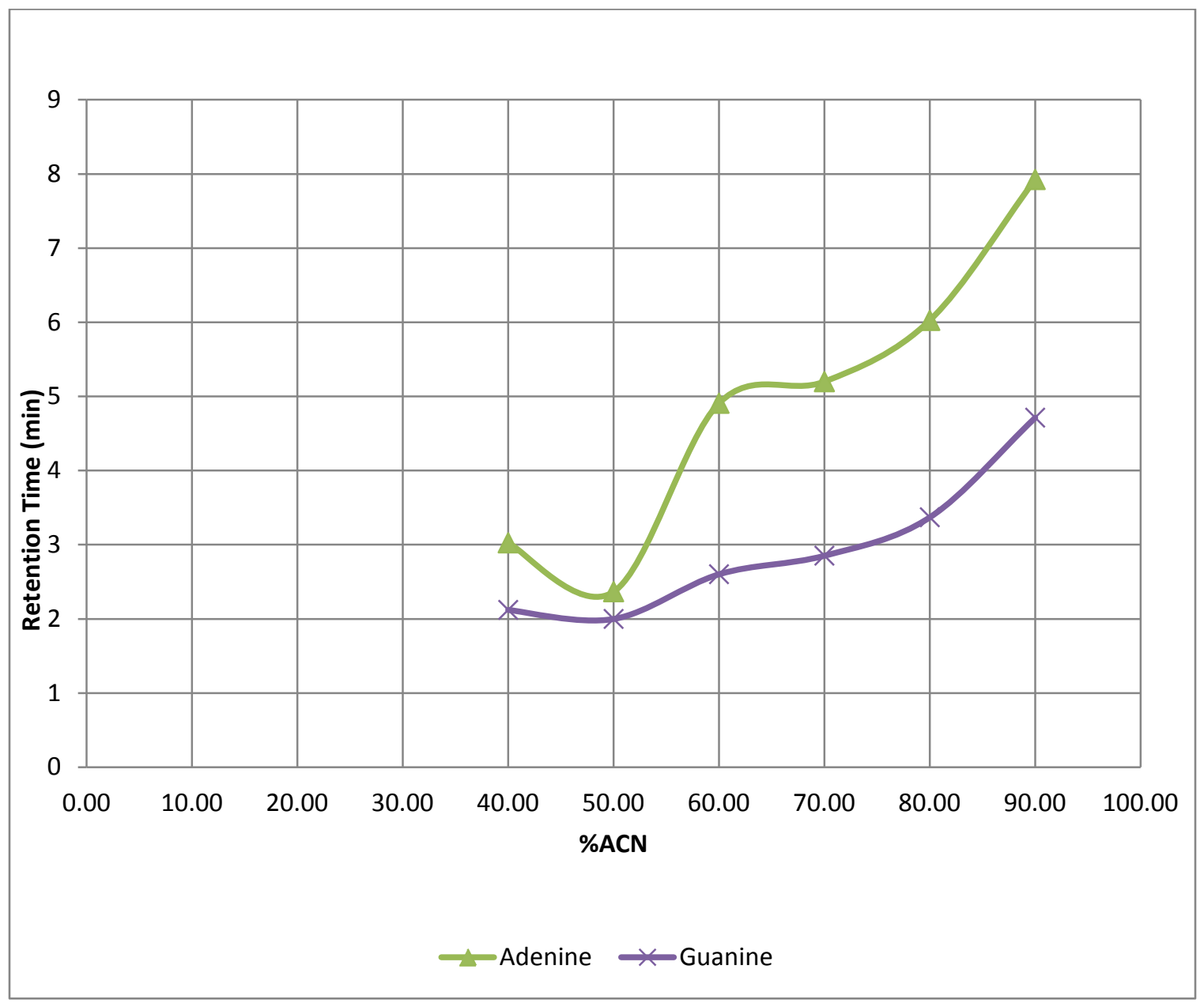

Figure 31. Retention map of the polar nucleobases using the 5-cyano-1-pentyne column 
Table 20. Retention times of the hydrophilic neurotransmitters using the 5-cyano-1pentyne column.

\begin{tabular}{|c|c|c|c|c|}
\hline \multicolumn{2}{|c|}{ Concentration of the } & \multicolumn{3}{|c|}{ Retention time } \\
mobile phase (\%) & & Norepinephrine & Dopamine \\
\hline Acetonitrile & DI water & Epinephrine & $*$ & $*$ \\
\hline 10 & 90 & $*$ & $*$ & $*$ \\
\hline 20 & 80 & $*$ & $*$ & 1.8980 \\
\hline 30 & 70 & $*$ & 1.6680 & 1.8340 \\
\hline 40 & 60 & 1.8850 & 1.8790 & 1.8910 \\
\hline 50 & 50 & 1.6660 & 1.8860 & 1.8970 \\
\hline 60 & 40 & 1.8790 & 1.9180 & 1.9380 \\
\hline 70 & 30 & 2.4850 & 1.9350 & 2.2340 \\
\hline 80 & 20 & 2.8600 & & \\
\hline 90 & 10 & 3.6810 & & \\
\hline
\end{tabular}

\footnotetext{
* peak was not observed
} 


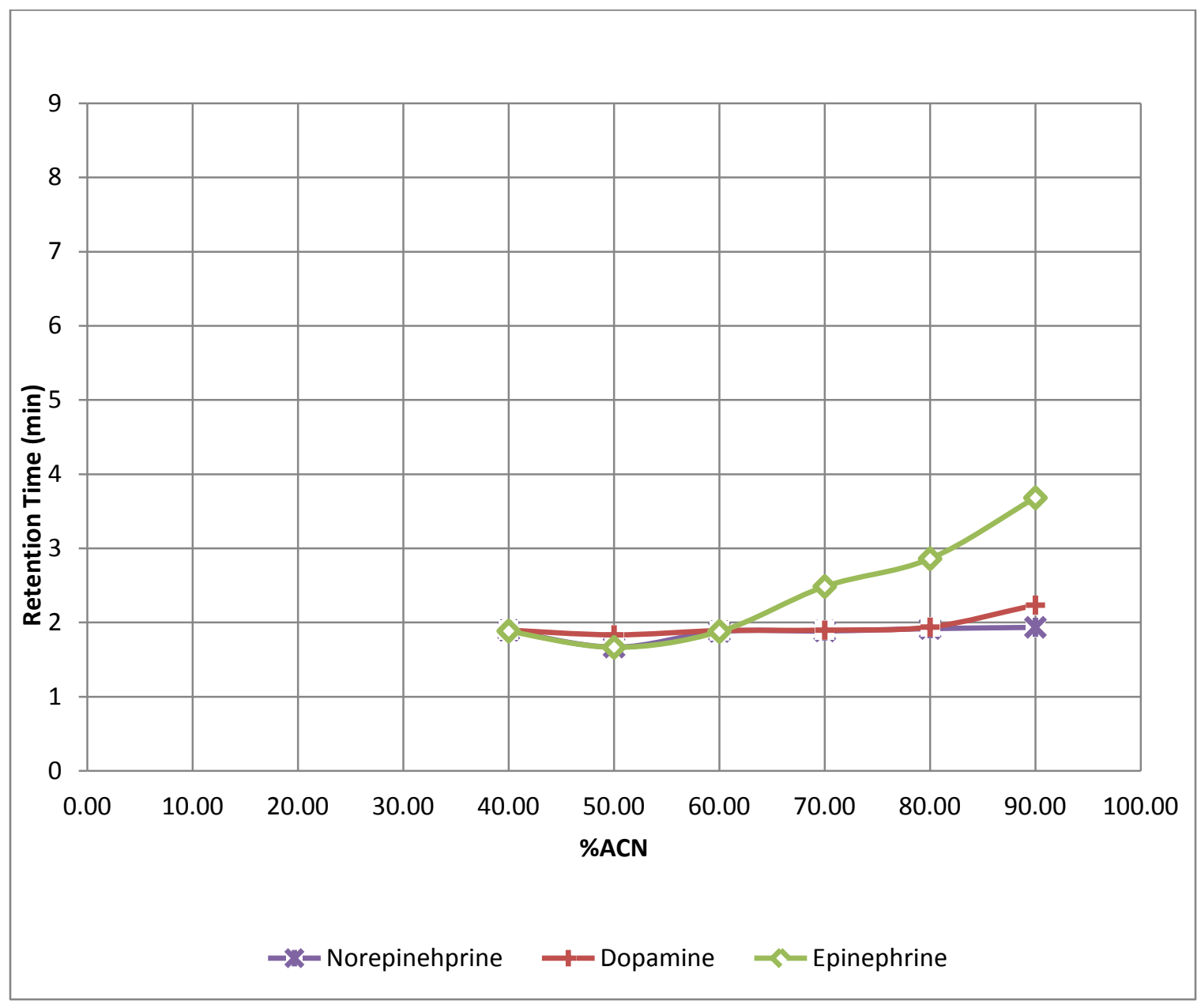

Figure 32. Retention map of the polar neurotransmitters using the 5-cyano-1-pentyne column

\section{ii. Hydrophobic analytes}

The retention times for the steroid molecules are short. However, from the retention maps for adrenesterone and estradiol shown in Figure 33 (+ and -), one can observe slight ANP behavior for the molecules when testing the 5-cyano-1-pentyne column. The ANP behavior is more substantial for phenanthrene from the polycyclic aromatic hydrocarbons, as shown in Figure $34(><)$. Figure 35 compares the retention behavior of some of the non-polar and polar analytes using the 5-cyano-1-pentyne 
stationary phase. The retention times of the hydrophobic analytes, steroids, and polycyclic aromatic hydrocabons, using various concentrations of the mobile phase for the 5-cyano-1-pentyne bonded stationary phase, are provided in Tables 21 and 22.

Table 21. Retention times of the hydrophobic steroids using the 5-cyano-1-pentyne column.

\begin{tabular}{|c|c|c|c|c|c|c|}
\hline \multicolumn{2}{|c|}{$\begin{array}{c}\text { mobile phase } \\
\text { conc. }\end{array}$} & \multicolumn{5}{|c|}{ Retention time (min) } \\
\hline $\mathbf{A C N}$ & $\begin{array}{c}\text { DI } \\
\mathrm{H}_{2} \mathrm{O}\end{array}$ & $\begin{array}{l}\text { Adreno } \\
\text { sterone }\end{array}$ & $\begin{array}{l}\Delta^{4} \text {-Andro } \\
\text { 3,17-dione }\end{array}$ & Esterone & Estradiol & Corticosterone \\
\hline 10 & 90 & $*$ & $*$ & $*$ & $*$ & $*$ \\
\hline 20 & 80 & $*$ & $*$ & $*$ & $*$ & $*$ \\
\hline 30 & 70 & $*$ & $*$ & $*$ & $*$ & $*$ \\
\hline 40 & 60 & 1.9140 & 1.8960 & 1.8980 & 1.9020 & 1.8980 \\
\hline 50 & 50 & 1.6970 & 1.6940 & 1.6800 & 1.6850 & 1.6870 \\
\hline 60 & 40 & 1.9070 & 1.8830 & 1.8490 & 1.5920 & 1.8810 \\
\hline 70 & 30 & 1.8990 & 1.8950 & 1.8890 & 1.7960 & 1.8870 \\
\hline 80 & 20 & 1.9050 & 1.9130 & 1.9130 & 1.8940 & 1.9170 \\
\hline 90 & 10 & 1.9330 & 1.9200 & 1.9410 & 1.9320 & 1.9340 \\
\hline
\end{tabular}

* peak was not observed 


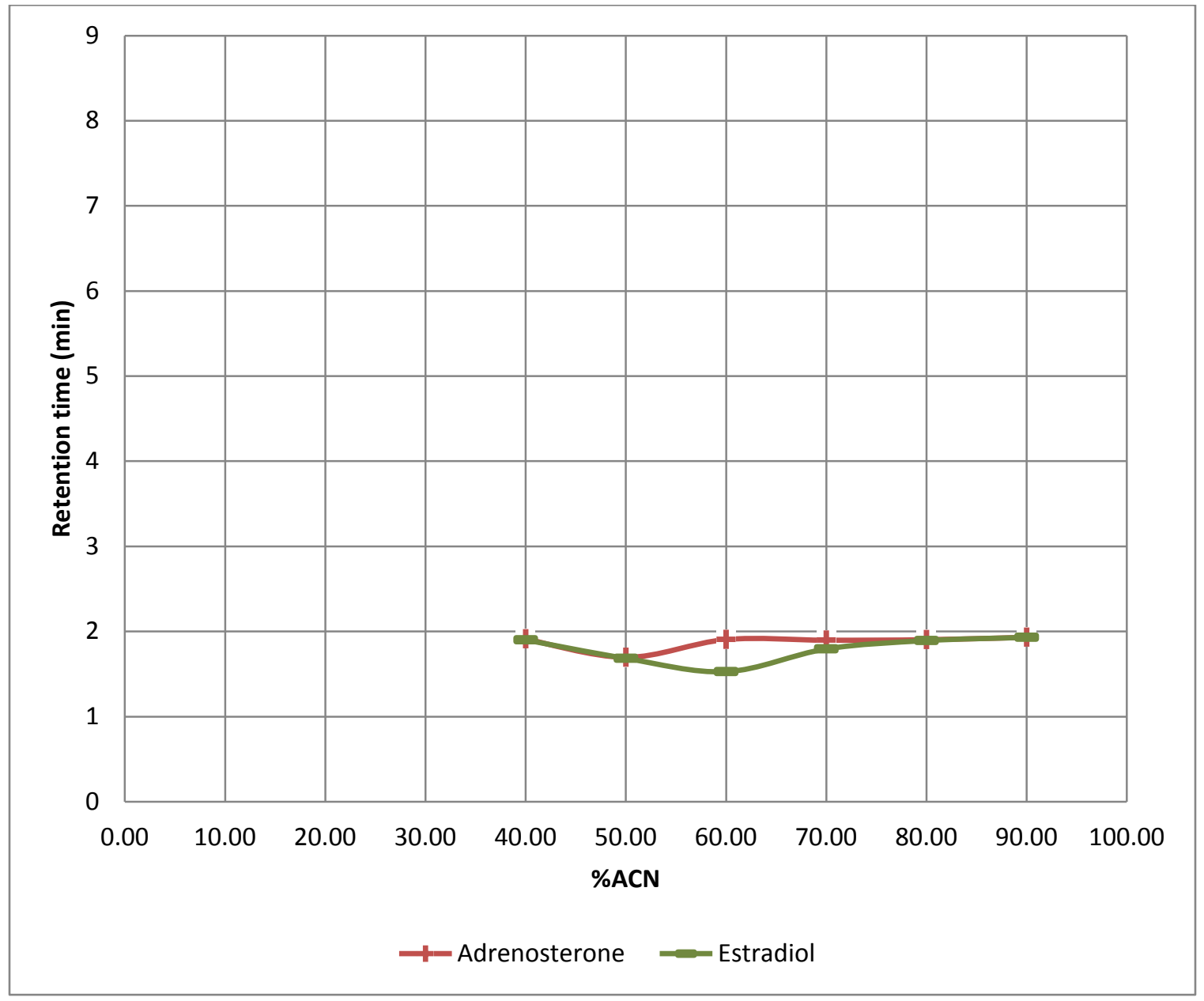

Figure 33. Retention map of the non-polar steroids using the 5-cyano-1-pentyne column 
Table 22. Retention times of the hydrophobic polycyclic aromatic hydrocarbons using the 5-cyano-1-pentyne column.

\begin{tabular}{|c|c|c|c|c|c|}
\hline \multicolumn{2}{|c|}{ Concentration of the } & \multicolumn{5}{|c|}{ Retention time } \\
mobile phase (\%) & & \multicolumn{5}{c|}{} \\
\hline ACN & DI water & Naphthalene & Phenantherene & Pyrene & Flourene \\
\hline 10 & 90 & $*$ & $*$ & $*$ & $*$ \\
\hline 20 & 80 & $*$ & $*$ & $*$ & $*$ \\
\hline 30 & 70 & $*$ & $*$ & $*$ & $*$ \\
\hline 40 & 60 & 1.8990 & 2.1930 & 2.3310 & 1.8990 \\
\hline 50 & 50 & 1.7110 & 1.97902 & 1.9770 & 1.6880 \\
\hline 60 & 40 & 1.8940 & 2.7010 & 1.8230 & 1.8760 \\
\hline 70 & 30 & 1.8740 & 3.2900 & 1.8850 & 1.8890 \\
\hline 80 & 20 & 1.9050 & 4.6600 & 1.9180 & 1.9170 \\
\hline 90 & 10 & 1.9450 & $*$ & 1.9410 & 1.9370 \\
\hline$*$
\end{tabular}

* peak was not observed 


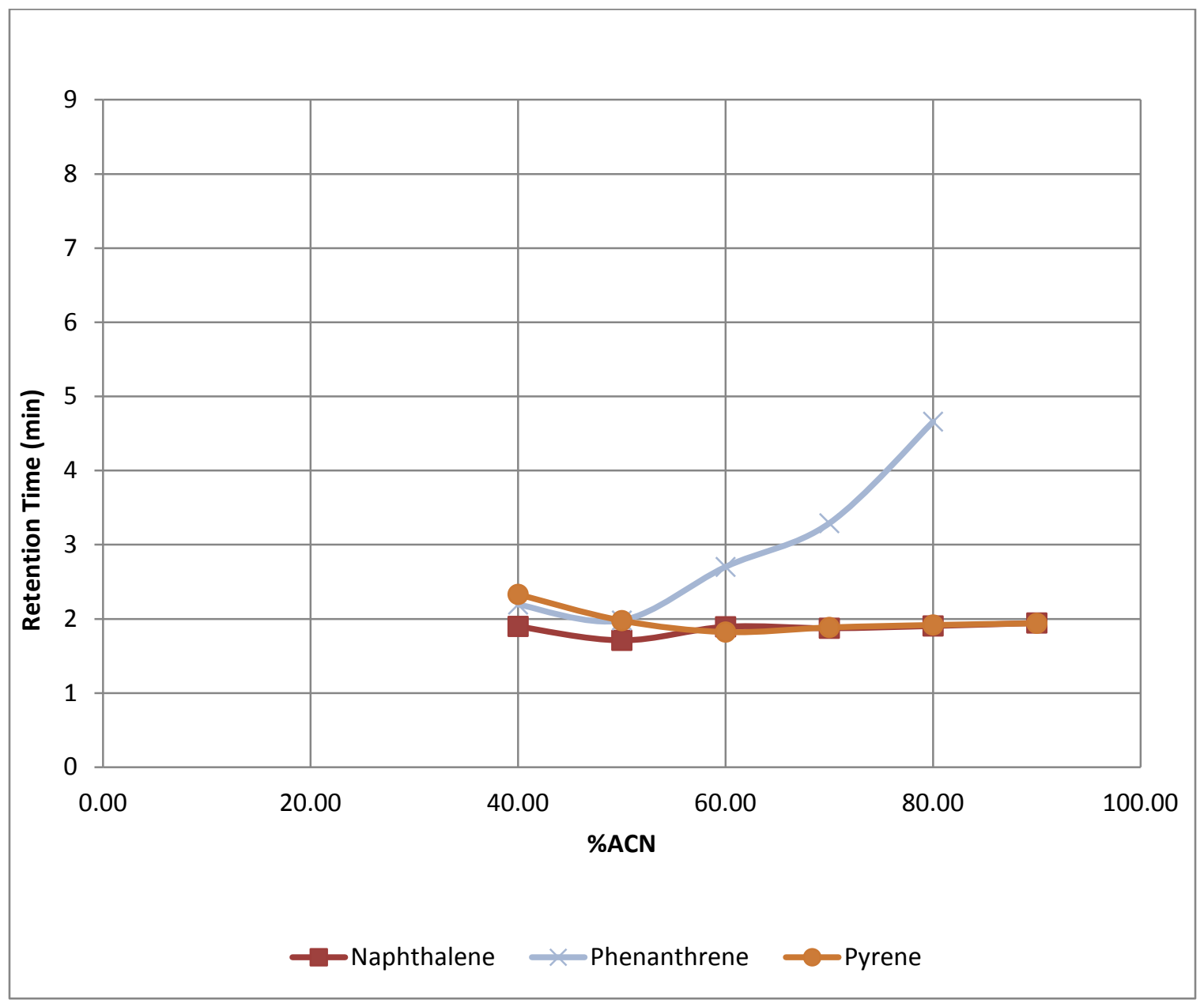

Figure 34. Retention map of the non-polar analytes using the 5-cyano-1-pentyne column 


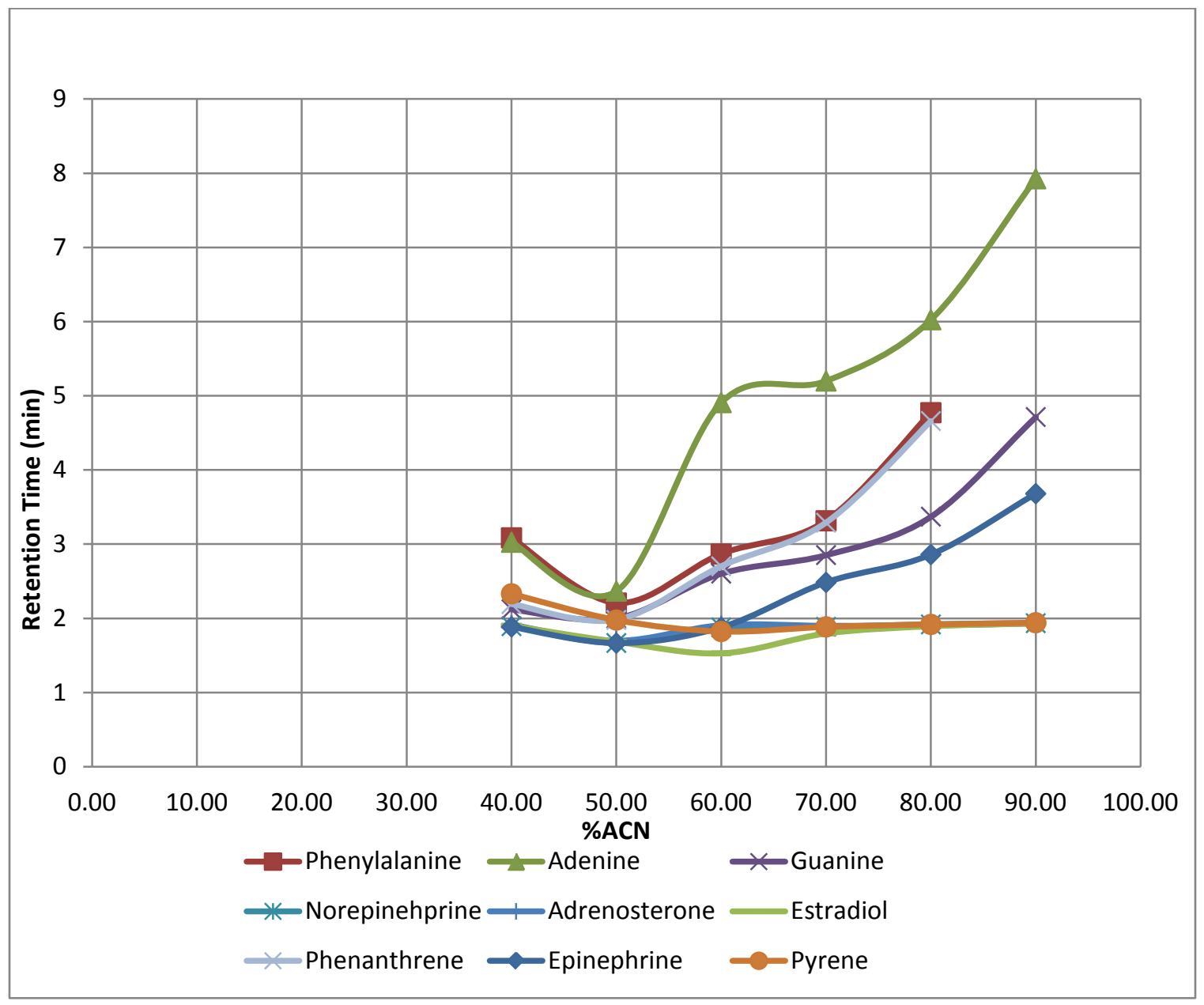

Figure 35. Retention map of the polar and non-polar analytes using the 5-cyano-1pentyne column

Studying the retention maps of the various analytes, one can determine that the column shows more ANP behavior. One plausible explanation for the 5-cyano-1-pentyne column having ANP behavior only could be the presence of the $\mathrm{C} \equiv \mathrm{N}$ bond, which makes the molecule moderately polar. 


\section{iii. Addition of formic acid (buffer)}

To observe the effect of buffer on the retention behavior of the compounds on the 5-cyano-1-pentyne bonded stationary phase, two molecules with different properties are chosen. A comparison of a molecule with typical reversed-phase behavior, pyrene, to one with typical ANP behavior, epinephrine, in the presence of 0/1\% (v/v) formic acid in the mobile phase is tabulated and plotted in Table 23 and Figure 36. An interesting feature of this plot is that, in the presence of buffer, both molecules exhibit RP and ANP behavior. This phenomenon could be due to the interaction of the buffer with the $\mathrm{C} \equiv \mathrm{N}$ functional group on 5-cyano-1-pentyne, the absence of irreversible adsorption of molecules on the surface, or a combination of these possibilities. 
Table 23. Retention times of epinephrine and pyrene using the 5-cyano-1-pentyne column with $0.1 \%$ formic acid buffer in the mobile phase.

\begin{tabular}{|c|c|c|c|}
\hline \multicolumn{2}{|c|}{ Concentration of the } & \multicolumn{2}{c|}{ (min) } \\
mobile phase (\%) & Epinephrine & Pyrene \\
0.1\% FA & FA & (hydrophilic) & (hydrophobic) \\
\hline 10 & 90 & 4.3170 & 3.1300 \\
\hline 20 & 80 & 2.7040 & 2.6370 \\
\hline 30 & 70 & 2.3090 & 3.41110 \\
\hline 40 & 60 & 2.1130 & 2.3600 \\
\hline 50 & 50 & 2.3870 & 2.4090 \\
\hline 60 & 40 & 2.6970 & 2.6190 \\
\hline 70 & 30 & 3.2160 & 3.1260 \\
\hline 80 & 20 & 3.5790 & \\
\hline 90 & 10 & 6.1970 & \\
\hline & & & \\
\hline
\end{tabular}




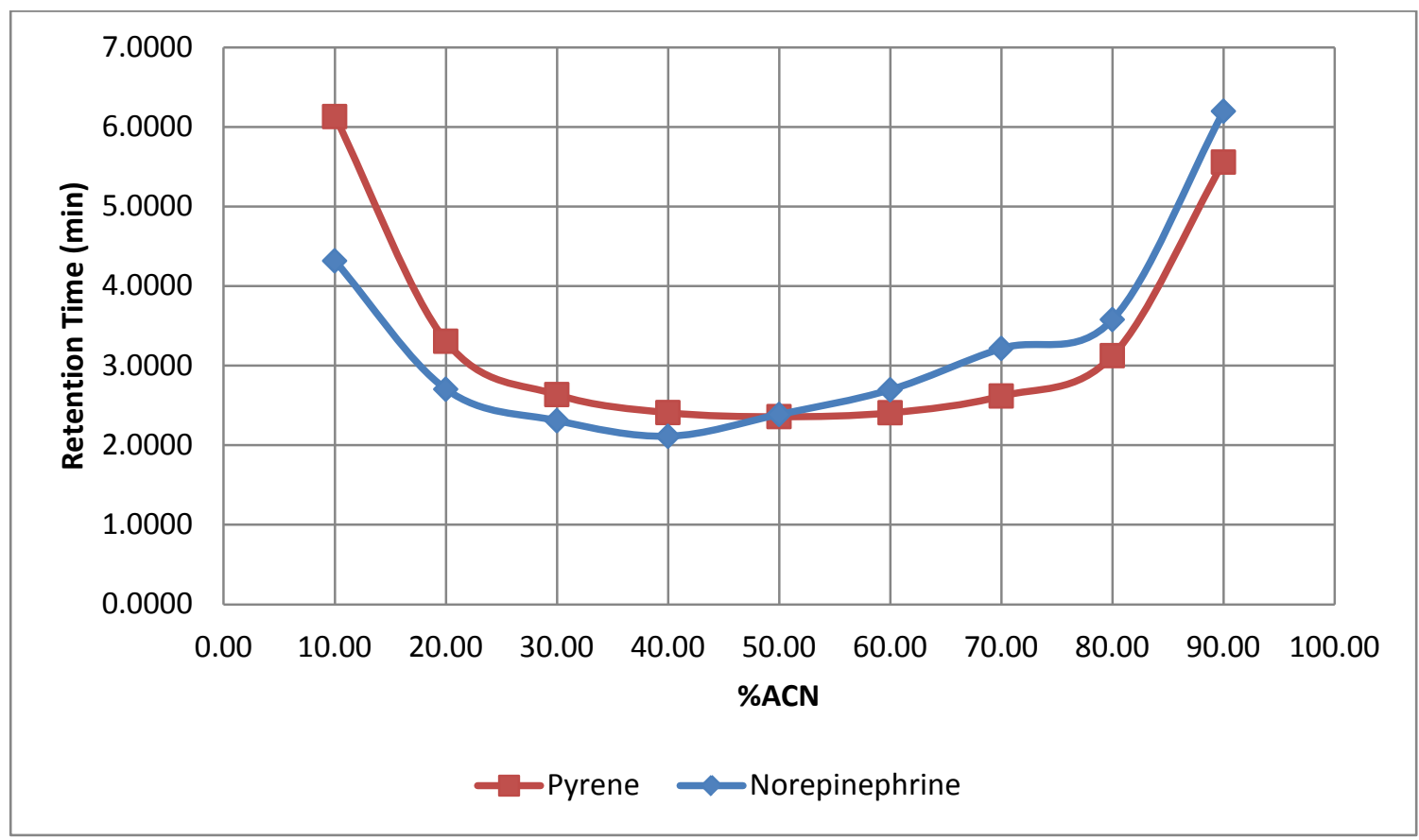

Figure 36. Retention map of a polar and non-polar analytes using the 5-cyano-1-pentyne column and $0.1 \%$ formic acid in the mobile phase

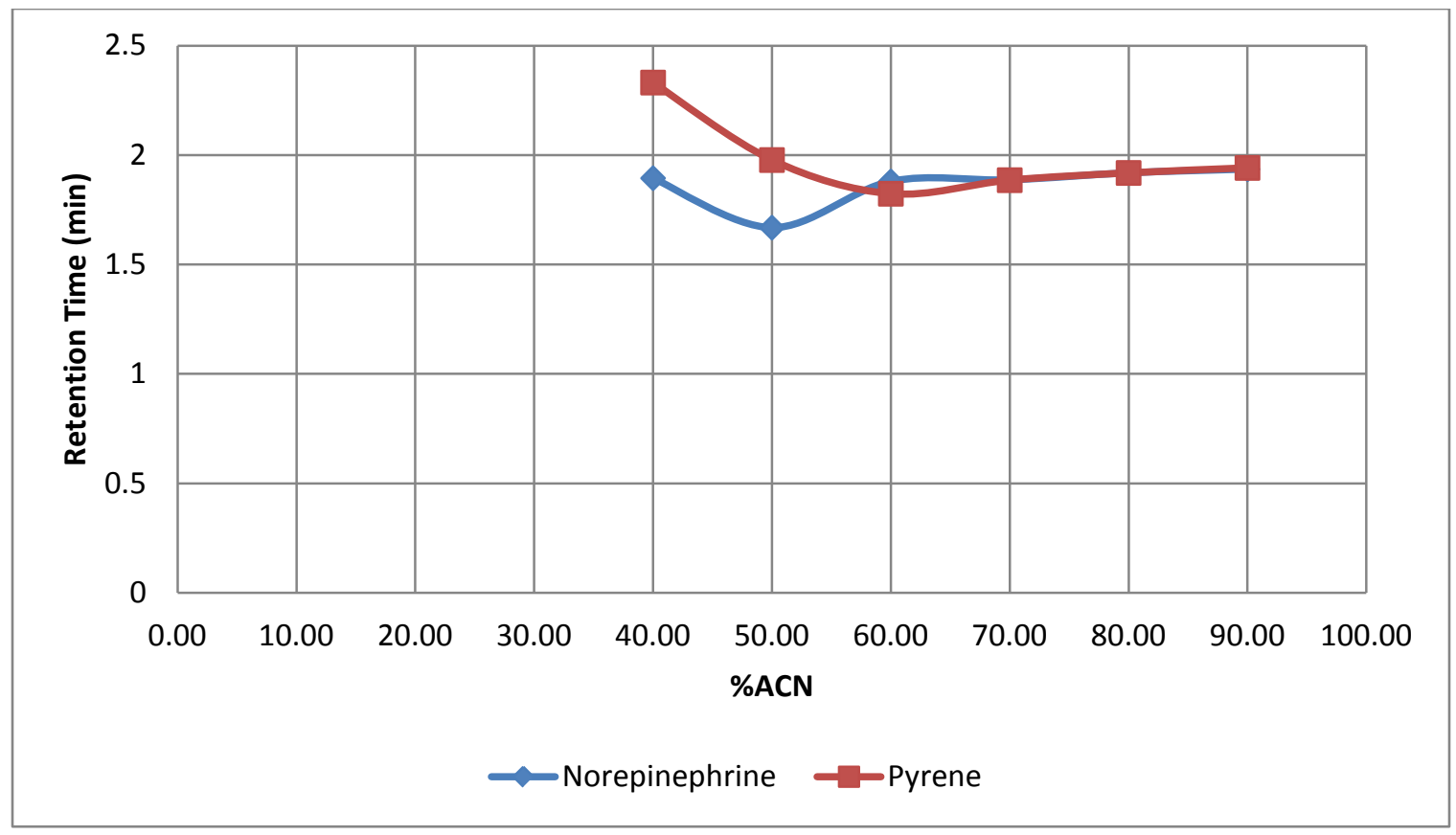

Figure 37. Retention map of a polar and non-polar analytes using the 5-cyano-1-pentyne column without buffer 


\section{6-Chloro-1-hexyne}

\section{i. Hydrophilic analytes}

The next bonded stationary phase to be evaluated was 6-chloro-1-hexyne. The results from running the column with the two amino acids, phenylglycine and phenylalanine, under isocratic condition of the mobile phase of acetornitrile : DI water are in Table 24.

Table 24. Retention times of the hydrophilic amino acids using the 6-chloro-1-hexyne column.

\begin{tabular}{|c|c|c|c|}
\hline \multicolumn{2}{|c|}{$\begin{array}{c}\text { Retention time } \\
\text { (min) } \\
\text { phase (\%) }\end{array}$} & Phenylalanine \\
\hline Acetonitrile & DI water & Phenylglycine & 3.2280 \\
\hline 10 & 90 & 2.1690 & 2.8390 \\
\hline 20 & 80 & 2.0470 & 2.5980 \\
\hline 30 & 70 & 2.0010 & 2.2940 \\
\hline 40 & 60 & 1.8900 & 2.6970 \\
\hline 50 & 50 & 1.7310 & 3.3170 \\
\hline 60 & 40 & 2.4390 & 4.2220 \\
\hline 70 & 30 & 3.1150 & 6.1650 \\
\hline 80 & 20 & 4.3760 & $*$ \\
\hline 90 & 10 & $*$ & \\
\hline
\end{tabular}

* peak was not observed 
As shown in Figure 38, the retention maps for both phenylglycine and phenylalanine ( $\downarrow$ and $\mathbf{m}$, respectively) are similar in terms of ANP/ RP properties of the column. In other words, the 6-chloro-1-hexyne bonded stationary phase exhibits both ANP and RP retention behavior at the same time. In addition, the column had great reproducibility. The testing of the column with 5 consecutive runs showed the same retention time $\pm 0.05 \mathrm{~min}$. Similar ANP/ RP results were obtained with adenine and guanine ( $\bullet$ and $\boldsymbol{\Delta}$, respectively) retention maps as shown in Figure 39.

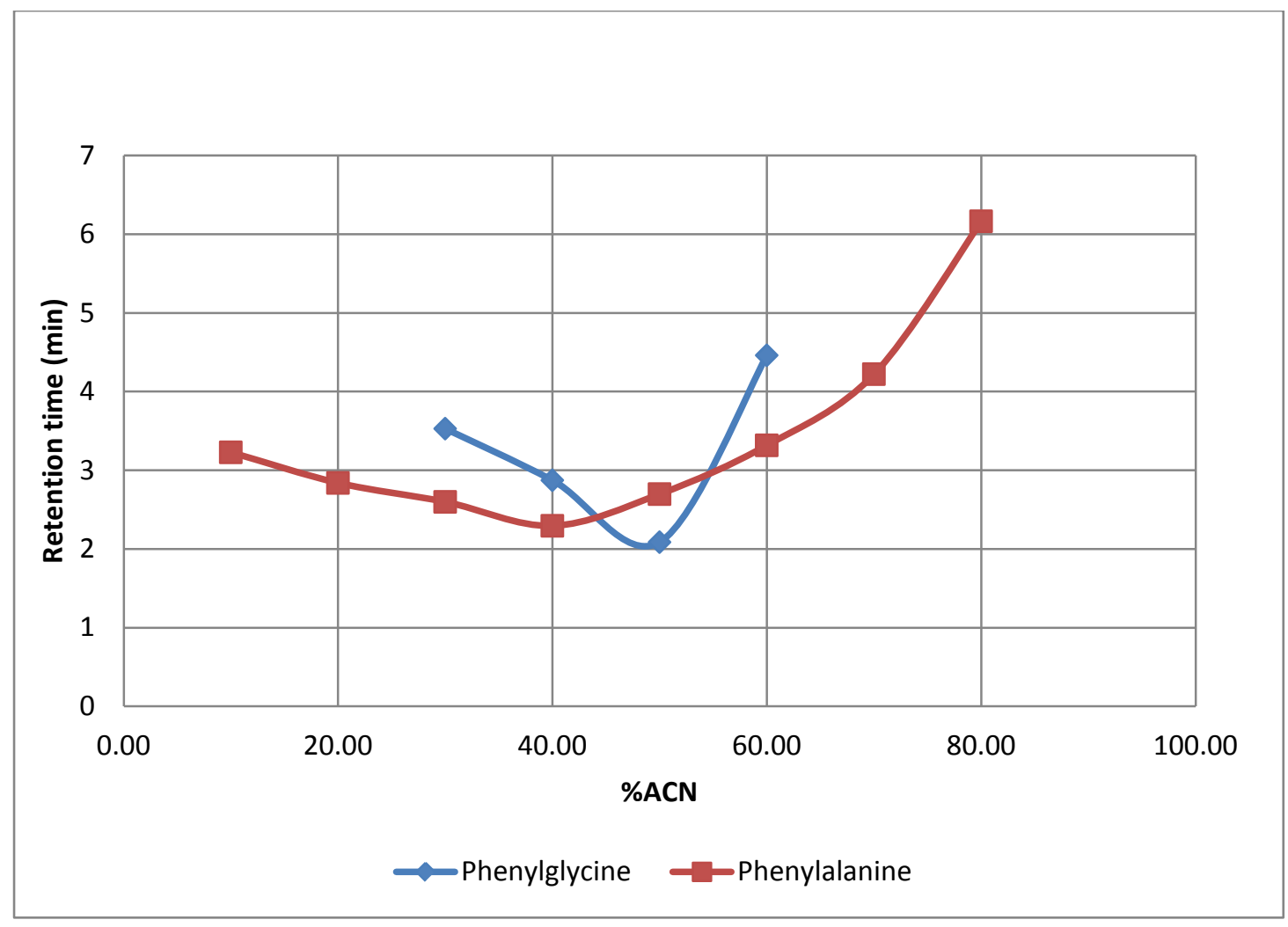

Figure 38. Retention map of the polar amino acids using the 6-chloro-1-hexyne column 
Table 25. Retention times of the hydrophilic nucleobases using the 6-chloro-1-hexyne column.

\begin{tabular}{|c|c|c|c|}
\hline \multicolumn{2}{|c|}{ Concentration of the mobile } & \multicolumn{2}{c|}{ Retention time } \\
phase (\%) & Adenine & Guanine \\
\hline Acetonitrile & DI water & $*$ & $*$ \\
\hline 10 & 90 & 13.7920 & 3.8220 \\
\hline 20 & 80 & 10.5290 & 3.2260 \\
\hline 30 & 70 & 9.1750 & 4.4430 \\
\hline 40 & 60 & 8.8420 & 5.3870 \\
\hline 50 & 50 & 9.3770 & 5.8320 \\
\hline 60 & 40 & 10.8570 & 6.0260 \\
\hline 70 & 30 & 13.8410 & \\
\hline 90 & 20 & $*$ & \\
\hline * peak was not observed & 10 & & \\
\hline
\end{tabular}

* peak was not observed 


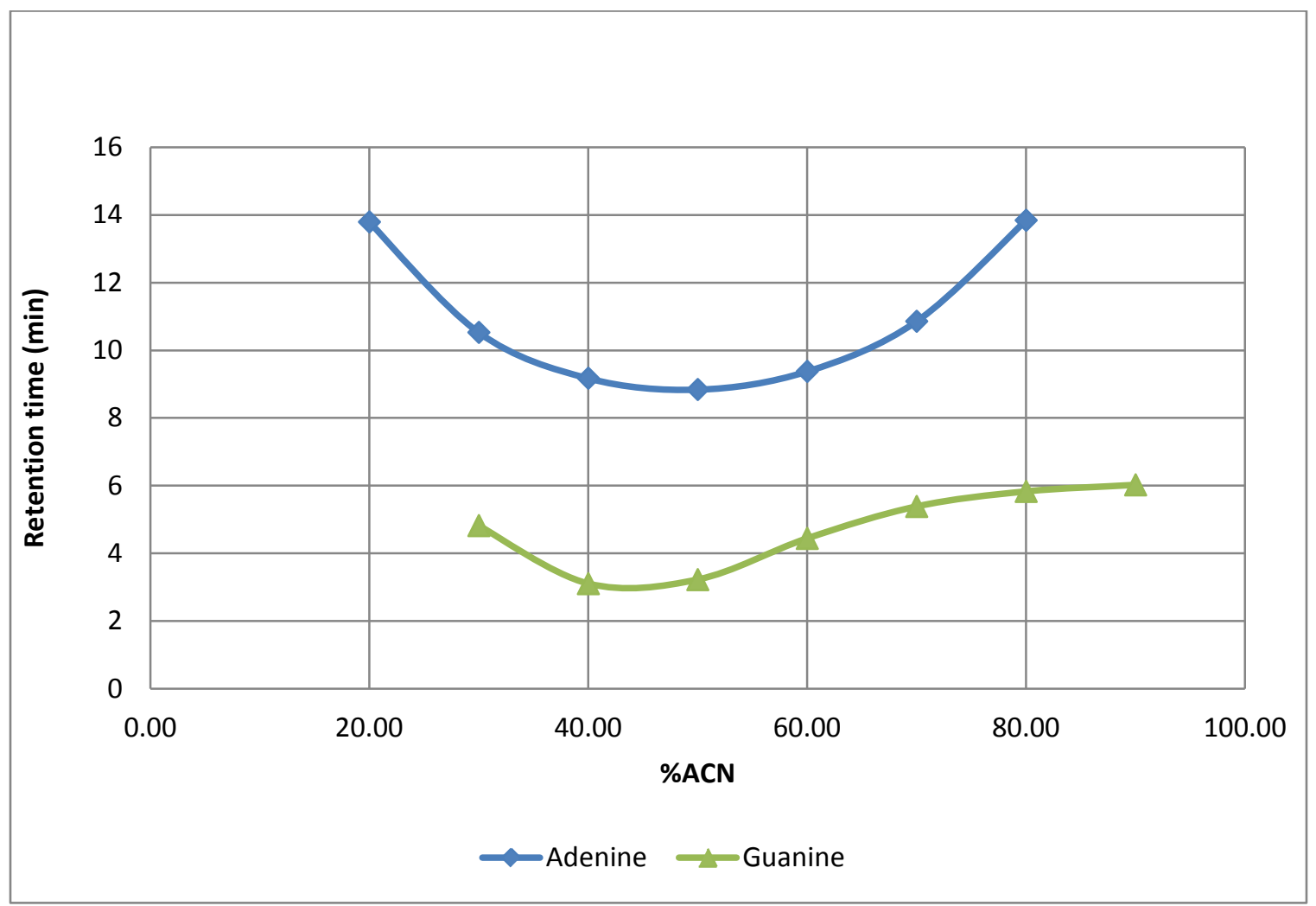

Figure 39. Retention map of the polar nucleobases using the 6-chloro-1-hexyne column 
Table 26. Retention times of the hydrophilic neurotransmitters using the 6-chloro-1hexyne column.

\begin{tabular}{|c|c|c|c|c|}
\hline \multicolumn{2}{|c|}{ Concentration of the } & \multicolumn{3}{c|}{ (min) } \\
mobile phase (\%) & & Norepinephrine & Dopamine \\
\hline Acetonitrile & DI water & Epinephrine & & 5.2470 \\
\hline 10 & 90 & 2.3250 & 3.0120 & 4.2020 \\
\hline 20 & 80 & 2.2150 & 2.5980 & 3.4050 \\
\hline 30 & 70 & 1.9990 & 2.3050 & 3.0700 \\
\hline 40 & 60 & 4.3670 & 2.0930 & 2.1360 \\
\hline 50 & 50 & 1.9960 & 3.5750 & 2.1620 \\
\hline 60 & 40 & 2.5110 & 5.3870 & 2.1740 \\
\hline 70 & 30 & 2.9960 & $*$ & $*$ \\
\hline 80 & 20 & 2.5300 & $*$ & $*$ \\
\hline 90 & 10 & $*$ & & \\
\hline
\end{tabular}

* peak was not observed

\section{ii. Hydrophobic analytes}

The non-polar compounds for this study when testing the 6-cholor-1-hexyne bonded stationary phase exhibit RP behavior entirely. The ANP behavior is somewhat limited, but molecules such as adrenosterone and corticosterone ( $\downarrow$ and $><$, respectively) in Figure 40 and naphthalene and pyrene ( and $\boldsymbol{\Delta}$, respectively) in Figure 41, exhibit both RP and ANP behavior. Tables 27 and 28 refer to the retention times for the two groups of hydrophobic molecules. Comparison of the retention times of hydrophilic and 
hydrophobic molecules, provided in Figure 42, shows that the 6-chloro-1-hexyne bonded silica hydride based stationary phase has reversed-phase as well as ANP properties.

Table 27. Retention times of the hydrophobic steroids using the 6-chloro-1-hexyne column.

\begin{tabular}{|c|c|c|c|c|c|c|}
\hline \multicolumn{2}{|c|}{$\begin{array}{c}\text { Concentration } \\
\text { of the mobile } \\
\text { phase }(\%)\end{array}$} & \multicolumn{5}{|c|}{$\begin{array}{c}\text { Retention time } \\
\text { (min) }\end{array}$} \\
\hline ACN & $\begin{array}{c}\text { DI } \\
\text { water }\end{array}$ & $\begin{array}{l}\text { Adreno } \\
\text { sterone }\end{array}$ & $\begin{array}{c}\Delta^{4} \text {-Androsten- } \\
\text { 3,17-dione }\end{array}$ & Esterone & Estradiol & Corticosterone \\
\hline 10 & 90 & $*$ & * & * & $*$ & * \\
\hline 20 & 80 & $*$ & $*$ & $*$ & 4.5490 & * \\
\hline 30 & 70 & 10.2600 & * & 4.3050 & 3.7490 & 7.8380 \\
\hline 40 & 60 & 5.7390 & 8.4580 & 4.0960 & 3.1260 & 4.3470 \\
\hline 50 & 50 & 3.8710 & 5.0420 & 3.2200 & 5.4000 & 3.0960 \\
\hline 60 & 40 & 4.2090 & 3.5230 & 2.5970 & 3.5730 & 2.5090 \\
\hline 70 & 30 & 5.3890 & 2.7690 & 2.2080 & $*$ & 2.2020 \\
\hline 80 & 20 & * & 2.3760 & 1.9860 & $*$ & 2.0530 \\
\hline 90 & 10 & * & * & * & * & 2.0130 \\
\hline
\end{tabular}

* peak was not observed 
Table 28. Retention times of the hydrophobic polycyclic aromatic hydrocarbons using the 6-chloro-1-hexyne column.

\begin{tabular}{|c|c|c|c|c|c|}
\hline \multicolumn{2}{|c|}{ Concentration of the } & \multicolumn{4}{|c|}{ (min) } \\
mobile phase (\%) & \multicolumn{5}{|c|}{ Phention time } \\
\hline ACN & DI water & Naphthalene & Phenthrene & Pyrene & Flourene \\
\hline 10 & 90 & $*$ & 3.2410 & 2.3260 & $*$ \\
\hline 20 & 80 & $*$ & 2.9430 & 2.1830 & 2.1990 \\
\hline 30 & 70 & $*$ & 2.7170 & 2.0010 & 11.5040 \\
\hline 40 & 60 & 9.0940 & 2.6560 & 3.0950 & 7.8340 \\
\hline 50 & 50 & 6.9830 & 2.8190 & 4.2570 & 6.9950 \\
\hline 60 & 40 & 10.3520 & 3.2440 & 5.0430 & 4.1640 \\
\hline 70 & 30 & $*$ & 3.8830 & 5.4050 & $*$ \\
\hline 80 & 20 & $*$ & 4.5280 & $*$ & $*$ \\
\hline 90 & 10 & $*$ & 5.3210 & $*$ & $*$ \\
\hline
\end{tabular}

* peak was not observed 


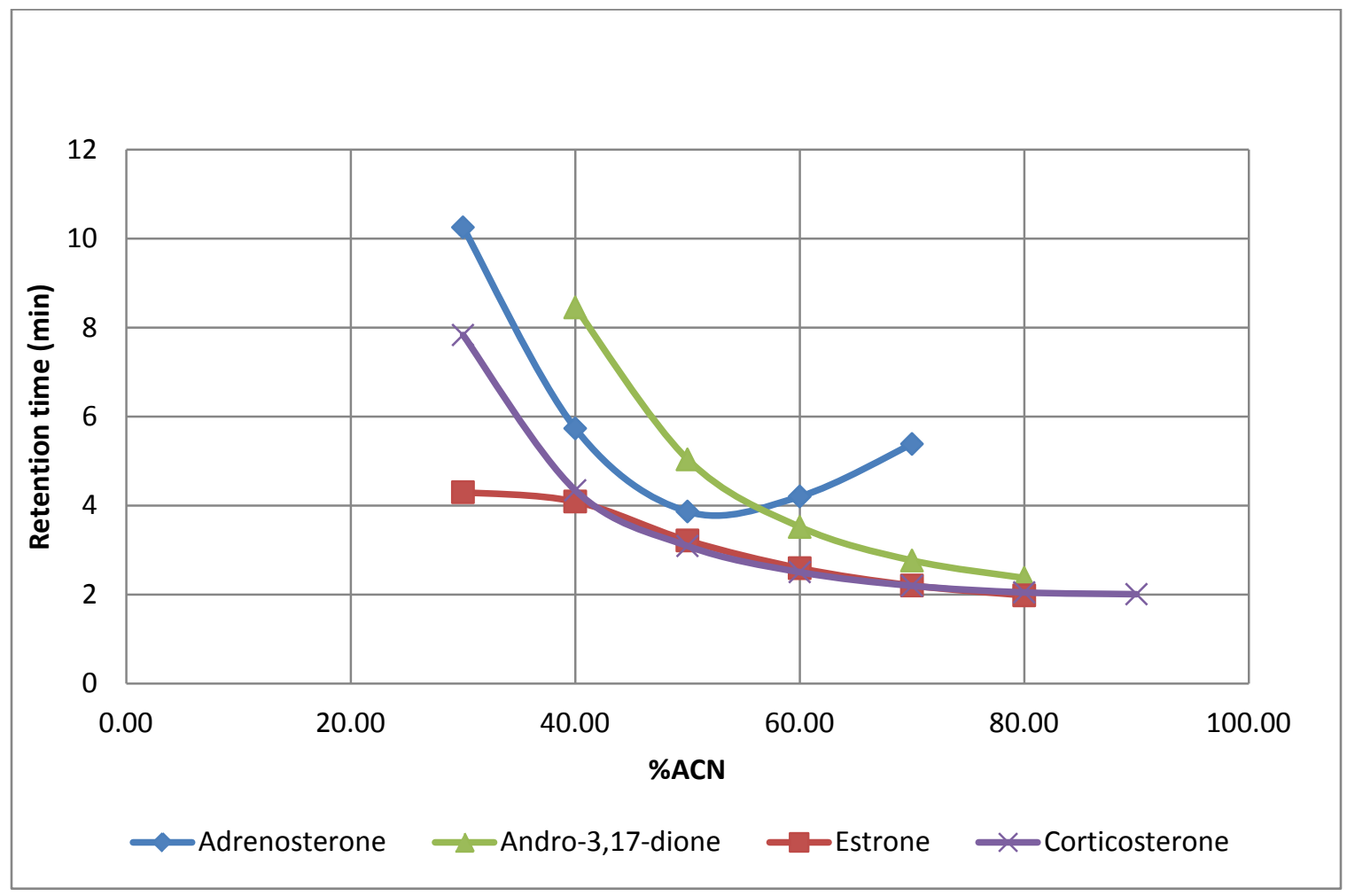

Figure 40. Retention map of the steroid analytes using the 6-chloro-1-hexyne column without buffer 


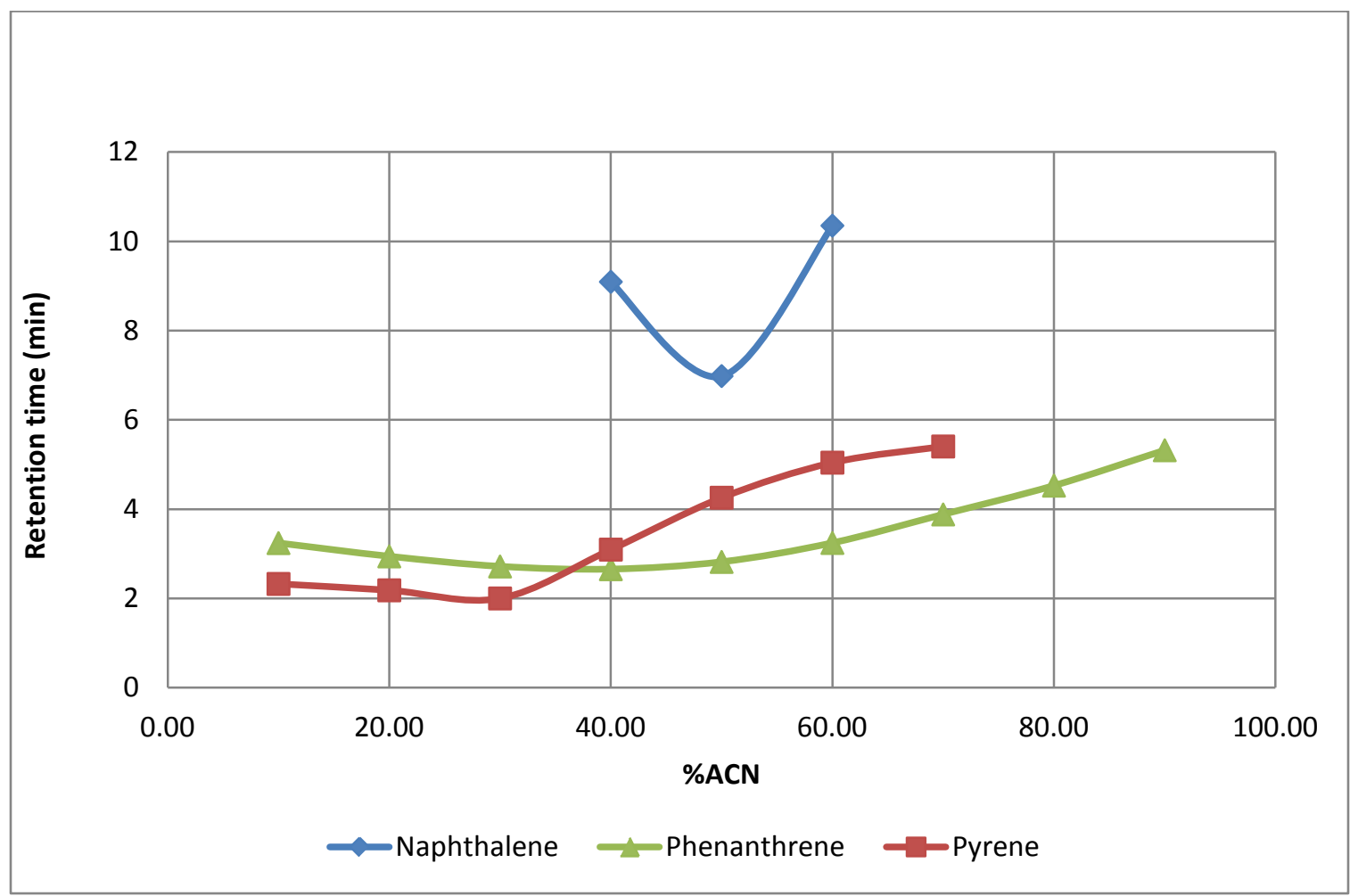

Figure 41. Retention map of the polycyclic aromatic hydrocarbon analytes using the 6chloro-1-hexyne column without buffer 


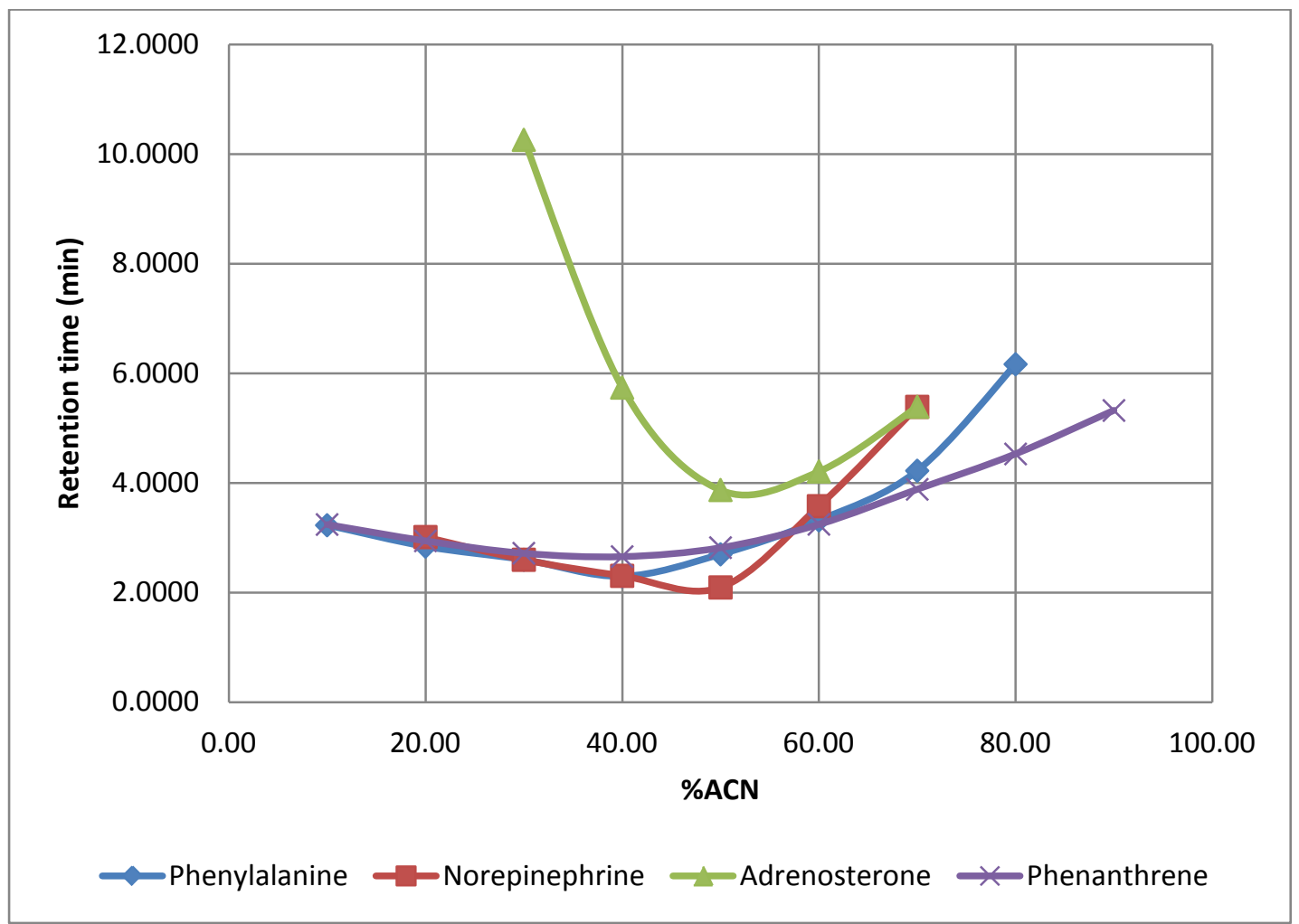

Figure 42. Retention map of the polar and non-polar analytes using the 6-chloro-1hexyne column without buffer

\section{4-Ethynylanaline}

\section{i. Hydrophilic analytes}

Three groups of hydrophilic (amino acids, nucleobases, and neurotransmitters) analytes were tested to evaluate RP/ ANP properties of 4-ethynylanaline. The retention times of these analytes for different polarities of the mobile phase are provided in Tables 29, 30, and 31. The results from the retention map plots for three groups of polar molecules in an isocratic mobile phase and 4-ethynylalanine stationary phase indicate that this column shows the RP phase properties and not so much of ANP, as shown in Figure 43. 
Table 29. Retention times of the hydrophilic amino acids using the 4-ethynylanaline column.

\begin{tabular}{|c|c|c|c|}
\hline \multicolumn{2}{|c|}{$\begin{array}{c}\text { Concentration of the mobile } \\
\text { phase (\%) }\end{array}$} & \multicolumn{2}{|c|}{$\begin{array}{l}\text { Retention time } \\
\text { (min) }\end{array}$} \\
\hline Acetonitrile & DI water & Phenylglycine & Phenylalanine \\
\hline 10 & 90 & $*$ & 8.2180 \\
\hline 20 & 80 & 17.6730 & 17.8270 \\
\hline 30 & 70 & 12.5730 & 12.6520 \\
\hline 40 & 60 & 9.9460 & 9.2320 \\
\hline 50 & 50 & 5.5610 & 7.4590 \\
\hline 60 & 40 & 6.2550 & 6.2660 \\
\hline 70 & 30 & 5.6090 & 5.6520 \\
\hline 80 & 20 & 4.9860 & 3.9230 \\
\hline 90 & 10 & 5.0360 & 4.9620 \\
\hline
\end{tabular}

* peak was not observed 
Table 30. Retention times of the hydrophilic nucleobases using the 4-ethynylanaline column.

\begin{tabular}{|c|c|c|c|}
\hline \multicolumn{2}{|c|}{ Concentration of the mobile } & \multicolumn{2}{c|}{ Retention time } \\
phase (\%) & Adenine & Guanine \\
\hline Acetonitrile & DI water & 24.6860 & 14.1820 \\
\hline 10 & 90 & 17.4390 & 10.0240 \\
\hline 20 & 80 & 14.5310 & 8.2360 \\
\hline 30 & 70 & 9.0570 & 5.9820 \\
\hline 40 & 60 & 7.0860 & 5.3310 \\
\hline 50 & 50 & 6.1530 & 4.6530 \\
\hline 60 & 40 & 5.7040 & 4.1050 \\
\hline 70 & 30 & 4.3550 & 4.0210 \\
\hline 90 & 20 & 4.4540 & \\
\hline
\end{tabular}


Table 31. Retention times of the hydrophilic neurotransmitters using the 4-ethynylanaline column.

\begin{tabular}{|c|c|c|c|c|}
\hline \multicolumn{2}{|c|}{ Concentration of the mobile } & \multicolumn{3}{c|}{ Retention time } \\
phase (\%) & & Norepinephrine & Dopamine \\
\hline Acetonitrile & DI water & Epinephrine & $*$ & 20.4870 \\
\hline 10 & 90 & $*$ & 17.2240 & 14.1930 \\
\hline 20 & 80 & 23.3980 & 12.4640 & 10.4360 \\
\hline 30 & 70 & 20.3290 & 9.0760 & 8.2570 \\
\hline 40 & 60 & 16.1980 & 7.5840 & 7.2750 \\
\hline 50 & 50 & 13.9630 & 6.1370 & 6.6470 \\
\hline 60 & 40 & 12.9630 & 5.8240 & 6.0730 \\
\hline 70 & 30 & 11.0330 & 5.2150 & 5.6460 \\
\hline 80 & 20 & 11.1130 & $*$ & $*$ \\
\hline 90 & 10 & $*$ & & \\
\hline & & & & \\
\hline
\end{tabular}




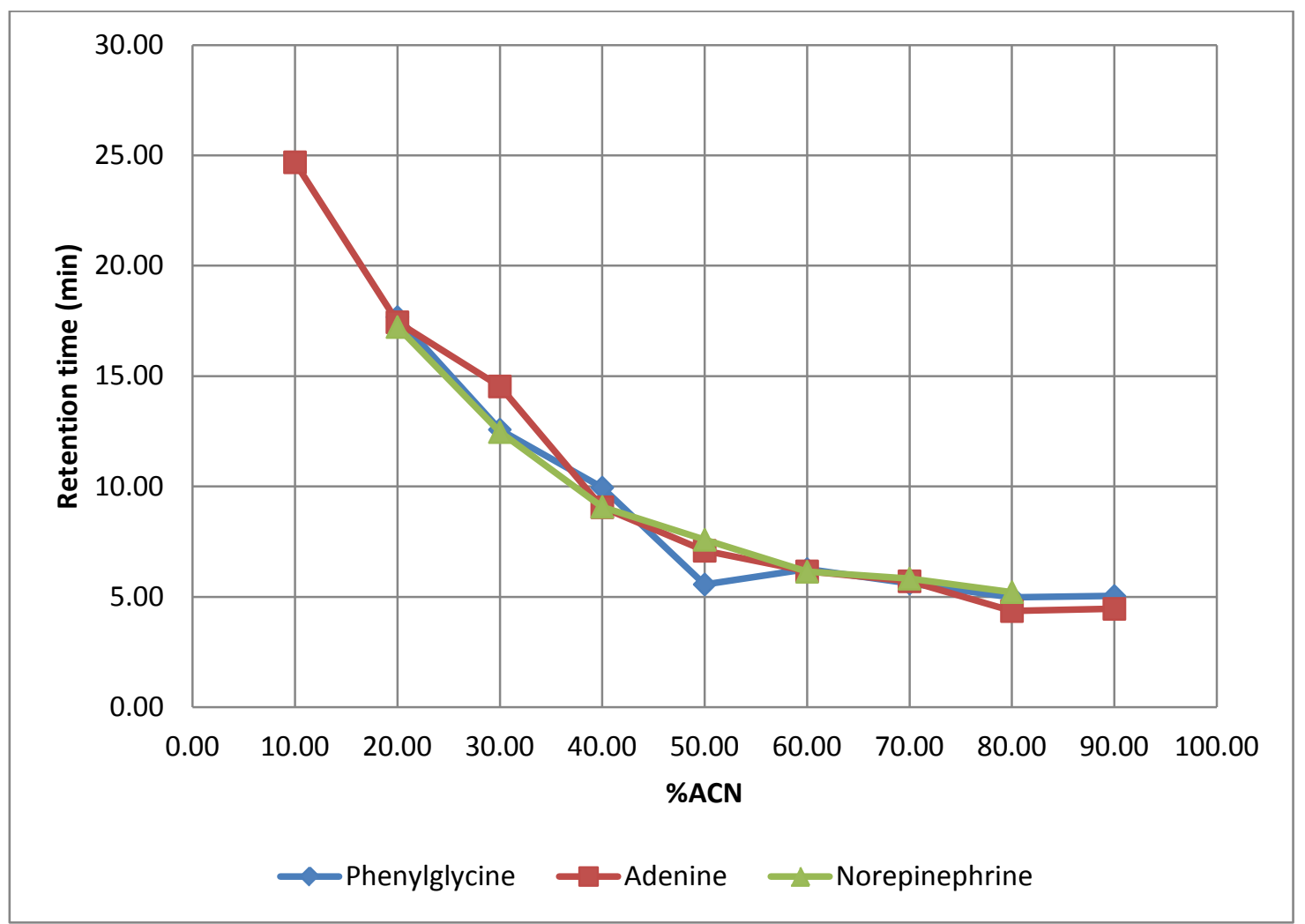

Figure 43. Retention map of the polar analytes using the 4-ethynylalanine column

\section{ii. Hydrophobic analytes}

The RP/ ANP behavior of a 4-ethynylalanine bonded stationary phase was tested using different molecules from two groups of hydrophobic molecules, steroids and polycyclic aromatic hydrocarbons. The retention times in different concentrations of ACN : $\mathrm{H}_{2} \mathrm{O}$ mobile phase are provided in Tables 32 and 33. 
Table 32. Retention times of the hydrophobic steroids using 4-ethynylanaline column.

\begin{tabular}{|c|c|c|c|c|c|c|}
\hline \multicolumn{2}{|c|}{$\begin{array}{c}\text { Conc. of the } \\
\text { mobile phase } \\
\text { (\%) }\end{array}$} & \multicolumn{5}{|c|}{$\begin{array}{c}\text { Retention time } \\
(\mathrm{min})\end{array}$} \\
\hline ACN & $\begin{array}{r}\text { DI } \\
\mathrm{H}_{2} \mathrm{O}\end{array}$ & $\begin{array}{l}\text { Adreno } \\
\text { sterone }\end{array}$ & \begin{tabular}{c}
\multicolumn{1}{c}{$\Delta^{4}$ - } \\
Androsten- \\
3,17-dione
\end{tabular} & Esterone & Estradiol & Corticosterone \\
\hline 10 & 90 & $*$ & * & $*$ & * & $*$ \\
\hline 20 & 80 & 17.4190 & 18.1060 & 20.0470 & 19.7910 & 15.8490 \\
\hline 30 & 70 & 12.6140 & 13.2760 & 14.8860 & 14.7480 & 14.4220 \\
\hline 40 & 60 & 9.4010 & 9.8430 & 10.9340 & 10.8530 & 10.7150 \\
\hline 50 & 50 & 7.4530 & 7.8880 & 8.7680 & 8.7550 & 8.7460 \\
\hline 60 & 40 & 6.2760 & 6.4360 & 7.4520 & 7.3370 & 7.3320 \\
\hline 70 & 30 & 5.7600 & 5.7970 & 6.6780 & 6.6730 & 6.5590 \\
\hline 80 & 20 & 5.3740 & 5.5130 & 6.2350 & 6.1730 & 6.1680 \\
\hline 90 & 10 & 4.6700 & $*$ & 6.6290 & 6.0040 & 6.4210 \\
\hline
\end{tabular}

* peak was not observed 
Table 33. Retention times of the hydrophobic polycyclic aromatic hydrocarbons using the 4-ethynylanaline column.

\begin{tabular}{|c|c|c|c|c|c|}
\hline \multicolumn{3}{|c|}{ Concentration of the } & \multicolumn{4}{|c|}{ Retention time } \\
mobile phase (\%) & & Phin) \\
\hline ACN & DI water & Naphthalene & Phenanthrene & Pyrene & Flourene \\
\hline 10 & 90 & $*$ & $*$ & 24.1050 & $*$ \\
\hline 20 & 80 & 17.4290 & 18.2920 & 17.0110 & 18.6090 \\
\hline 30 & 70 & 12.3010 & 15.3710 & 12.0310 & 11.2680 \\
\hline 40 & 60 & 9.1690 & 9.6610 & 8.9960 & 9.6340 \\
\hline 50 & 50 & 7.6760 & 7.9790 & 7.2770 & 7.8380 \\
\hline 60 & 40 & 6.1740 & 6.4990 & 6.0410 & 6.6920 \\
\hline 70 & 30 & 5.5440 & 5.9240 & 5.4180 & 6.1060 \\
\hline 80 & 20 & 5.2580 & 5.6650 & 5.1460 & 5.7670 \\
\hline 90 & 10 & 4.8330 & 5.0580 & 4.6830 & 5.3080 \\
\hline
\end{tabular}

* peak was not observed 


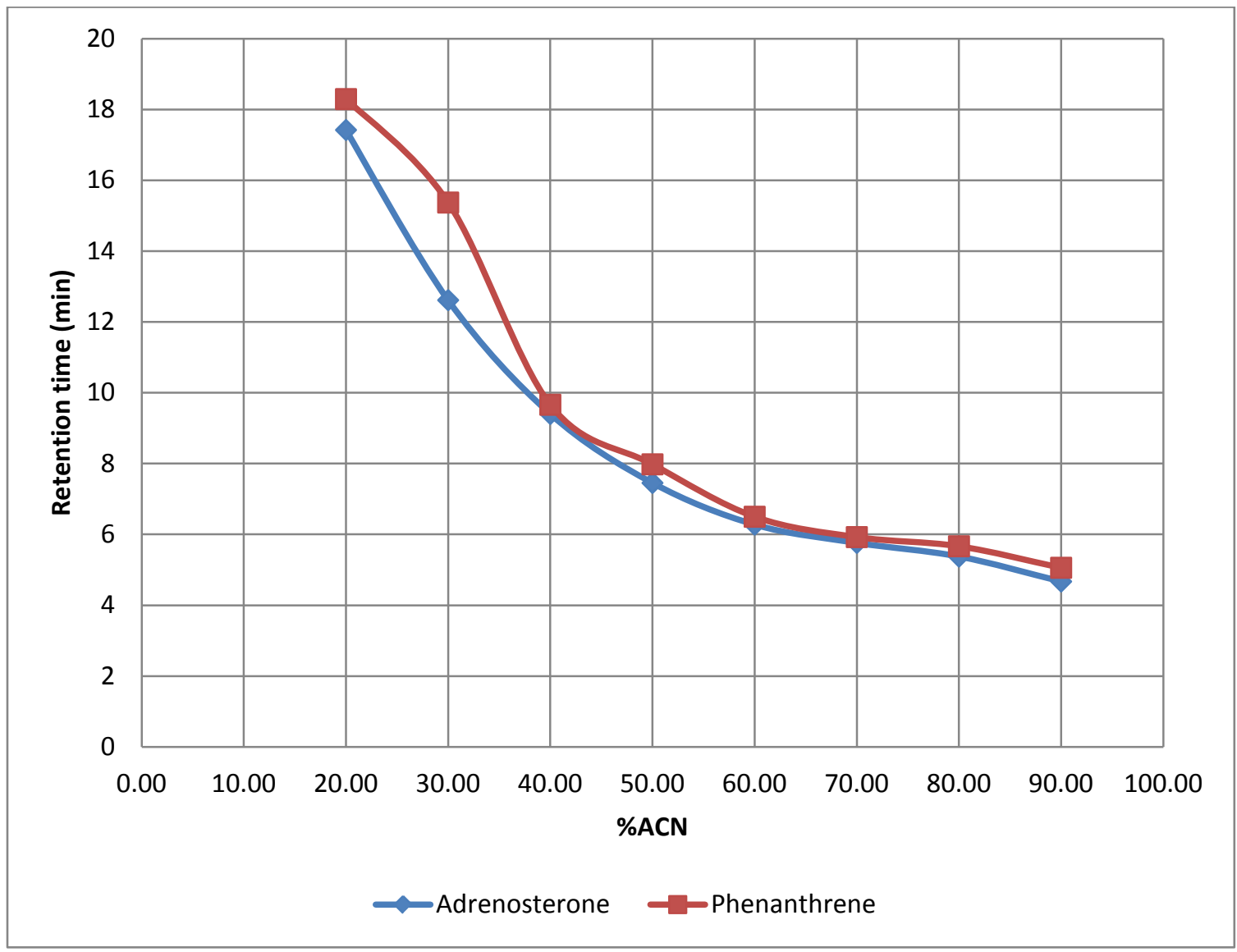

Figure 44. Retention map of the hydrophobic analytes using the 4-ethynylalanine column

Using one of the analytes from each group, both adrenosterone (a steroid) and phenanthrene (a polycyclic aromatic hydrocarbon) show similar results when run on the 4-ethynylanaline column. As shown in Figure 44, adrenosterone and phenanthrene ( and $\mathbf{\square}$, respectively) exhibit RP behavior only. 


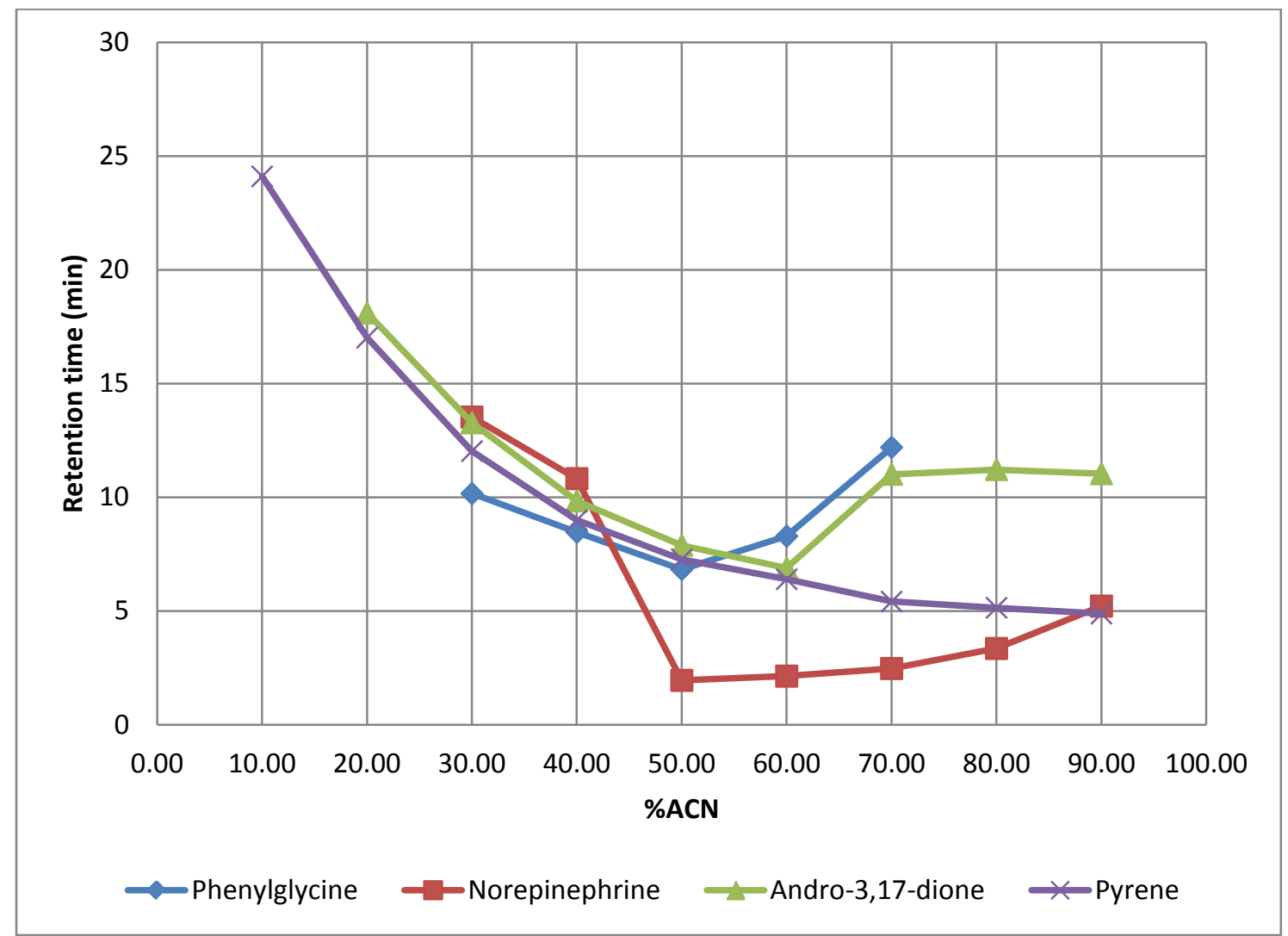

Figure 45. Retention map of the polar and non-polar analytes using the 4-ethynylanaline column without buffer

Figure 45 shows the retention maps for phenylglycine (polar), norepinephrine (polar), 4-androstene-3,17-dione (slightly non-polar) and pyrene (non-polar) using the 4ethynylalanine column, ( $\bullet, \mathbf{\square}, \boldsymbol{\Delta}$, and $><$, respectively). A comparison of the analyte retention maps shows that the stationary phase 4- ethynylalanine has reversed-phase properties but does not possess significant ANP properties. 


\section{CONCLUSIONS}

The two foci of this study were to synthesize novel stationary phases that could perform in both reversed-phase and aqueous normal phase modes and characterization and identification of each column, in terms of the RP/ANP properties. Four organic moieties were successfully bonded to a silica hydride surface via silanization/ hydrosilation methods. Carbon analysis, DRIFT, and solid-state NMR all confirmed the attachment of four (out of five) organic moieties to the surface of the silica hydride base. HPLC tests on each column with a wide range of hydrophilic and hydrophobic analytes and isocratic conditions of $\mathrm{ACN}: \mathrm{H}_{2} \mathrm{O}$ mobile phases were designed to evaluate the RP, ANP, or RP/ANP mode of each newly synthesized column. It appeared that under these conditions, the 1,8-nonadiyne and 6-chloro-1-hexyne-bonded stationary phases showed both RP and ANP behaviors. The 5-cyano-1-pentyne-bonded stationary phase exhibited only ANP behavior, whereas the 4-ethynylanaline functioned only in the reversed-phase mode. Furthermore, the reproducibility studies and \%RSD values of the columns, the 1,8-nonadiyne-bonded stationary phase in particular, confirmed the high degree of precision in obtaining the retention data.

However, the effect of other variables that were not factored in to this study and that might have shifted the results favorably or unfavorably should not be ignored. For instance, when performing the chromatographic runs with 5-cyano-1-pentyne-bonded stationary phase, there was the appearance of noise across the chromatogram for $\leq 30 \%$ ACN: $\mathrm{H}_{2} \mathrm{O}$ concentrations of the binary mobile phase. 
The study also confirmed that the presence of a buffer (formic acid or other acidic additives) in the mobile phase played a significant role in the appearance or disappearance of RP, ANP, or RP/ANP behavior. The presence of $0.1 \%(\mathrm{v} / \mathrm{v})$ formic acid in the binary mobile phase caused an enhancement in the ANP behavior of columns that exhibited this property. In the case of the 5-cyano-1-hexyne-bonded stationary phase, adding buffer ( $0.1 \%$ volumetric) to the binary mobile phase caused the disappearance of noise in the $10 \%-30 \%$ ACN region, allowing the bonded stationary phase to exhibit RP behavior.

The surface coverage of the bonded phase also played a role in the retention of analytes. The higher the surface coverage, the more likely for the analyte to be retained on the stationary phase, hence producing a longer retention time.

Additional work could be done to consider the effect of temperature on the RP/ANP behavior of stationary phases. Investigating the effect of various buffers could also provide valuable information on the retention properties, since in this study the ANP behavior of stationary phases was enhanced due to the presence of a small amount of acidic buffer in the mobile phase. Expanding the range of the analytes to molecules such as sugars and proteins/ peptides could provide additional information on behavior of newly synthesized stationary phases. 


\section{REFERENCES}

1. Skoog, A. D.; Holler, F. J.; Nieman, T. A. Principles of Instrumental Analysis, 5th ed., Saunders College Publishing: Orlando, Florida, 1998, Chapters 26, 28.

2. Vladimar, B. Journal of Chromatography A. 1989, 477, 187-233.

3. Parris, N. A., Instrumental Liquid Chromatography, Journal of Chromatography Library, Elsevier B.V., 1976, 5, iii-x.

4. Weston, A.; Brown, P. Introduction to High Performance Liquid

Chromatography, Chapman \& Hall, London, NY, 1982, Chapter 1-6.

5. The Chemguide homepage. http ://www.chemguide.co.uk/analysis/

chromatography/ hplc.html. (accessed February $1^{\text {st }}, 2012$ ).

6. Cunico, R. L.; Gooding, K. M.; Wehr, T. Basic HPLC and CE of Biomolecules, Bay Bioanalytical Laboratory, CA, 1998.

7. Lendi, B. E.; Meyer, V. R. LC-GC Europe, 2005, 18, 3, 156-163.

8. Scott, R. P. W. Principles and Practice of Chromatography, Chrom-Ed Book Series, Library for Science, 2003, 65.

9. Pesek, J. J.; Matyska, M. T. LCGC North America. 2006, 24, 3, 296-303.

10. Pesek, J. J.; Matyska, M. T.; Fischer, S. M.; Sana, T. R. J. Chromatogr. A. 2008, 1204, 48-55.

11. Hemstrom, P.; Irgum, K. J. Sep. Sci. 2006, 29, 1784-1821.

12. Dorsey, J. G.; Cooper, W. T. Anal. Chem. 1994, 66, 857A-867A. 
13. Vansant, E. F.; Van Der Voort, P.; Vrancken, K. C. Characterization and Chemical Modification of Silica; Elsevier: Amsterdam, New York, 1995, Chapter 1-6.

14. Pesek, J. J.; Matyska, M. T. J. Sep. Sci. 2005, 28, 1845-1854.

15. Jacek, N. J. Chromatogr A. 1997, 779, 1, 29-71.

16. Pesek, J. J; Matyska, M. T.; Takhar, S. Chromatographia, 1998, 48, 631-636.

17. Pesek, J. J.; Matyska, M. T.; Sandoval, J. E.; Williamsen, E. J. J. Liq. Chrom. \& Rel. Technol. 1996, 19. 2843-3865.

18. Pesek, J. J.; Matyska, M. T. Interface Sci. 1997, 5, 103-117.

19. Pesek, J. J.; Matyska, M. T. J. Chromatogr. A 2005, 1079, 366-371.

20. Chu, C. H.; Jonsson, E.; Auvinen, M.; Pesek, J. J.; Sandoval, J. E. Anal. Chem. 1993, 65, 808-816.

21. Gee, M. L.; Healy, T. W.; White, L. R. J. Colloid Interface Sci. 1990, 140, 450465.

22. Gomez, J. E.; Sandoval, J. E. Anal. Chem. 2010, 82, 7444-7451.

23. Sandoval, J. E.; Pesek. J. J. Anal. Chem. 1989, 61, 2067-2075.

24. Pesek, J. J.; Matyska, M. T.; Muley, S. Chromatographia 2000, 52, 439-444.

25. Pesek, J. J.; Matyska, M. T.; Olivia, M.; Evanchic, M. J. Chromatogr. A. 1998, 818, 145-154. 
26. Akapo, S. O.; Dimandja, J.-M. D.; Pesek, J. J.; Matyska, M. T. Chromatographia 1996, 42, 141-146.

27. Pesek, J. J.; Matyska, M. T.; Suryadevara, R. J. Liq. Chrom. \& Rel. Technol. 2005, 28, 2111-2139.

28. Silverstein, R. M.; Bassler, G. L.; Morril, T. C. Spectroscopic Identification of Organic Compounds, $4^{\text {th }}$ ed., John Wiley \& Sons, New York, NY, 1963, Chapter $1-6$.

29. Berendsen, G. E.; De Galan, L. H. J. Liq. Chromatogr. 1978, 1, 561-569.

30. Babonneau, F.; Baccile, N.; Laurent, G.; Maquet, J.; Azaïs, T.; Gervais, C.; Bohnomme, C. C. R. Chimie 2010, 13, 58-68.

31. Pesek, J. J.; Matyska, M. T. Adsorption and its Applications in Industry and Environmental Protection, Studies in Surface Science and Catalysis A. Dabrowski, 1998, 117-142.

32. Babonneau, F.; Maquet, J., Polyhedron, 2000, 19, 315-322.

33. Glase, R. H.; Wilkes, G. L.; Bronnimann, C. E. J. Non-Cryst. Solids, 1989, 113, 73.

34. The Windsor homepage. http://mutuslab.cs.uwindosor.ca./schurko/ssnmr/ ssnmr_schurko. pdf. (accessed February $7^{\text {th }}, 2012$ )

35. The GMI homepage. http://www.gmi-inc.com/HP-1090-Series-HPLCsystem.html (accessed February $7^{\text {th }}$, 2012).

36. The Grace homepage. http://www.discoverysciences.com/product.aspx?id=2604 (accessed February $7^{\text {th }}, 2012$ ). 